\title{
The immunoregulatory landscape of human tuberculosis granulomas
}

\author{
Erin F. McCaffrey $\mathbb{B}^{1}$, Michele Donato ${ }^{2,3}$, Leeat Keren ${ }^{4}$, Zhenghao Chen ${ }^{5}$, Alea Delmastro ${ }^{1}$,

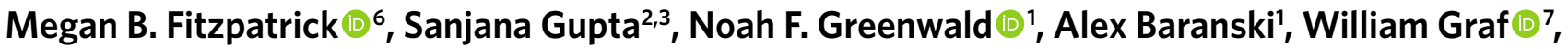 \\ Rashmi Kumar1, Marc Bosse', Christine Camacho Fullaway ${ }^{1}{ }^{1}$, Pratista K. Ramdial ${ }^{8}$, Erna Forgó1, \\ Vladimir Jojic ${ }^{5}$, David Van Valen ${ }^{7}{ }^{7}$, Smriti Mehra9 , Shabaana A. Khader ${ }^{10}{ }^{10}$, Sean C. Bendall (1) \\ Matt van de Rijn', Daniel Kalman"1, Deepak Kaushal ${ }^{12}$, Robert L. Hunter ${ }^{13}$, Niaz Banaei ${ }^{1,14}$, \\ Adrie J. C. Steyn ${ }^{8,15}$, Purvesh Khatri ${ }^{2,3}$ and Michael Angelo (iD) 1 讹
}

Tuberculosis (TB) in humans is characterized by formation of immune-rich granulomas in infected tissues, the architecture and composition of which are thought to affect disease outcome. However, our understanding of the spatial relationships that control human granulomas is limited. Here, we used multiplexed ion beam imaging by time of flight (MIBI-TOF) to image 37 proteins in tissues from patients with active TB. We constructed a comprehensive atlas that maps 19 cell subsets across 8 spatial microenvironments. This atlas shows an IFN- $\gamma$-depleted microenvironment enriched for TGF- $\beta$, regulatory T cells and IDO1 ${ }^{+}$ PD-L1+ myeloid cells. In a further transcriptomic meta-analysis of peripheral blood from patients with TB, immunoregulatory trends mirror those identified by granuloma imaging. Notably, PD-L1 expression is associated with progression to active TB and treatment response. These data indicate that in TB granulomas, there are local spatially coordinated immunoregulatory programs with systemic manifestations that define active TB.

M ycobacterium tuberculosis $(M t b)$ infection accounts for nearly 1.5 million deaths each year ${ }^{1}$, prompting efforts to develop new host-directed therapies to treat TB disease. However, these efforts have been hindered by an incomplete understanding of how the human immune system responds to $M t b$. Infection is initiated when bacteria are engulfed by phagocytic cells after being inhaled into the lungs ${ }^{2,3}$. This triggers an immune response that converges on formation of a granuloma, a dynamic and spatially organized tissue structure composed of macrophages, granulocytes, lymphocytes and fibroblasts. From the perspective of facilitating an effective host response, granulomas play contradictory roles. On one hand, consolidation of infected cells within the myeloid core limits dissemination by partitioning them away from uninvolved lung parenchyma. On the other, tolerogenic pathways upregulated within this region may limit bacterial clearance ${ }^{4-6}$.

Granuloma composition can be highly variable ${ }^{7}$. Even within a single individual, infection can result in granulomas with distinct histologic features that each progress independently over time $^{8}$. Controlled infections in non-human primates have revealed that a single individual can possess well over ten granulomas, and the inflammatory profile, size and bacterial ecology of these lesions differ dramatically ${ }^{9-11}$. Thus, the trajectory of each granuloma varies across a spectrum between complete bacterial clearance to uncontrolled dissemination. This discordance suggests that local host-bacterial dynamics within the tissue microenvironment (ME) play a central role in determining granuloma fate. Along these lines, a growing number of studies find that granuloma structure and immune cell function are interconnected ${ }^{12-15}$

Taken together, these findings suggest that TB progression is impacted by focal, spatially encoded regulatory mechanisms within the granuloma ME. Thus, understanding how these mechanisms promote bacterial clearance or persistence is critical for designing effective therapies. A necessary first step toward this goal is to characterize immune cell dynamics and regulatory pathways in human TB granulomas. However, many facets of TB granuloma pathology are human specific and difficult to emulate in model systems. This is compounded by the fact that, unlike other tissue pathologies, biopsy specimens are rarely obtained during a typical TB clinical workup. In this respect, TB is a member of a larger class of diseases, where the paucity of human material and lack of high-fidelity model systems have impeded development of new therapies.

With this in mind, we applied a stepwise investigative framework where limited amounts of archival tissue and publicly available transcriptome data are integrated to glean new insight into human-specific pathobiology in TB. We employed MIBI-TOF ${ }^{16}$ to

'Department of Pathology, Stanford University School of Medicine, Stanford, CA, USA. ${ }^{2}$ Department of Medicine, Division of Biomedical Informatics Research, Stanford University School of Medicine, Stanford, CA, USA. ${ }^{3}$ Institute for Immunity, Transplantation and Infection, Stanford University School of Medicine, Stanford, CA, USA. ${ }^{4}$ Department of Molecular Cell Biology, Weizmann Institute of Science, Rehovot, Israel. ${ }^{5}$ Calico Life Sciences LLC, South San Francisco, CA, USA. ${ }^{6}$ Department of Pathology, University of Wisconsin, Madison, WI, USA. 'Division of Biology and Bioengineering, California Institute of Technology, Pasadena, CA, USA. ${ }^{8}$ Africa Health Research Institute, University of KwaZulu-Natal, Durban, South Africa. ${ }^{9}$ Texas Biomedical Research Institute, San Antonio, TX, USA. ${ }^{10}$ Department of Molecular Microbiology, Washington University School of Medicine, St. Louis, MO, USA. "Department of Pathology and Laboratory Medicine, Emory University School of Medicine, Atlanta, GA, USA. ${ }^{12}$ Southwest National Primate Research Center, Texas Biomedical Research Institute, San Antonio, TX, USA. ${ }^{13}$ Department of Pathology and Laboratory Medicine, University of Texas Health Sciences Center at Houston, Houston, TX, USA. ${ }^{14}$ Division of Infectious Diseases \& Geographic Medicine, Department of Medicine, Stanford University, Stanford, CA, USA.

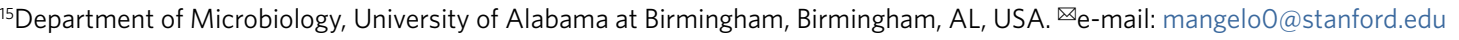


chart granuloma composition across eight computationally defined spatial MEs, revealing features of highly localized immune modulation in granulomas, such as IDO1- and PD-L1-expressing myeloid cells, proliferative regulatory $\mathrm{T}$ cells $\left(\mathrm{T}_{\text {reg }}\right.$ cells) and high levels of transforming growth factor $\beta$ (TGF- $\beta$ ) alongside depletion of IFN- $\gamma$. We find that IDO1 seems to be specific to TB granulomas, whereas PD-L1 and sparsity of activated T cells are also found in another granulomatous condition, sarcoidosis. Lastly, in an orthogonal analysis of blood transcriptomes from patients with TB, we observe that similar immunoregulatory expression dynamics define systemic immunity during active TB.

\section{Results}

Structured immune cell composition in human TB granulomas. To assess granuloma composition and architecture in TB, we curated a cohort of actively infected human tissues. Archival formalin-fixed paraffin-embedded (FFPE) specimens from patients treated in the United States or South Africa were procured from Stanford Health Care and University of Texas Health Science Center or University of KwaZulu-Natal, Inkosi Albert Luthuli Central Hospital, respectively (Extended Data Table 1). The South African cohort comprised pulmonary tissues from patients undergoing therapeutic resection for advanced TB $(n=3)$, whereas a subset of US specimens came from postmortem autopsy lung tissues from patients with fatal TB $(n=3)$. Although TB disease typically manifests in the lung, infection can disseminate to extrapulmonary sites ${ }^{17}$. To characterize $\mathrm{TB}$ infection at an earlier stage and assess how granuloma composition varies with infection site, we included diagnostic biopsy specimens from lung $(n=2)$, pleural cavity $(n=3)$, lymph node $(n=2)$, vertebrae $(n=1)$ and endometrium $(n=1)$ (Fig. 1a).

Each specimen was reviewed by an anatomic pathologist and screened to include the presence of active granulomatous inflammation (Extended Data Fig. 1a). MIBI-TOF was subsequently used to image two $500 \mu \mathrm{m} \times 500 \mu \mathrm{m}$ fields of view (FOVs) per tissue after staining with a 37-plex panel of metal-labeled antibodies (Fig. 1b, Extended Data Fig. 1b,c and Extended Data Table 2) (ref. ${ }^{16}$ ). The antibody panel included markers to phenotype major immune and nonimmune cell lineages, including lymphocytes, macrophages, granulocytes, stroma and epithelium. The panel also included antibodies for 12 functional markers, including those with well-documented immunoregulatory activity, such as PD-1, Lag3, PD-L1 and IDO1.

To extract single cells, multiplexed imaging data were processed with a low-level pipeline prior to single-cell segmentation (Fig. 1a and Extended Data Fig. 1d $)^{18-20}$. Each FOV contained an average of $\sim 1,410$ single cells (s.d.=343) (Extended Data Fig. 2 d). FlowSOM (Extended Data Fig. 2a,b) was employed to phenotype 19 unique cell subsets (Fig. 1c) (ref. ${ }^{21}$ ). For each image, FlowSOM clusters and segmentation masks were combined to generate cell phenotype maps (CPMs), where each cell is labeled by its phenotype (Fig. 1d and Extended Data Fig. 2c).
Granuloma composition was predominated in most lesions by $\mathrm{T}$ cells and myeloid cells, (average myeloid/lymphoid ratio $=2.4$, s.d. = 2.4). Myelomonocytic cells comprised multiple subsets of macrophages, dendritic cells (DCs) and monocytes that were distinguished by varying degrees of coexpression of CD11c, CD11b, CD209, CD68, CD14, CD16 and CD206 (Fig. 1c,e). Granulocytes consisted of neutrophils (mean $=2.5 \%$, s.d. $=8.8 \%$, of total immune cells) and mast cells $(0.6 \% \pm 0.9)$. We also identified $\gamma \delta$ T cells $(0.1 \%$ $\pm 0.3)$, CD209+ DCs $(0.2 \% \pm 0.6)$ and $\mathrm{T}_{\text {reg }}$ cells $(1.0 \% \pm 1.7)$, highlighting the capability of our approach to enumerate low-abundance cell populations that are suggested to play a key role in granuloma pathology. In line with increased vascularization in active disease $^{22,23}$, nonimmune cells were predominated in most lesions by endothelial cells $(3.7 \% \pm 2.8$, of total cells), whereas fibroblasts $(5.6 \% \pm 6.5)$ and epithelial cells $(2.0 \% \pm 3.7)$ varied between lesions (Extended Data Fig. 2e,f). Altogether, we assigned 94\% $(n=39,709$ single cells) of cells to 19 subsets that ranged in frequency from $0.1 \%$ to $15 \%$ across our dataset.

To understand the relationship between granuloma composition and organ site, we compared cell abundances between pulmonary and extrapulmonary tissues. Interestingly, we found the vast majority of subsets (13/19) occurred in similar proportions irrespective of organ site (Extended Data Fig. 2g,h). However, pulmonary tissues displayed increased proportions of mast cells $(P=0.002$, Wilcoxon rank sum), $\mathrm{CD}^{+} 8^{+}$macrophages $(P=0.007)$, and multinucleated giant cells (MNGCs; $P=0.03$ ), along with a slight decrease in

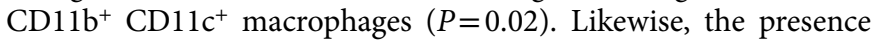
of $\mathrm{CD} 14^{+} \mathrm{CD} 16^{+}$intermediate monocytes was nearly exclusive to extrapulmonary tissues $\left(P=6 \times 10^{-5}\right)$.

Given the subtle differences across organ sites, we next evaluated how granuloma composition varied with clinical origin. For this, we compared diagnostic biopsy specimens with advanced disease in postmortem and resection tissues (Fig. 1e-h and Extended Data Fig. 2i,j). First, we found differences in $\mathrm{CD} 8^{+} \mathrm{T}$ cell frequency drove skewing of the $\mathrm{CD}^{+}$to $\mathrm{CD}^{+} \mathrm{T}$ cell ratio between groups, with postmortem and resection tissues exhibiting the lowest and highest proportion of $\mathrm{CD} 8^{+} \mathrm{T}$ cells, respectively $(P=0.005$, Wilcoxon rank sum). Second, therapeutic resections were preferentially depleted of $\mathrm{CD}_{11} \mathrm{~b}^{+} \mathrm{CD} 11 \mathrm{c}^{+}$macrophages and instead enriched for $\mathrm{CD} 14^{+}$monocytes. Notably, we found these two trends were moderately correlated $\left(R^{2}=0.18, r=0.43\right.$ and $P=0.026, t$ test; Fig. 1i). Although both sample types were from patients with advanced disease, the clinical course of patients undergoing resection differed from that of postmortem specimens due to the acute, presurgical antimicrobial treatment the patient had received. Thus, it is possible that coordinated $\mathrm{CD}^{+} \mathrm{T}$ cell and monocyte recruitment is driven by presurgical antimicrobial therapy, although other differences between these specimens should be considered. Altogether, this comprehensive cell census revealed distinct types of granulomas that are defined by immune cell frequency and associate with TB disease status.

Fig. 1 | Multiplexed imaging of TB granulomas reveals structured immune cell composition. a, Conceptual overview of MIBI-TOF analysis of human TB granulomas, comparison with sarcoidosis and complementary analysis of systemic responses to TB. $\mathbf{b}$, Representative images from a TB granuloma. c, Cell lineage assignments based on normalized expression of lineage markers (heatmap columns). Rows are ordered by absolute abundance shown on the bar plot (left), whereas columns are hierarchically clustered (Euclidean distance, average linkage). d, Cell identity overlaid onto the segmentation mask for a representative TB granuloma (left). Two insets (right) are shown. e, The relative abundance of immune cell types across all TB FOVs with cell types ordered by decreasing median abundance and bars ordered by specimen origin (resection, blue; postmortem, green; diagnostic biopsy, red). f, Frequency of $\mathrm{CD} 14^{+}$monocytes and $11 \mathrm{~b} / \mathrm{c}^{+} 206^{+}$macrophages among total immune cells colored by specimen origin. Line represents the median. $\mathbf{g}$, The CD4+ $\mathrm{T}$ cell/CD8 ${ }^{+} \mathrm{T}$ cell ratio represented as a $\log _{2}$ fold change for each TB FOV (top) colored by specimen origin (top) and frequency of CD4+ $\mathrm{T}$ cells (middle) and CD8 ${ }^{+} \mathrm{T}$ cells (bottom) among total immune cells. $\mathbf{h}$, Frequency of $\mathrm{CD} 4^{+}$and $\mathrm{CD} 8^{+} \mathrm{T}$ cells among total immune cells colored by specimen origin. Line represents the median. $\mathbf{i}$, Linear relationship between the CD4+ $T$ cell/CD8 ${ }^{+} T$ cell ratio and $11 \mathrm{~b} / \mathrm{c}^{+} 206^{+}$macrophage/CD14 ${ }^{+}$ monocyte ratio. Linear regression (black solid line) with $95 \%$ confidence interval ( $\mathrm{Cl}$; black dashed line) displayed. Significance was established with a t test (two tailed). Unless specified, all other $P$ values were calculated with a Wilcoxon rank-sum test (two tailed) $\left({ }^{\star} P<0.05 ;{ }^{\star \star} P<0.01 ;{ }^{\star \star \star} P<0.001\right)$. Coll, collagen-1; mac, macrophage; mono, monocyte; MPO, myeloperoxidase; ROI, region of interest; VIM, Vimentin. 
a
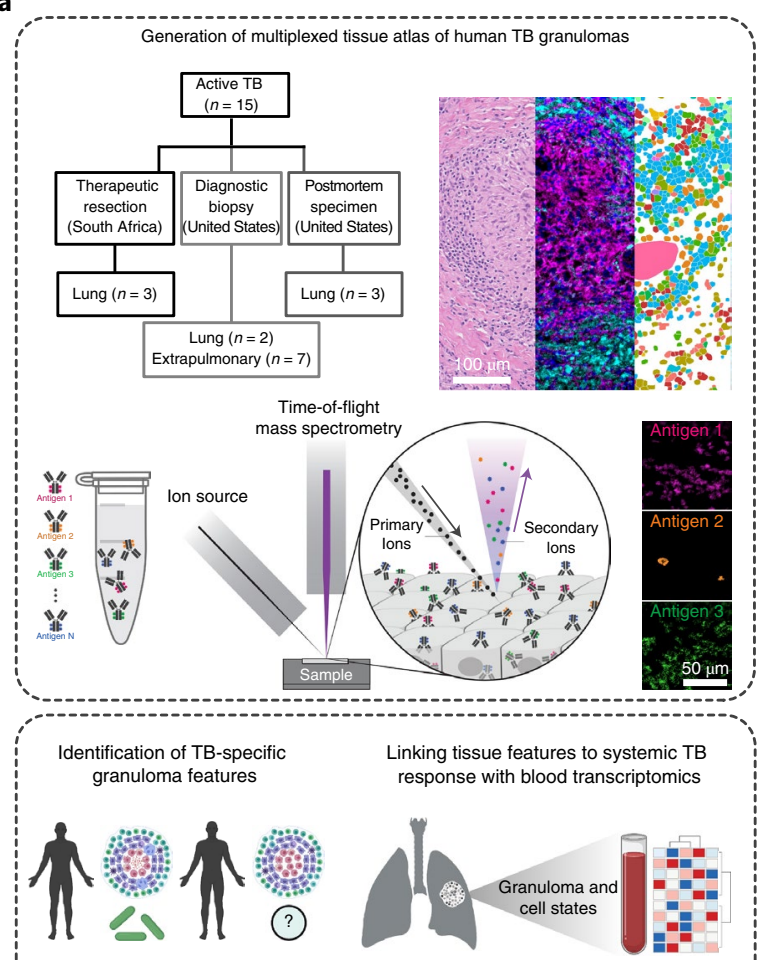

TB versus sarcoidosis

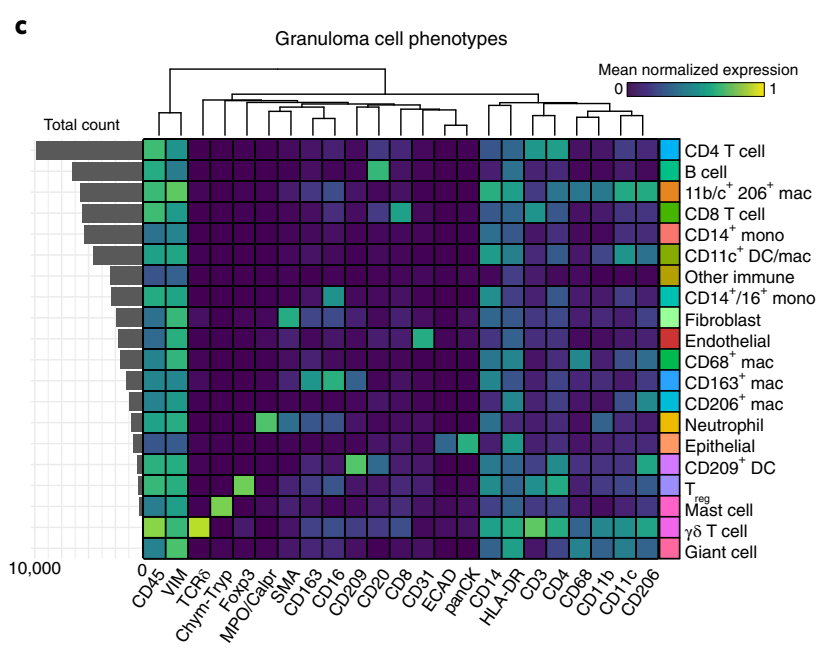

$\mathbf{f}$

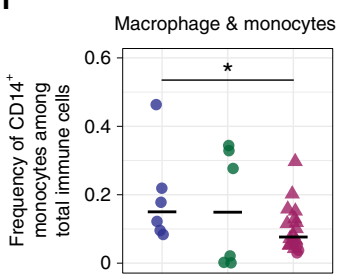

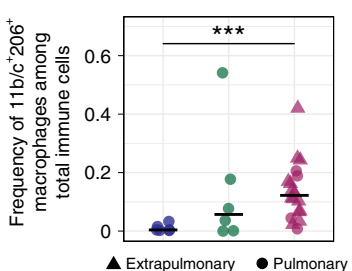

b
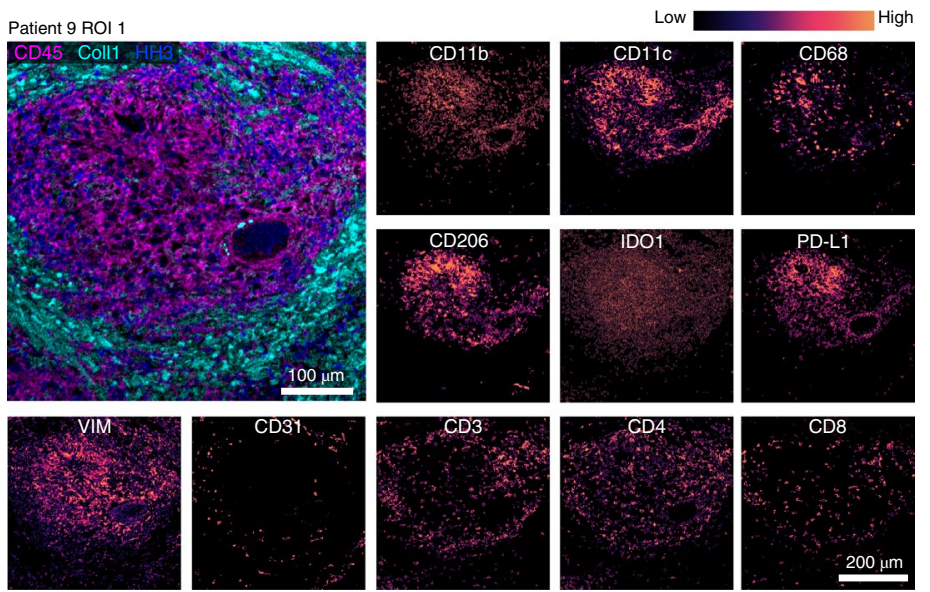

d Cell phenotype map (CPM)
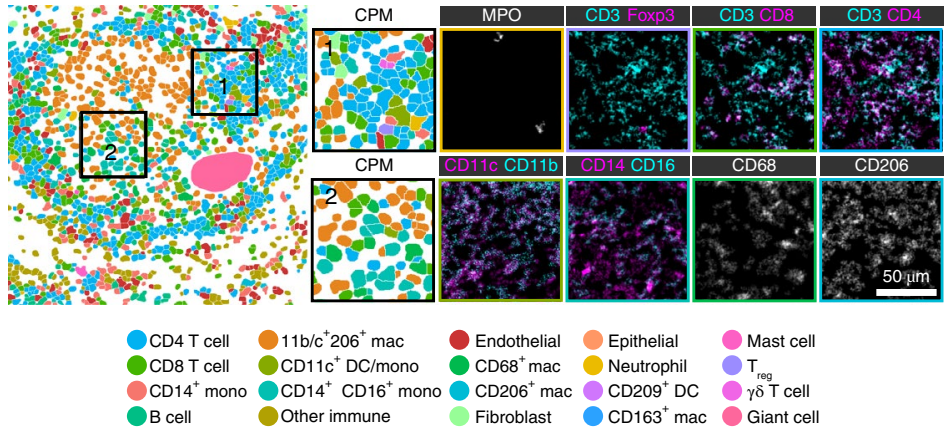

e

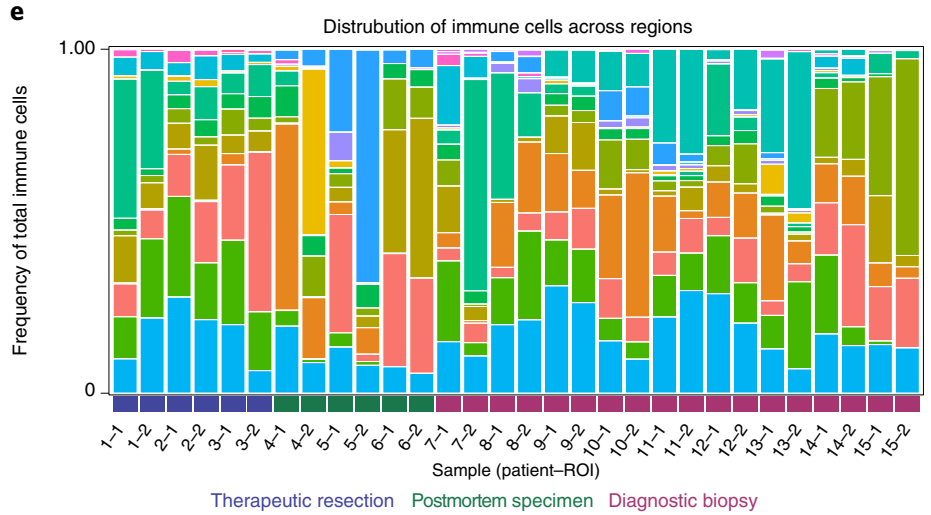

Therapeutic resection Postmortem specimen Diagnostic biopsy

h
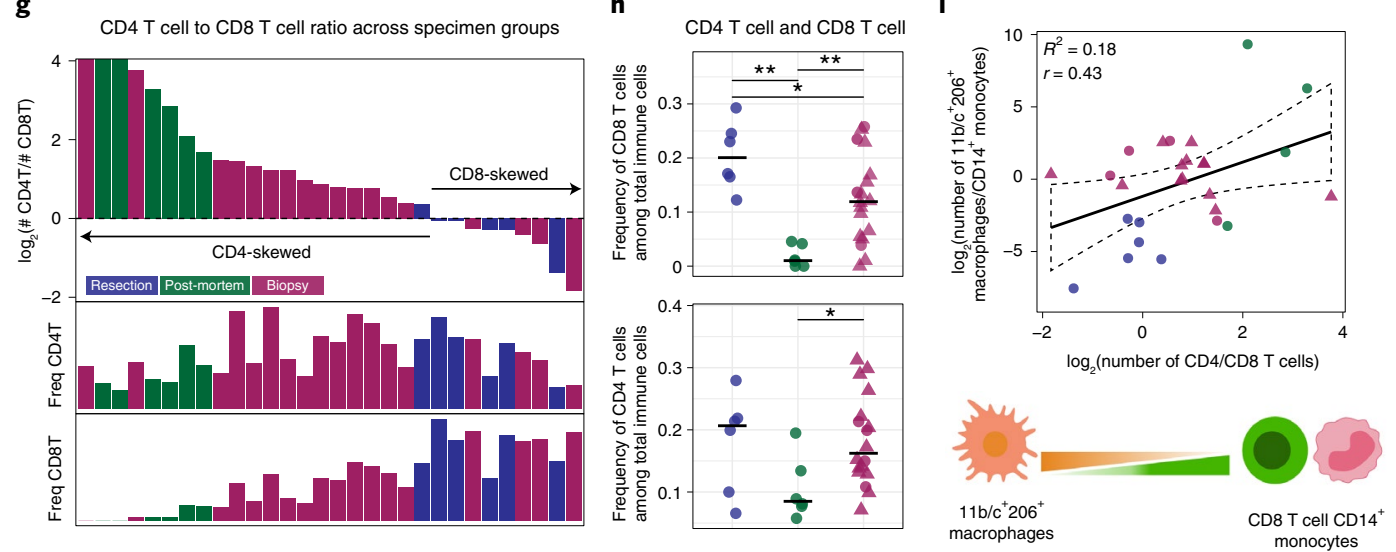
Mapping spatially coordinated biological responses in TB granulomas. To examine how granuloma structure and function are interrelated, we conducted a spatial enrichment analysis that quantified the degree of co-occurrence between protein pairs (Extended Data Fig. 3a) (ref. $\left.{ }^{18}\right)$. Enrichment scores were used to construct an interaction network that was analyzed using a community detection algorithm ${ }^{24}$ (Fig. 2a). This revealed three spatial modules consistent with canonical granuloma structures, including the myeloid core, lymphocytic cuff and stromal compartment. Intriguingly, these modules also revealed granular, previously unknown features linking cell function to spatial organization, such as association of the lymphocytic cuff with H3K9Ac and the myeloid core with IDO1 and PD-L1. Notably, this linkage was present irrespective of specimen type, suggesting granuloma structure and function are coupled and conserved within these compartments (Fig. 2a).

These findings motivated us to assess how single-cell function and granuloma structure are connected. Therefore, we employed spatial latent Dirichlet allocation (spatial-LDA) ${ }^{25}$ to discover and assign cellular MEs to each cell, where an ME is defined by cell types spatially co-occurring across the cohort (Fig. 2b). Using this approach, we identified eight MEs for summarizing the local frequency of cell subsets within a $50-\mu \mathrm{m}$ radius of a target cell (Fig. $2 \mathrm{~b}, \mathrm{c}$ ). We then labeled each cell with its highest-probability ME to generate a maximum probability map (MaxPM; Fig. 2c and Extended Data Fig. 3b). Through this approach, granuloma composition and structure were summarized with two spatial representations, a CPM and MaxPM, where cells are labeled by cell type or ME, respectively (Fig. 2c).

This allowed us to annotate canonical features of granuloma histology in an unbiased fashion while revealing previously unrecognized niches (Fig. 2d,e). The majority of granuloma macrophages and monocytes belonged to one of three myeloid MEs $\left(\mathrm{ME}_{\mathrm{Mcore}}\right.$, $\mathrm{ME}_{\text {IntMono }}$ and $\left.\mathrm{ME}_{\mathrm{Mcore} 2}\right) . \mathrm{ME}_{\mathrm{Mcore} 1}$ and $\mathrm{ME}_{\mathrm{Mcore} 2}$ were found to some degree across all specimen types, whereas $\mathrm{ME}_{\text {IntMono }}$ was notably enriched in extrapulmonary diagnostic biopsy specimens (Fig. $2 \mathrm{f}$ and Extended Data Figs. 3d,e and $2 \mathrm{~h}$ ). $\mathrm{ME}_{\mathrm{Mcore}}$ exhibited the strongest preference for the sharply demarcated granuloma core region (Extended Data Fig. 3c). In contrast, $\mathrm{ME}_{\mathrm{Mcore}}$ exhibited diffuse distribution of $\mathrm{CD} 14^{+}$monocytes and was enriched in therapeutic resections relative to pulmonary biopsy specimens, where $\mathrm{ME}_{\mathrm{Mcore}}$ was prevalent (Fig. 2d,e). In line with this, $\mathrm{ME}_{\mathrm{Mcore}}$ was highly enriched in extrapulmonary tissues and moderately to highly abundant in postmortem specimens (Extended Data Fig. 3e). Lastly, $\mathrm{ME}_{\text {IntMono }}$ exhibited the lowest preference for the canonical myeloid core and was enriched for $\mathrm{CD} 14^{+} \mathrm{CD} 16^{+}$intermediate monocytes (Fig. 2d,e and Extended Data Fig. 3c).

Next, we annotated two lymphoid MEs, the lymphocytic cuff $\left(\mathrm{ME}_{\mathrm{Lcuff}}\right)$ and tertiary lymphoid structures $\left(\mathrm{ME}_{\mathrm{TLS}}\right) . \mathrm{ME}_{\mathrm{Lcuff}}$ aligned with the second canonical granuloma $\mathrm{ME}$ (the lymphocytic cuff) and was composed of $\mathrm{CD}^{+}$and $\mathrm{CD}^{+} \mathrm{T}$ cells (Fig. $2 \mathrm{~d}, \mathrm{e}$ ). $\mathrm{ME}_{\mathrm{TLS}}$ was predominated by $\mathrm{B}$ cells, with sparse numbers of follicular helper T cells $\left(\mathrm{CD}^{+} \mathrm{PD}-1^{+}\right)$, consistent with TLSs (confirmed by hematoxylin and eosin (H\&E); Fig. 2e) (refs. ${ }^{26,27}$ ). This ME was highly abundant in FOVs that were B cell enriched across specimen groups (Figs. 1e and 2f).

In our composition analysis, we observed that some granulomas exhibited a fibrotic wound-healing response with fibroblasts and
$\mathrm{CD}_{163}+\mathrm{M} 2$-like macrophages (Fig. 1e). Spatial-LDA revealed these cells colocalized within ME fibrosis $\left(\mathrm{ME}_{\mathrm{Fib}}\right)$, where $\mathrm{CD} 36$, a fibroblast marker, and collagen-1, a marker for fibrosis, were expressed (Fig. 2d,e $)^{28}$. The last two MEs represented less characterized cellular environments in $\mathrm{TB}$ infection. $\mathrm{ME}$ vasculature $\left(\mathrm{ME}_{\text {Vasc }}\right)$ was predominated by blood vessels, neutrophils and mast cells, whereas $\mathrm{ME}_{\mathrm{Epi}}$ (lung parenchyma) was composed of IFN- $\gamma^{+}$ epithelial cells and CD206 $6^{+}$alveolar-like macrophages (Fig. 2d,e). Given that these cells are known to participate in angiogenesis, tissue repair, and immune cell recruitment ${ }^{29}$, perivascular localization of mast cells in the granuloma could suggest their involvement in these processes, especially considering the association between mast cell quantity and local bacterial burden ${ }^{30}$. On the other hand, because $\mathrm{ME}_{\text {Vasc }}$ was modestly lower in extrapulmonary biopsy specimens than pulmonary biopsy specimens $(P=0.06$, Wilcoxon rank sum) and significantly lower than pulmonary resections $(P=0.04)$ (Fig. $2 f$ and Extended Data Fig. 3d,e), this result may reflect organ-specific differences in the association between vascularity and granulocytes or abundance of tissue resident mast cells.

Given the association between granuloma composition and clinical origin, we next sought to determine whether this relationship applied to granuloma structure. Using a correlation-based approach, we found that five ME frequency clusters accounted for $81 \%$ of variance in our dataset (Fig. $2 \mathrm{f}$ and Extended Data Fig. 3f). Notably, four out of five of these clusters contained samples from more than one group, supporting a recurrent spatial framework where granuloma composition and structure are coupled in a manner that is clinically agnostic (Fig. 2f). Altogether, this result suggests that MEs capture spatial features that are not discernible by bulk cell composition alone and indicate spatially coordinated biological responses.

\section{Granuloma myeloid cells express an immunoregulatory program.} Spatial modeling of granulomas revealed myeloid-rich regions of the granuloma are characterized by expression of IDO1 and PD-L1 (Fig. 2a,d). Given the tolerogenic role of these proteins ${ }^{31-35}$, we sought to characterize the cellular and spatial nature of immunoregulatory phenotypes in the myeloid compartment. Coexpression of PD-L1 and IDO1 was correlated (Pearson $r=0.67, P<2.2 \times 10^{-16}$, $t$ test) across 11 granulocyte, macrophage, monocyte and DC populations (Fig. 3a,b and Extended Data Fig. 4a,b) and was highest in CD $11 b^{+} \mathrm{CD} 11 \mathrm{c}^{+}$macrophages (Fig. $3 \mathrm{~b}$ and Extended Data Fig. $4 \mathrm{~b}$ ), a phenotype identical to that of a recently described immunosuppressive tumor-associated macrophage ${ }^{36}$. $\mathrm{CD}_{16}{ }^{+} \mathrm{CD} 14^{+}$intermediate monocytes exhibited a bimodal distribution in which PD-L1 and IDO1 associated with HLA-DR downregulation, which is consistent with immune evasion that disables antigen presentation to $\mathrm{CD}^{+} \mathrm{T}$ cells (Fig. $3 \mathrm{~b}$ and Extended Data Fig. 4b) (ref. ${ }^{37}$ ). With respect to clinical origin, $\mathrm{PD}-\mathrm{L} 1$ and IDO1 expression was correlated in all specimens but only weakly correlated in resections because of PD-L1 depletion (Extended Data Fig. 4c,d). Notably, neutrophils also expressed IDO1 or PD-L1 (Extended Data Fig. 4e). Taken with previous work identifying neutrophils that secrete anti-inflammatory cytokines in TB granulomas ${ }^{38}$, these findings align with a regulatory effector function. Lastly, nearly 100\% of MNGCs expressed IDO1, and $\sim 85 \%$ expressed PD-L1, a feature present across specimen groups and organ site (Fig. 3e and Extended Data Fig. 4f).

Fig. 2 | Spatial analysis of granuloma protein expression and cellular MEs. a, Positive spatial enrichments (average z-score $>0$ ) between protein pairs as a weighted, undirected network (edge weight is proportional to average $z$-score) with three communities (myeloid core, green; lymphocytic cuff, blue; nonimmune/other, pink). b, Conceptual overview of spatial-LDA. c, Cell probability map (left), max probability map (right), and ME probability for 8 MEs (middle, scaled 0 to 1) for a representative TB granuloma. d, Heatmap of ME preferences for all subsets (standardized mean ME loading) with hierarchical clustering (Euclidean distance, complete linkage) and mean normalized expression of functional markers (probability weighted mean) with columns hierarchically clustered (Euclidean distance, complete linkage). e, Biological classification of MEs. f, Frequency of all MEs per FOV. Heatmap columns are hierarchically clustered (Pearson correlation, complete linkage). Paired ROls from the same patient annotated with a black bar. ME cluster and sample clinical origin annotated below dendrogram. ExPulm, extrapulmonary; MC, mast cell; pulm, pulmonary; HH3, histone H3; Pan-CK, pan-cytokeratin. 
To assess how PD-L1 and IDO1 expression varies with location in the granuloma, we calculated the frequency of PD-L1+ and $\mathrm{IDO}^{+}$nongranulocytic myeloid cells in each ME (Fig. $3 \mathrm{f}-\mathrm{h}$ and
Extended Data Fig. 4g). We found the majority of cells displayed preferential, ME-specific expression that was independent of subset frequency. For example, the frequency of PD-L1-expressing

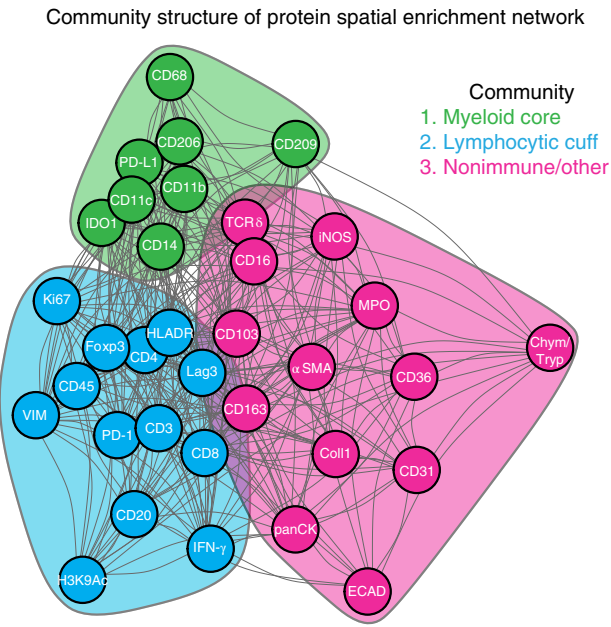

b

Spatial-LDA overview
C

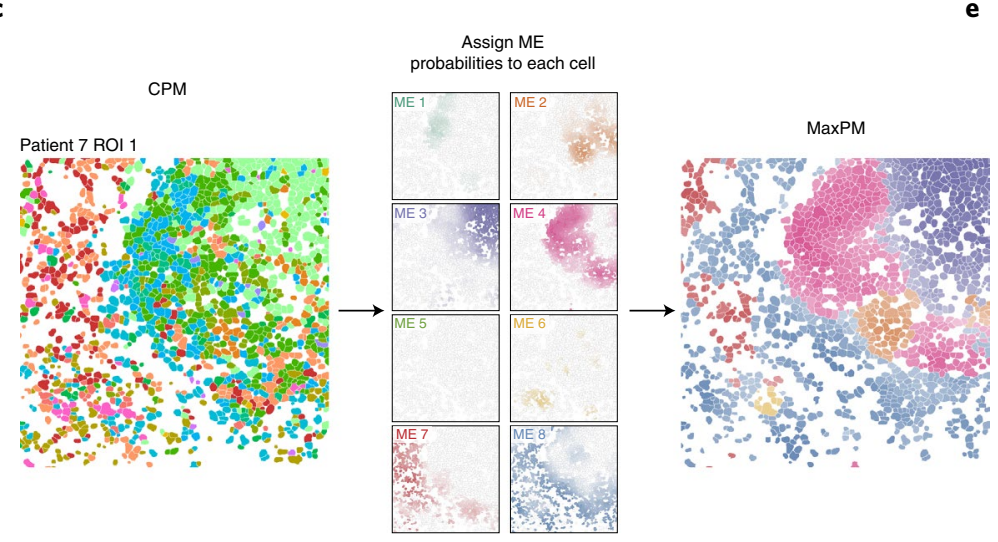

d Cell type preferences and marker expression across MEs
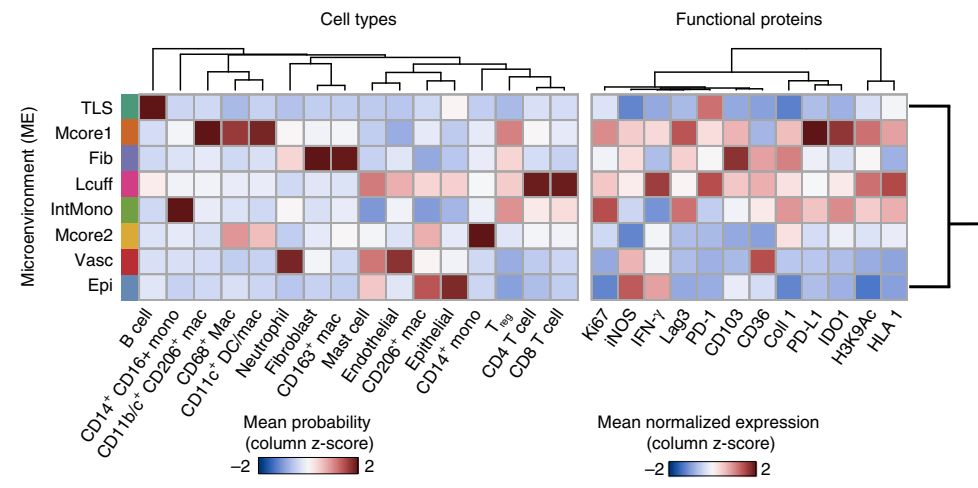

f

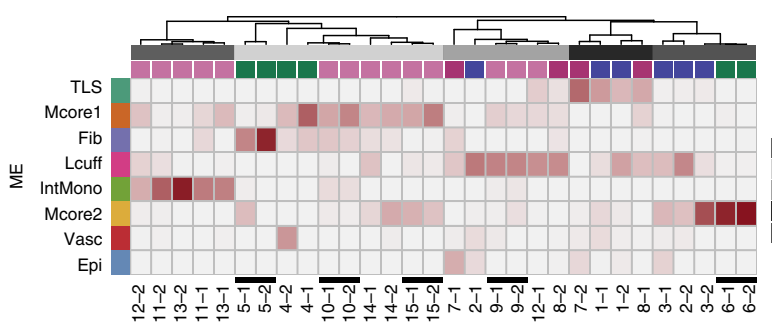

Sample (patient-ROI)
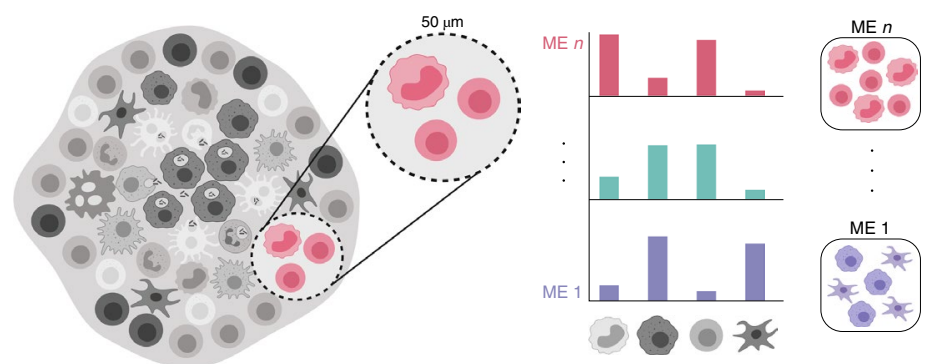

$\mathrm{ME}=$ cell subsets cooccuring in a spatially defined proximity $M E_{\text {Mcore1 }} \mathrm{ME}_{\text {IntMono }} \mathrm{ME}_{\text {Mcore2 }}$ Patient 12 ROI 2

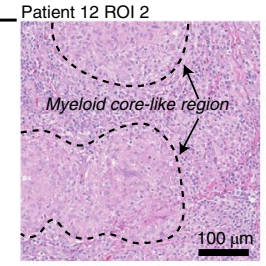

$\mathrm{ME}_{\text {Lcuff }}$

Patient 9 ROI 1

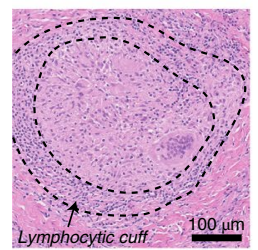

Lymp

Patient 7 ROI 2
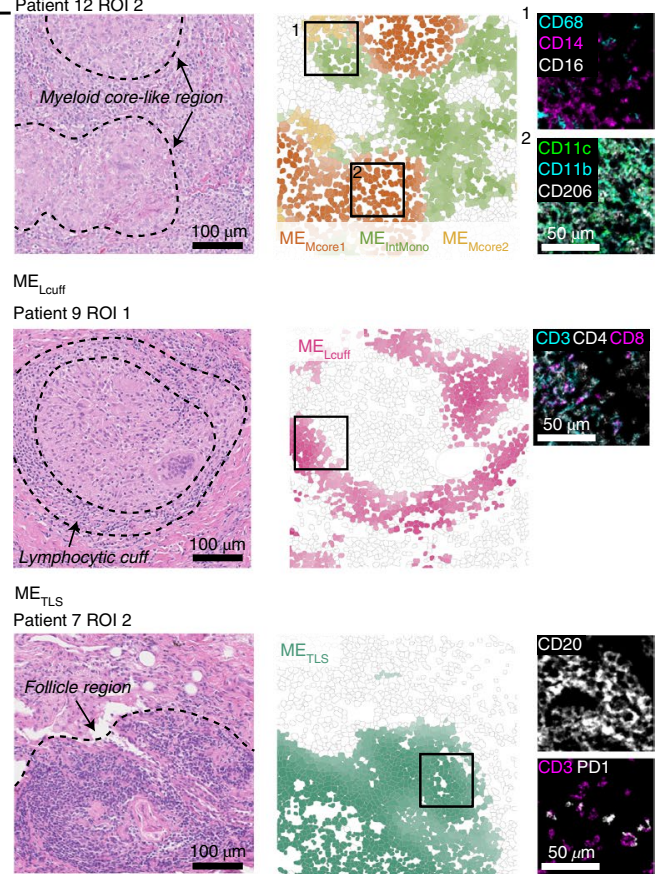

$\mathrm{ME}_{\mathrm{Fib}}$

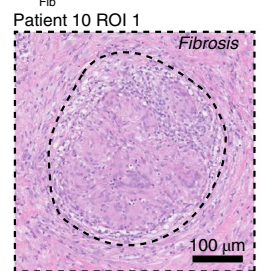

$\mathrm{ME}_{\text {Vasc }}$

Patient 1 ROI 1

ME 1 \%

$M E_{\text {Vase }}$

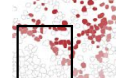

एकी

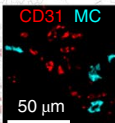

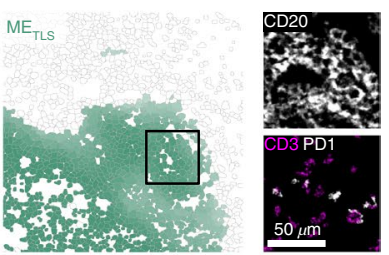

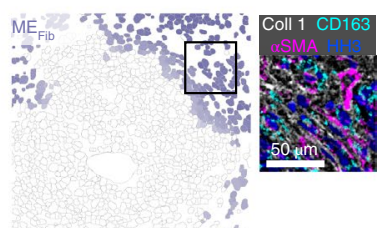

$\mathrm{ME}_{\mathrm{Epi}}$

Patient 2 ROI 1

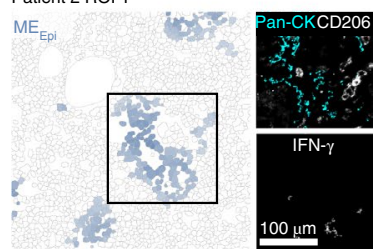


a

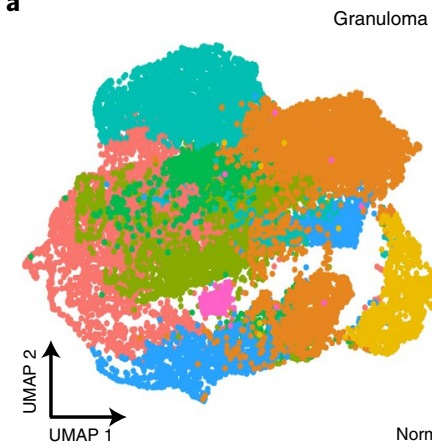

ranuloma myeloid cell expression profiles

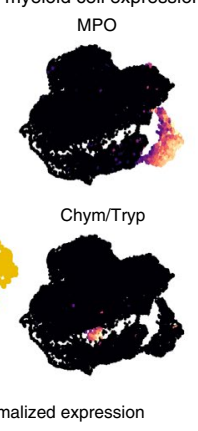

$0 \square 1$

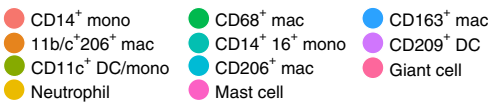

d

IDO1 and PD-L1 correlation

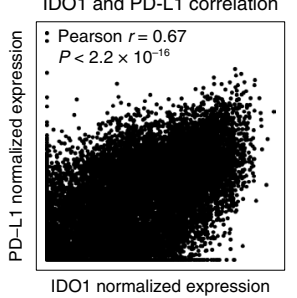

Expression of IDO1 and PD-L1 by giant cells
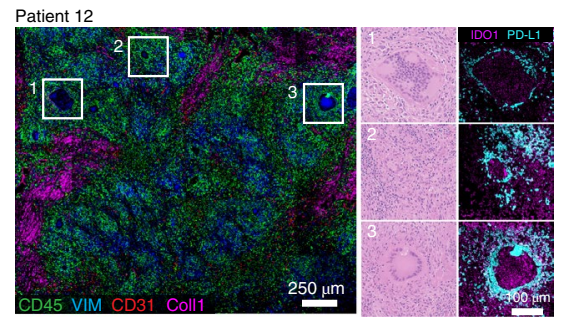

b

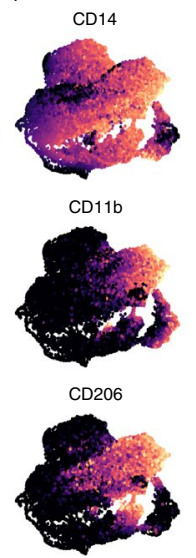

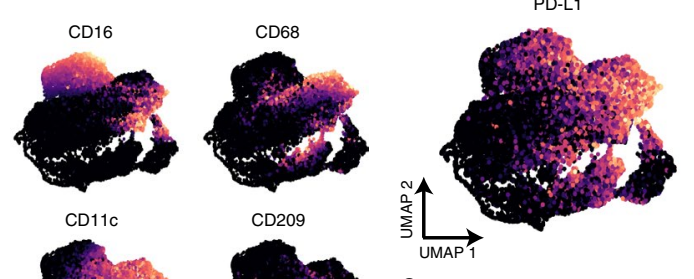

Patient 9 ROI 2

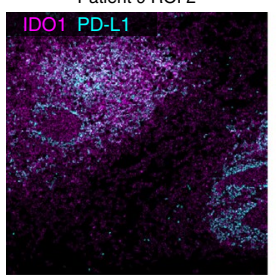

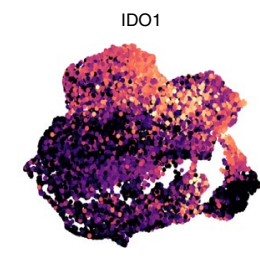

Patient 10 ROI 2

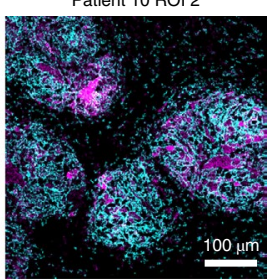

f

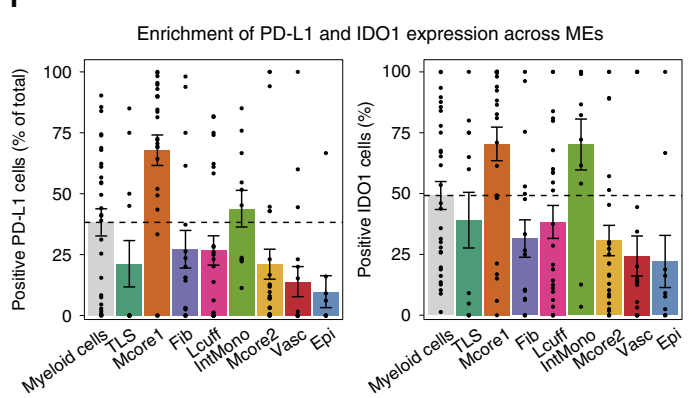

g

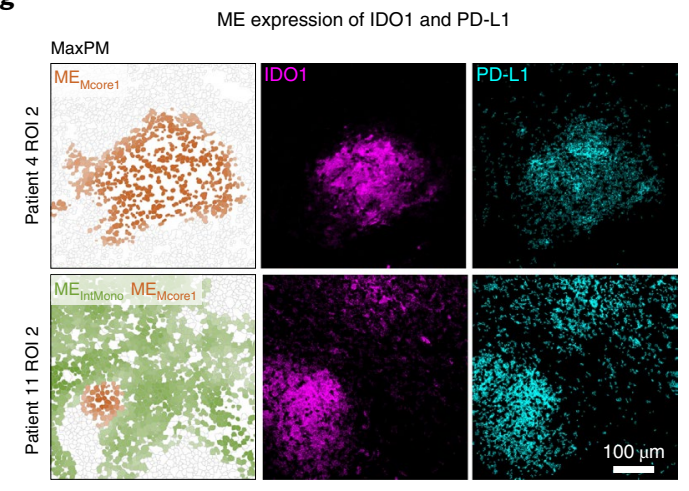

h

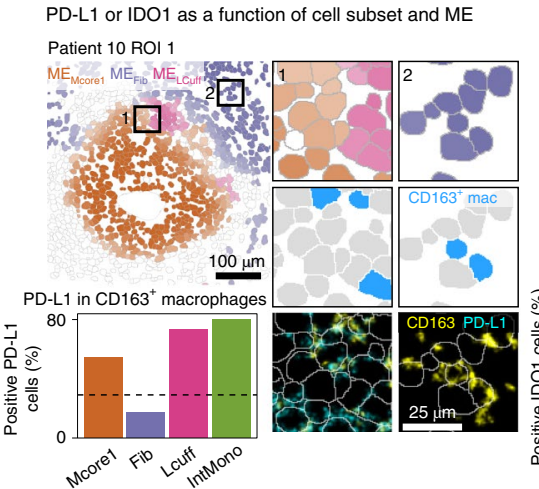

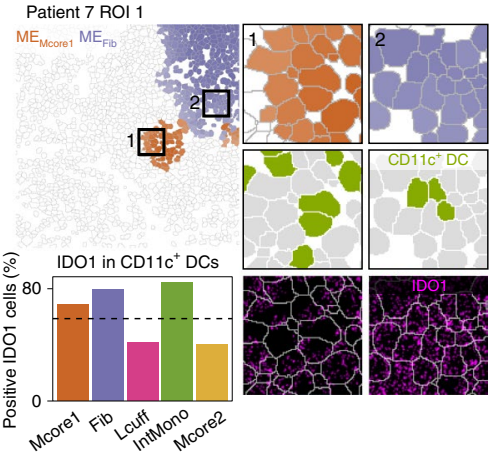

Fig. 3 | Granuloma myeloid cells express a spatially coordinated immunoregulatory program. a, UMAP visualization of all myeloid populations across all TB FOVs colored by subset (left) and normalized expression of phenotypic markers used to delineate subsets. b, IDO1 and PD-L1 normalized expression overlaid on the UMAP. c, Representative images of TB granulomas showing expression of IDO1 (magenta) and PD-L1 (cyan). d, PD-L1 and IDO1 expression values across all myeloid cells as a biaxial scatter plot. Plot displays Pearson's $r$ and $P$ value calculated by a $t$ test (two tailed). e, Giant cells identified from a MIBI-scanned TB sample (CD45, green; Vimentin, blue; CD31, red). Representative giant cells displayed in zoomed insets (lower left) with H\&E staining or IDO1 (magenta) and PD-L1 (cyan) expression. Bar plot displays the percentage of IDO1+ and PD-L1+ giant cells $(n=34$, normalized expression $>0$ ).

f, The frequency of $\mathrm{IDO}^{+}$and PD- $\mathrm{L}^{+}$nongranulocytic myeloid cells in aggregate and broken down by ME. Bars represent mean \pm s.e.m. ( $n=30$ ). g, $\mathrm{ME}_{\mathrm{Mcore1}}$ and $\mathrm{ME}_{\text {IntMono }}$ maximum probability maps and representative images of a pulmonary (top) and pleural (bottom) TB sample showing expression of IDO1 (magenta) and PD-L1 (cyan). h, Frequency of PD-L1+ CD163+ macrophages (left) across MEs with a representative MaxPM. Insets are colored by ME (top), cell type (blue, middle) and CD163 (yellow) and PD-L1 (cyan), with the segmentation boundaries overlaid (white). The frequency of IDO1+ CD11 ${ }^{+}$DCs (right) across MEs with a representative MaxPM. Insets are colored by ME (top), cell type (green, middle) and IDO1 (magenta), with the segmentation boundaries overlaid (white). Dashed lines represent the total frequency of positive cells (PD-L1 or IDO1) for the indicated cell subset. HLA, human leukocyte antigen; HLA-DR-DQ-DP, HLA-DR/HLA-DQ/HLA-DP.

$\mathrm{CD}_{163}{ }^{+}$macrophages was highest in $\mathrm{ME}_{\mathrm{Mcorel}}$ (53.5\%), $\mathrm{ME}_{\mathrm{Lcuff}}$ (71.6\%) and $\mathrm{ME}_{\text {IntMono }}(78.3 \%)$, despite this population being most prevalent in $\mathrm{ME}_{\mathrm{Fib}}$ (Fig. 3h). Similarly, IDO1-expressing CD11c $\mathrm{c}^{+}$ DCs were most enriched in $\mathrm{ME}_{\mathrm{Fib}}(78.9 \%)$ and $\mathrm{ME}_{\text {IntMono }}(83.7 \%)$ (Fig. 3h). Altogether, PD-L1 and IDO1 coexpression defines a newly identified, spatially coordinated immunoregulatory feature of TB granulomas. Given the observational nature of this study, a functional role cannot be directly evaluated. However, these data support the possibility of highly localized, myeloid-mediated immune suppression in the granuloma. 

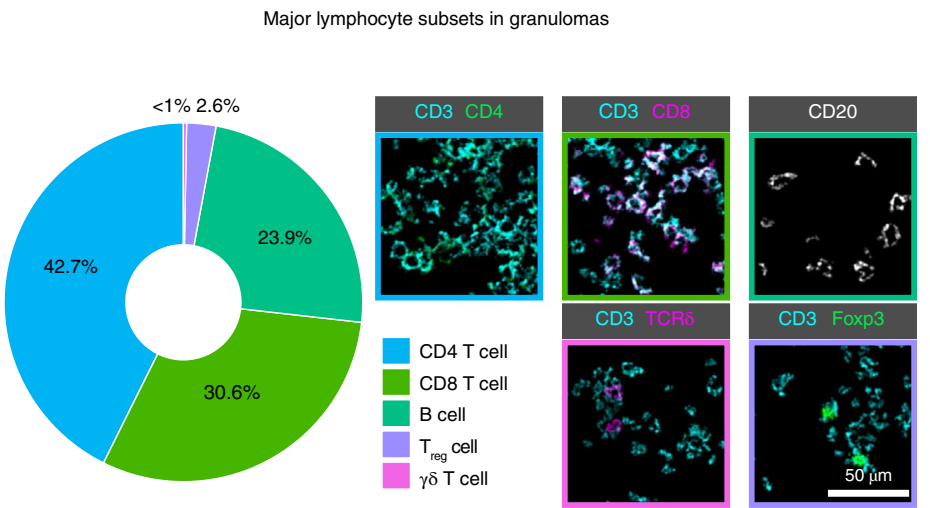

b

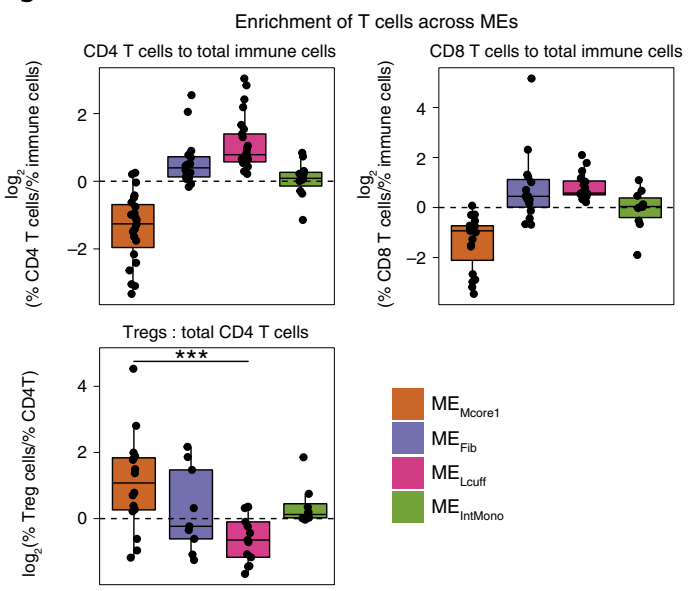

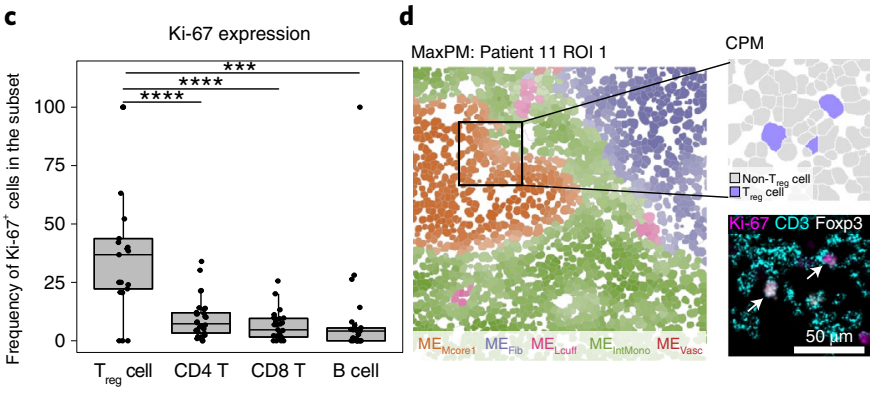

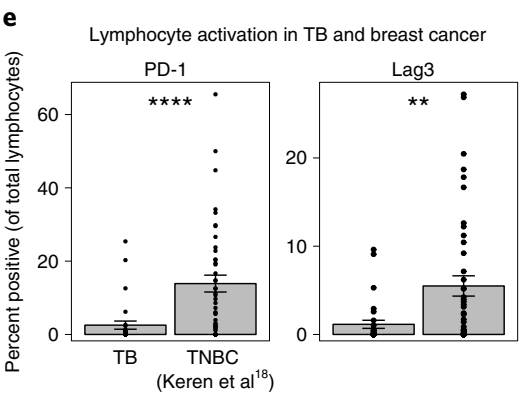

$f$

Fig. 4 I Granuloma lymphocytes display a paradoxical absence of exhaustion markers. a, Frequency of lymphocyte subsets in all TB FOVs pooled together (left) and representative images of each subset (right). $\mathbf{b}$, Frequency of $\mathrm{CD}^{+}{ }^{+}$and $\mathrm{CD} 8^{+} \mathrm{T}$ cells relative to the frequency of total immune cells in four MEs of interest (top). The frequency of $\mathrm{T}_{\text {reg }}$ cells relative to the frequency of total $\mathrm{CD} 4^{+} \mathrm{T}$ cells (lower left) $(n=30)$. $\mathbf{c}$, Frequency of Ki-67+ cells broken down by lymphocyte subset $(n=30)$. d, Representative image of a TB granuloma, colored by ME assignment (left). Zoomed inset displays $T_{\text {reg }}$ cell assignment (upper right: purple, $T_{\text {reg }}$ cell; gray, non- $T_{\text {reg }}$ cell) and expression of Ki-67 (magenta), CD3 (cyan) and Foxp3 (white) (lower right). e, Percentage of lymphocytes positive for PD-1 (left) and Lag3 (right) in all TB FOVs and TNBC. Bars represent mean \pm s.e.m. (TB $n=30$, TNBC $n=43$ ). f, The ratio of PD-1+ to PD-L1+ immune cells represented as a $\log _{2}$ fold change in all TB FOVs and TNBC (TB $\left.n=30, T N B C n=43\right)$. Boxplots display the median and interquartile range (IQR; $25-75 \%)$ with whiskers representing the upper- and lower-quartile $\pm 1.5 \times I Q R$. All $P$ values were calculated with a Wilcoxon rank-sum test (two tailed) $\left({ }^{\star \star} P<0.01 ;{ }^{\star \star \star} P<0.001 ;{ }^{\star \star \star \star} P<0.0001\right)$.

Granuloma lymphocytes are sparsely activated. We next wanted to evaluate to what extent the coordination between structure and function observed in myeloid cells extended to lymphocytes (Fig. 4a). On comparing the proportion of $\mathrm{T}$ cell subsets within each $\mathrm{ME}, \mathrm{T}_{\text {reg }}$ cells $\left(\mathrm{CD}^{+} \mathrm{CD} 4^{+} \mathrm{Foxp}^{+}\right)$were uniquely enriched in $\mathrm{ME}_{\mathrm{Mcore}}$ relative to the lymphocytic cuff (Fig. $4 \mathrm{~b}(P=0.0007$, Wilcoxon rank sum)), and the total number of $\mathrm{ME}_{\mathrm{Mcore}}$-infiltrating $\mathrm{T}_{\text {reg }}$ cells was correlated to the number of $\mathrm{IDO}^{+}$cells $\left(R^{2}=0.32\right.$, $P=0.003, t$ test $)$ and PD-L1 ${ }^{+}$cells $\left(R^{2}=0.33, P=0.003\right)$ in this environment (Extended Data Fig. 4h). All other lymphocyte subsets were enriched in $\mathrm{ME}_{\text {Lcuff }}$ including Foxp3 ${ }^{-} \mathrm{CD} 4^{+} \mathrm{T}$ cells (Fig. 4b). Furthermore, the frequency of proliferating $\mathrm{T}_{\text {reg }}$ cells exceeded that of all other lymphocyte subsets (Fig. 4c, $P<0.001$, Wilcoxon rank sum). Taken together, these findings suggest that $\mathrm{T}_{\text {reg }}$ cells, including those actively proliferating, and immunoregulatory myeloid cells colocalize in $\mathrm{ME}_{\mathrm{Mcore}}$ to potentiate an immunomodulatory niche (Fig. 4d) (refs. ${ }^{39-42}$ ).

Anti-inflammatory pathways can be induced as negative feedback that moderates the cytotoxic effects of unchecked immune activation $^{43}$. In line with this, high expression of PD-L1 and IDO1 by granuloma myeloid cells would be expected to be accompanied by $\mathrm{T}$ cell activation in the form of checkpoint expression (e.g., PD-1 and Lag3) (ref. ${ }^{44}$ ). For example, when examining infiltrated triple-negative breast cancer (TNBC) tumors, we found the median ratio of PD- $1^{+}$to $\mathrm{PD}-\mathrm{L1}^{+}$immune cells to be near unity (Fig. 4f) and the prevalence of $\mathrm{PD}-1$ or Lag3 positive lymphocytes to be $13.9 \%$ and $5.5 \%$ on average, respectively (Fig. $4 \mathrm{e}$ ).

Surprisingly, a relationship consistent with compensatory negative feedback was not observed here. We found PD-L1+ granuloma immune cells far outnumbered PD- $1^{+}$immune cells $\left(\log _{2}\left[\mathrm{PD}-1^{+} /\right.\right.$ $\left.\mathrm{PD}-\mathrm{L}^{+}\right]=-5.1 \pm 3.5$; Fig. $\left.4 \mathrm{f}\right)$. Furthermore, the small numbers of $\mathrm{PD}-1^{+}$lymphocytes were largely restricted to $\mathrm{ME}_{\mathrm{TLS}}$, consistent with $\mathrm{T}$ follicular helper cells rather than an activated phenotype (Extended Data Fig. 4i). These findings are consistent with reports from the cynomolgus macaque TB model that found low levels of PD-1, Lag3 and CTLA-4 (ref. ${ }^{45}$ ), suggesting that IDO1 and PD-L1 expression by myeloid cells could occur independently of local signaling by activated $\mathrm{T}$ cells.

Considering recent work demonstrating TGF- $\beta$ signaling in granulomas ${ }^{46}$, the combination of tolerogenic myeloid cells and $\mathrm{T}_{\text {reg }}$ cells along with the absence of $\mathrm{T}$ cell activation could indicate an immunoregulatory niche promoted through TGF- $\beta$. To measure the cytokine landscape underlying this ME, we performed in situ hybridization (ISH) for TGF- $\beta$ and IFN- $\gamma$ transcripts in a subset $(n=3)$ of samples (Extended Data Figs. 5 and 6). Consistent with measurements by MIBI-TOF, granulomas were depleted of IFN- $\gamma$ and produced large quantities of TGF- $\beta$ (Extended Data Figs. $4 \mathrm{j}$ and $5 \mathrm{a}-\mathrm{g}$ ). The majority of TGF- $\beta$ was produced by cells in the myeloid core, corresponding with expression of IDO1 and PD-L1 (Extended Data Fig. 5d). However, TGF- $\beta$ was also produced by cells in the 


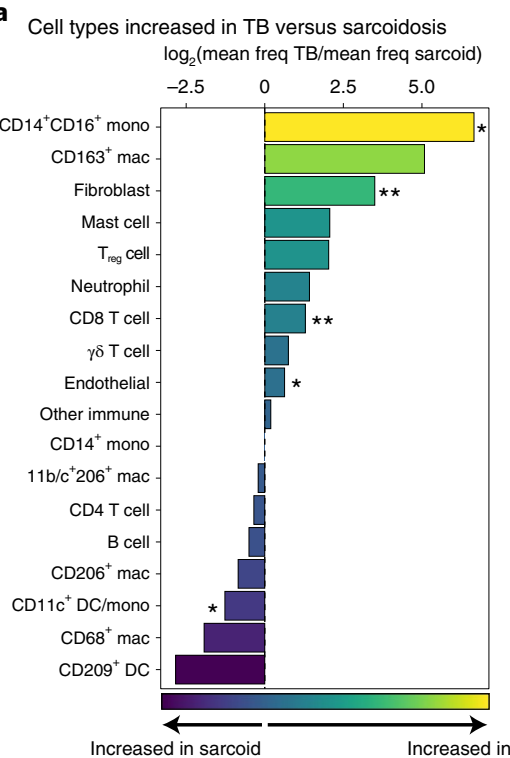

b
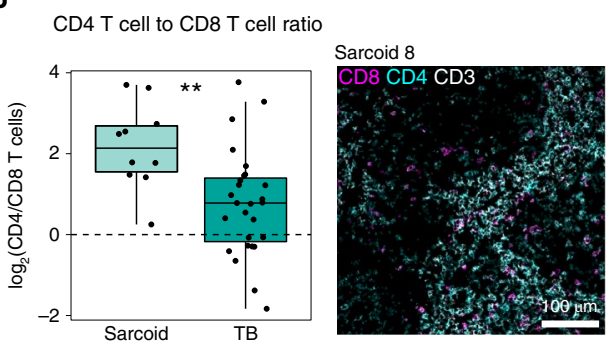

d

Fig. 5 | Common and diverging features of immune regulation in TB and sarcoidosis. a, Fold change of mean frequency of cell subsets (of total cells) in $T B$ versus sarcoidosis with significant differences indicated with an asterisk. $\mathbf{b}$, Comparison of the $C D 4^{+} T$ to $C D 8^{+} T$ cell ratio in TB versus sarcoidosis (TB $n=30$, sarcoid $n=10$ ). Representative image of an axillary sarcoidosis FOV showing expression of CD8 (magenta), CD4 (cyan) and CD3 (white) (left) and colored by cell type (right: blue, CD4+ T cell; green, CD8 ${ }^{+} \mathrm{T}$ cell). c, Percentage of lymphocytes positive for PD-1 (top) and Lag3 (bottom) in all sarcoidosis FOVs, TB FOVs and TNBC. Bars represent mean \pm s.e.m. (sarcoid $n=10, T B n=30$, TNBC $n=43$ ). d, Percentage of total cells positive for IDO1 or PD-L1 in TB and sarcoidosis (TB $n=30$, sarcoid $n=10$ ). Representative image of a sarcoidosis FOV showing expression of PD-L1 (cyan) and HH3 (white). Boxplots display the median and IQR (25-75\%), with whiskers representing the upper- and lower-quartile $\pm 1.5 \times I Q R$. All $P$ values were calculated with a Wilcoxon rank-sum test (two tailed) (NS, $P>0.05 ;{ }^{\star} P<0.05$; ${ }^{\star \star} P<0.01$; ${ }^{\star \star \star} P<0.001 ;{ }^{\star \star \star \star} P<0.0001$ ).

lymphocytic cuff of granulomas (Extended Data Fig. 5d). There was no correlation between TGF- $\beta$ and IFN- $\gamma$ transcripts, suggesting that TGF- $\beta$ expression may occur in the absence of T helper type 1 signaling (Extended Data Fig. 5h). These results demonstrate that TGF- $\beta$ expression underlies a granuloma ME producing IDO1 and PD-L1 and promoting $\mathrm{T}_{\text {reg }}$ cell activity. Such an environment may potentiate a niche within the granuloma that impairs $\mathrm{T}$ cell activation and promotes $\mathrm{T}_{\text {reg }}$ cell proliferation.

Common and diverging immunoregulatory features in TB and sarcoidosis. In addition to being the histological hallmark of TB, granulomas occur in response to foreign bodies and in autoimmune disorders, such as sarcoidosis ${ }^{47}$. Interestingly, gene expression studies that attempted to develop blood-based biomarkers for $M t b$ infection have struggled to differentiate TB from sarcoidosis ${ }^{48,49}$. To determine the extent to which features identified here overlap with other granulomatous diseases, we compared TB to ten sarcoidosis cases (Extended Data Fig. 7a). TB lesions were more variable in composition (Extended Data Fig. 7b,c) and had significantly higher frequencies of $\mathrm{CD}^{+} \mathrm{T}$ cells, fibroblasts, intermediate monocytes and giant cells and increased vascularity (Fig. 5a and Extended Data Fig. 7c). Sarcoid granulomas were heavily $\mathrm{CD} 4^{+} \mathrm{T}$ cell skewed, even relative to CD4-skewed TB granulomas, consistent with reports of sarcoidosis pathology being driven primarily by $\mathrm{T}$ helper type 17 and type 1 T cells (Fig. 5b) (refs. ${ }^{50,51}$ ).

Like TB, sarcoid lesions were PD-1 and Lag3 depleted (Fig. 5c), despite high levels of PD-L1 ${ }^{+}$myeloid cells (Fig. 5d and Extended Data Fig. 7d). However, unlike TB, IDO1 expression in sarcoid samples was almost entirely absent (Fig. 5d). Because we used a conservative threshold for IDO1 and PD-L1 positivity, our analysis biased toward the moderately to strongly expressing cells present in TB granulomas and control tissues. Therefore, to evaluate the specificity of macrophage PD-L1 and IDO1 expression in $M t b$ infection, we used immunohistochemistry (IHC) to compare both proteins on a tissue microarray of granulomas from sarcoidosis $(n=9)$, foreign body uptake $(n=4)$, endometriosis $(n=4)$ and xanthomatosis $(n=3)$ (Extended Data Fig. 7e). We identified weak expression of IDO1 in several sarcoidosis lesions along with bright expression of PD-L1, as observed by MIBI-TOF (Extended Data Fig. 7e). However, $\mathrm{IDO}^{+}$and $\mathrm{PD}-\mathrm{L}^{+}$cells were nearly absent in all xanthomas and endometrial lesions and rare in foreign body granulomas. Notably, we observed high levels of IDO1 and PD-L1 in a pulmonary Mycobacterium avium granuloma (Extended Data Fig. 7f). This suggests that whereas PD-L1 expression could be a broader feature of granulomatous conditions, strong coexpression of IDO1 and PD-L1 appears specific to mycobacterial granulomas.

Immunoregulatory features are reflected across granulomas and blood. The presence of immunoregulatory features observed in our MIBI-TOF study has important implications for understanding the immunologic basis of TB disease. We next wanted to analyze these features in blood from TB patients to understand how immunoregulatory properties of granulomas are reflected during systemic immunity. Moreover, by leveraging analysis of blood, we sought to look across infection stages and correlate immune features with disease severity and progression. Therefore, we used MetaIntegrator to perform multicohort analyses using publicly available peripheral blood transcriptome profiles from healthy subjects and patients with latent or active TB infection ${ }^{52,53}$.

We first asked whether immunoregulatory signals identified in granulomas could be detected in blood by comparing gene expression data of patients with active TB $(n=647)$ to healthy controls $(n=197)$ from 13 independent cohorts (Fig. 6a). We found significant and consistent upregulation of IDO1 and CD274 (PD-L1) (effect size $=0.77$ and $1.28, q=0.0009$ and 0.006 (false discovery rate $=5 \%$ ), respectively) (Fig. 6b). Additionally, checkpoint depletion in lymphoid cells was corroborated, with no observed increase 
Whole-blood gene expression meta-analysis

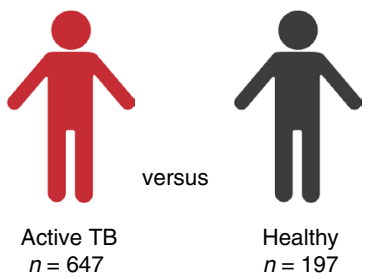

(13 cohorts)
IDO1

Effect size $=0.77$ Adjusted $P=0.0009$

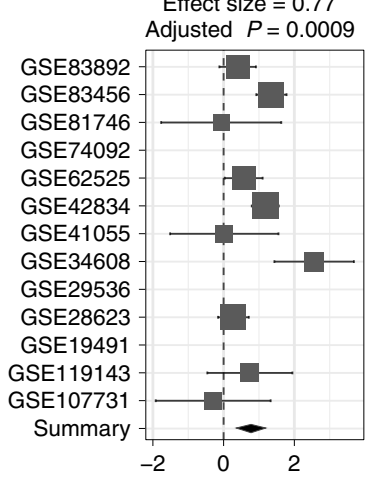

Active TB versus healthy CD274 (PD-L1)

Effect size $=1.28$ Adjusted $P=0.006$

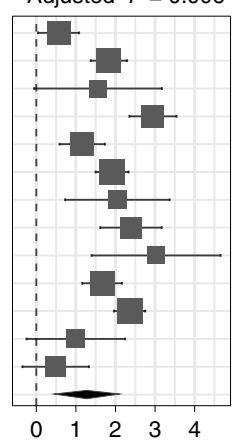
PDCD1 (PD-1)

Effect size $=-0.41$ Adjusted $P=0.08$

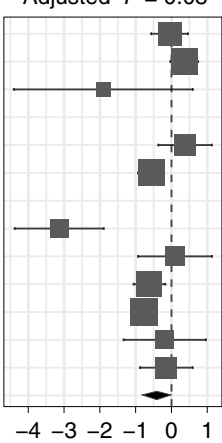

Standardized mean difference $\left(\log _{2}\right.$ scale)
LAG3

Effect size $=-0.39$ Adjusted $P=0.05$

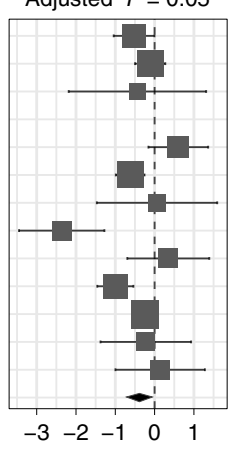
Meta-analysis across infection stages
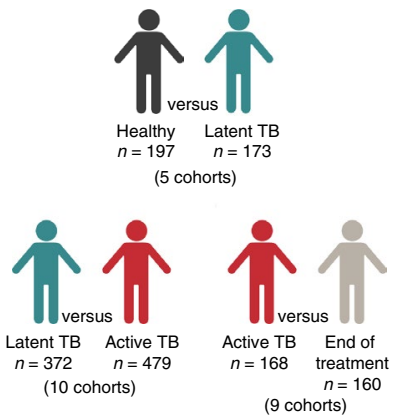

Adolescent cohort study (ACS) gene expression over disease progression

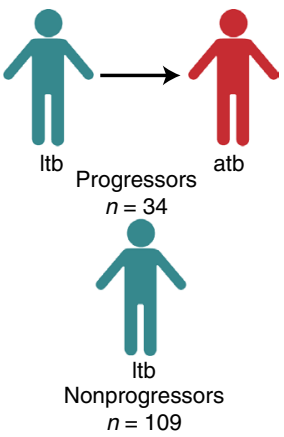

Summary effect sizes across infection stage

Mean effect size $-1.5 \square 1.5$

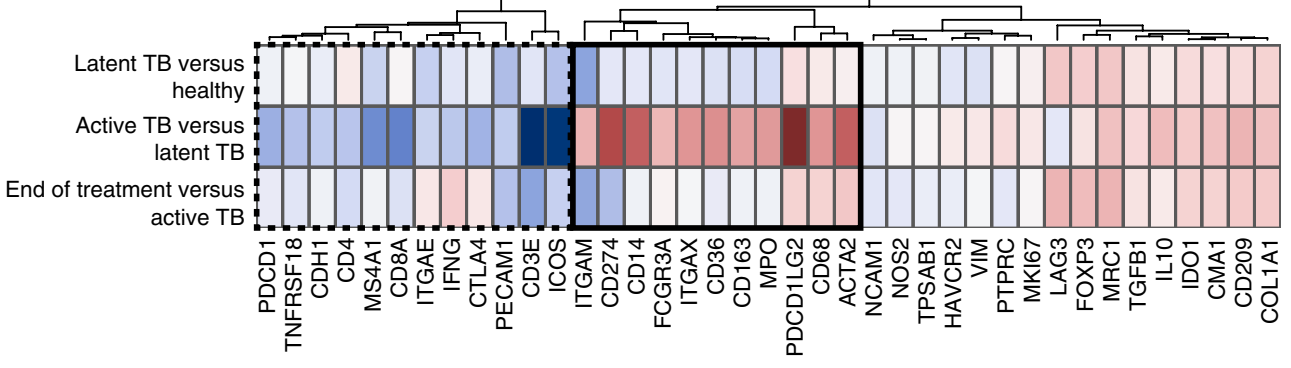

f

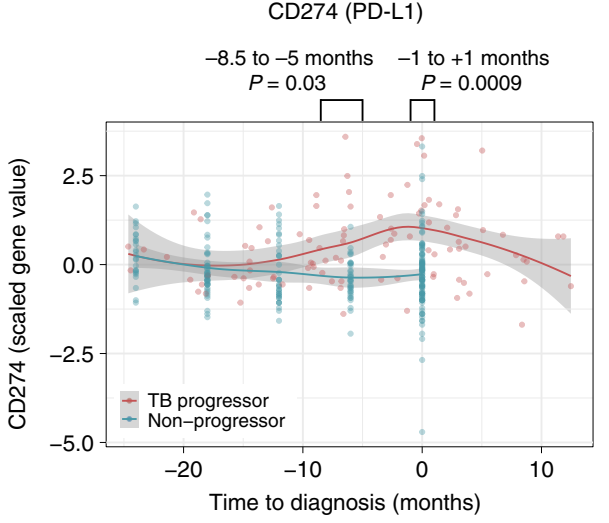

g

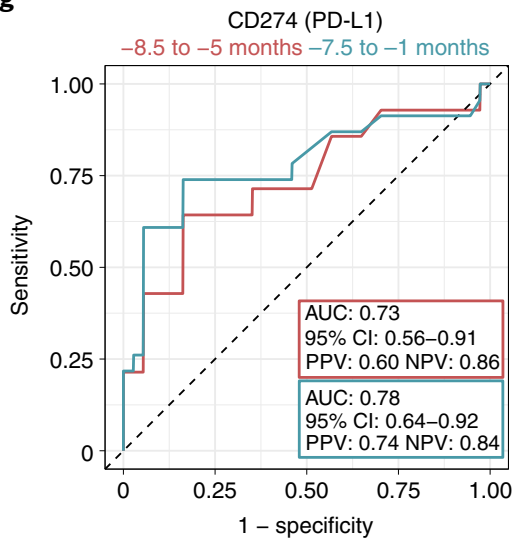

Fig. 6 | Immunoregulatory features of granulomas are reflected in the peripheral blood of TB patients. a, Conceptual overview of the meta-analysis of patients with active TB $(n=647)$ versus healthy controls $(n=197)$. b. Forest plots of gene expression differences in active TB versus healthy individuals. Cohort identifiers are shown on the $y$ axis. Boxes represent the standardized mean difference in gene expression (effect size). The size of the box is proportional to the sample size of that cohort. Whiskers represent the $95 \% \mathrm{Cl}$, and diamonds (black) represent the overall difference in gene expression between two groups by integrating the standardized mean differences across all cohorts. The width of the diamond corresponds to its $95 \% \mathrm{Cl}$. The adjusted $P$ values ( $q$ values, false discovery rate $=5 \%$ ) for the summary effect sizes are shown above each plot. conceptual overview of gene expression analysis across clinical infection stage. $\mathbf{d}$, Heatmap of summary gene expression (mean effect size) values in latent TB $(n=173)$ versus healthy controls $(n=197)$, latent TB $(n=372)$ versus active TB $(n=479)$ and active TB $(n=168)$ versus end of treatment $(n=160)$. Clinical stage is displayed on rows, and genes are displayed across columns hierarchically clustered (Euclidean distance, complete linkage). Genes upregulated in active TB versus latent TB are shown in the solid black box, whereas downregulated genes are in the dashed black box. e, Conceptual overview of the ACS. f, PD-L1 gene expression in the ACS cohort across time prior to and after diagnosis of active TB stratified by progressors (red, $n=34$ ) and nonprogressors (blue, $n=109$ ). Gray silhouette represents the $95 \% \mathrm{Cl}$ of the local polynomial regression (red and blue lines). $P$ values were calculated with a Welch two-sample $t$ test. g, Receiver operating characteristic (ROC) curves of the predictive power of CD274 (PD-L1) expression as a predictor of TB progression. atb, active TB; Itb, latent TB; NPV, negative predictive value; PPV, positive predictive value. 
in expression of PDCD1 (PD-1) or LAG3 (effect size $=-0.41$ and $-0.39, q=0.09$ and 0.05 , respectively).

Next, we analyzed transcriptomic data from 1,549 patients across 24 cohorts to evaluate if these features were specific to active TB (Fig. 6c). In line with our MIBI-TOF analysis, differential expression of genes associated with regulatory myeloid cells (e.g., PD-L1, PD-L2, CD11b, CD11c and CD163) or T cell immune checkpoint (e.g., PD-1 and CTLA4) delineated active from latent infections (Fig. 6d). Moreover, the majority of these genes returned to baseline levels of healthy controls after antimicrobial therapy (Fig. 6d and Extended Data Fig. 8a). Taken together, these results are consistent with a shift toward myeloid-mediated immune regulation that is specific to active TB.

Because PD-L1 gene expression exhibited the largest effect size relative to healthy controls and was upregulated in granulomas, we chose to further understand its relationship with infection dynamics. First, we analyzed the adolescent cohort study (ACS) to determine if PD-L1 expression preceded progression to active disease. Latently infected individuals enrolled in this study underwent regular blood collection and were monitored for symptoms of active infection (Fig. 6e $)^{54,55}$. Within 8.5 to 5 months of clinical diagnosis, PD-L1 transcript levels were significantly elevated in progressors and predictive of progression from latent to active disease (area under the curve $=0.73$, CI 0.56-0.91) (Fig. 6f,g and Extended Data Fig. 8b,c). Strikingly, the predictive performance of PD-L1 in patients 7.5 to 1 months before progression (area under the curve $=0.78$, CI 0.64 0.92 ) was comparable with previously published multigene signatures, despite being a single gene identified by tissue analysis ${ }^{56}$. Taken together, these results support that a shift toward myeloid immune regulation defines the symptomatic stage of TB infection.

We corroborated these results by analyzing the catalysis treatment response cohort to determine if PD-L1 was associated with disease burden and infection clearance (Extended Data Fig. 8d). Patients in this study provided venous blood and underwent PET/ CT imaging upon TB diagnosis ${ }^{57,58}$. PD-L1 expression at diagnosis was directly correlated with total glycolytic activity index, a radiographic metric for lung inflammation (Extended Data Fig. 8e; Pearson $r=0.39, P=4 \times 10^{-4}, t$ test). Twenty-four weeks after treatment, patients were clinically stratified as definitely cured or not cured. Relative to diagnosis, the reduction in PD-L1 expression in patients was two times greater on average in patients who were definitely cured $(n=71)$ than in patients who were not cured $(n=7$; Extended Data Fig. 8f). A nearly identical trend was observed for PD-L2 (PDCDLG2; Extended Data Fig. 8f). In summary, orthogonal analysis of whole blood during TB infection demonstrated synchrony in the local and systemic immune responses during active TB disease.

\section{Discussion}

After nearly 140 years of research into the pathophysiology of human $\mathrm{TB}$, central questions remain unresolved, in part because granuloma formation and progression are difficult to emulate in tractable animal models. Considering this, we developed a framework in which a limited amount of archival tissue and publicly available transcriptomic data were used to identify features of active TB disease in humans. Using clinical specimens from three medical centers, we constructed a spatial cell atlas to relate granuloma structure and composition. We identified 19 cell subsets that organize into eight cellular MEs. TB granulomas appear to follow a consistent structural outline of spatially coordinated PD-L1 and IDO1 and myeloid core-infiltrating $\mathrm{T}_{\text {reg }}$ cells and a striking absence of $\mathrm{T}$ cell activation. Although several of these immune features have been previously identified individually ${ }^{34,45,59,60}$, this study demonstrates an association between myeloid cell regulatory features and reduced lymphocyte activation with spatial and single-cell resolution. Certain features, such as expression of PD-L1 and immunoregulatory macrophages, were present in noninfectious granulomas as well, pointing to universal immune programs associated with granulomatous inflammation. However, compared to other granulomatous conditions, spatially coordinated coexpression of IDO1 and PD-L1 was unique to mycobacterial granulomas.

Granulomas can display a range of disparate outcomes with respect to bacterial burden and inflammatory trajectory ${ }^{10,11}$. The variation in our imaging dataset suggests local outcomes may be driven by unique cellular infiltrate and structure within each granuloma. We observed that features, such as high frequency of $\mathrm{CD}^{+}$ $\mathrm{T}$ cells, corresponded with reduced levels of more differentiated macrophage phenotypes, a profile present in therapeutic resections where PD-L1 and IDO1 expression was diminished. Because CD8 ${ }^{+}$ $\mathrm{T}$ cells are important contributors to TB immunity ${ }^{61,62}$, understanding the immunological environments that promote their activity could reveal novel insights into immune features critical for bacterial clearance.

The high levels of PD-L1 and IDO1 observed in the near absence of PD-1 offers clues into how an immunoregulatory niche during infection is initiated and maintained. We observed that IDO1 and PD-L1 in myeloid cells were colocalized with TGF $\beta$, further confirming the immunoregulatory nature of granuloma macrophages. This is consistent with a TGF- $\beta$ - or IL-10-driven process in which production of these cytokines suppresses inflammation and induces peripheral $\mathrm{T}_{\text {reg }}$ cell activity $28,63,64$. These findings are supported in murine TB, where focal secretion of TGF- $\beta$ within the myeloid core preferentially suppresses neighboring $\mathrm{T}$ cells, and in non-human primates, where granuloma formation associates with IL-10 secretion $^{46,65}$. The next step will be to establish a causal role for TGF- $\beta$ in driving immune suppression in granulomas across the full spectrum of TB.

We next assessed the extent to which the immunoregulatory features identified in archival tissue manifested in peripheral blood. In a multicohort meta-analysis, we identified signatures in blood and analyzed them with respect to disease burden and clinical outcome. As in granulomas, immunoregulatory genes such as PD-L1, IDO1 and CD163 were upregulated in blood. Similarly, genes associated with $\mathrm{T}$ cell activation were downregulated, consistent with the rare incidence of PD-1 or Lag3 in tissue. Importantly, the magnitude of these trends was higher in patients with active disease relative to those with latent or treated infections. PD-L1 displayed the highest effect size of all genes analyzed here. Notably, its expression correlated with pulmonary disease burden and preceded progression to active $\mathrm{TB}$, in line with previous work demonstrating upregulation of $C D 274$ in patients with active $\mathrm{TB}^{66}$. It should be noted that given the low prevalence of monocytes in the blood and prior reports of upregulation of PD-L1 by neutrophils during active TB, it is likely granulocytes drive this systemic gene signature ${ }^{66,67}$.

Both IDO1 and PD-L1 dampen antitumor immune responses in cancer, prompting immunotherapy development ${ }^{68}$. Our findings suggest that similar approaches could be used to block PD-L1-mediated immune suppression in TB. However, evidence of $\mathrm{T}$ cell activation or exhaustion is absent in our dataset and other datasets. Given this, efficacy of PD-L1 blockade could differ substantially from PD-1 blockade. Recent reports of TB reactivation following PD-1 blockade yet fewer instances following PD-L1 blockade illustrate the paradoxical effects that occur with host-directed therapies ${ }^{69-73}$. In our dataset, the small numbers of PD-1 lymphocytes present were largely localized to neighboring TLSs. This raises the possibility that PD-1 blockade exacerbates immunopathology by stimulating TLS-resident and peripheral $\mathrm{T}$ cells while failing to activate granuloma $\mathrm{T}$ cells. This is supported by work in the ultra-low-dose murine model of TB, where a higher proportion of IFN- $\gamma^{+}$-producing, activated $\mathrm{CD} 4^{+} \mathrm{T}$ cells were found at distal lung sites relative to the granuloma ${ }^{46}$. These data emphasize the need to map the temporal and spatial dynamics of these pathways. In line 
with this, a critical next step will be to connect these features to bacterial burden, inflammatory dynamics, intraindividual variability and granuloma age in a primate model that accurately recapitulates human TB pathology.

In conclusion, with our generalizable framework, we identified how cellular composition and immunoregulatory pathways in TB granulomas relate to peripheral immune responses. This has implications for developing host-directed immunotherapies and understanding the immunologic basis of failed immunity in TB. Expression of proteins such as IDO1 and PD-L1 aligns with immune-evasion mechanisms observed in the tumor-immune ME. The interface of granuloma and tumor immunobiology offers new opportunities to explore how tactics of immune evasion in tumors contribute to bacterial persistence in granulomas. Future multiplexed imaging studies of granulomas from controlled TB exposures will offer insight into how these local regulatory dynamics influence granuloma fate and, ultimately, infection outcome.

\section{Online content}

Any methods, additional references, Nature Research reporting summaries, source data, extended data, supplementary information, acknowledgements, peer review information; details of author contributions and competing interests; and statements of data and code availability are available at https://doi.org/10.1038/ s41590-021-01121-x.

Received: 25 May 2021; Accepted: 14 December 2021; Published online: 20 January 2022

\section{References}

1. World Health Organization. Global Tuberculosis Report 2020 https://www. who.int/publications/i/item/9789240013131 (WHO, 2020).

2. Cohen, S. B. et al. Alveolar macrophages provide an early Mycobacterium tuberculosis niche and initiate dissemination. Cell Host Microbe 24, 439-446 (2018)

3. Wolf, A. J. et al. Mycobacterium tuberculosis infects dendritic cells with high frequency and impairs their function in vivo. J. Immunol. 179, 2509-2519 (2007).

4. Bold, T. D. \& Ernst, J. D. Who benefits from granulomas, mycobacteria or host? Cell 136, 17-19 (2009).

5. Davis, J. M. \& Ramakrishnan, L. The role of the granuloma in expansion and dissemination of early tuberculous infectionspan. Cell 136, 37-49 (2009).

6. Ramakrishnan, L. Revisiting the role of the granuloma in tuberculosis. Nat. Rev. Immunol. 12, 352-366 (2012).

7. Cadena, A. et al. Heterogeneity in tuberculosis.Nat. Rev. Immunol. 17, 691-702 (2017).

8. Subbian, S. et al. Lesion-specific immune response in granulomas of patients with pulmonary tuberculosis: a pilot study. PLOS ONE 10, e0132249 (2015)

9. Coleman, M. T. et al. Early changes by (18)fluorodeoxyglucose positron emission tomography coregistered with computed tomography predict outcome after Mycobacterium tuberculosis infection in cynomolgus macaques. Infect. Immun. 82, 2400-2404 (2014).

10. Lin, P. L. et al. Sterilization of granulomas is common in active and latent tuberculosis despite within-host variability in bacterial killing. Nat. Med. 20 75-79 (2013).

11. Martin, C. J. et al. Digitally Barcoding Mycobacterium tuberculosis reveals in vivo infection dynamics in the macaque model of tuberculosis. MBio 8 e00312-e00317 (2017).

12. Carow, B. et al. Spatial and temporal localization of immune transcripts defines hallmarks and diversity in the tuberculosis granuloma. Nat. Commun. 10, 1-15 (2019).

13. Marakalala, M. J. et al. Inflammatory signaling in human tuberculosis granulomas is spatially organized. Nat. Med. 22, 531-538 (2016).

14. Kauffman, K. D. et al. Defective positioning in granulomas but not lung-homing limits CD4 T-cell interactions with Mycobacterium tuberculosis-infected macrophages in rhesus macaques. Mucosal Immunol. 11, 462-473 (2018).

15. Ernst, J. D., Cornelius, A., Desvignes, L., Tavs, J. \& Norris, B. A. Limited antimycobacterial efficacy of epitope peptide administration despite enhanced antigen-specific CD4 T-cell activation. J. Infect. Dis. 218, 1653-1662 (2018)

16. Keren, L. et al. MIBI-TOF: a multiplexed imaging platform relates cellular phenotypes and tissue structure. Sci. Adv. 5, eaax5851 (2019).
17. Krishnan, N., Robertson, B. D. \& Thwaites, G. The mechanisms and consequences of the extra-pulmonary dissemination of Mycobacterium tuberculosis. Tuberculosis 90, 361-366 (2010).

18. Keren, L. et al. A structured tumor-immune microenvironment in triple negative breast cancer revealed by multiplexed ion beam imaging. Cell 174, 1373-1387 (2018).

19. Van Valen, D. A. et al. Deep learning automates the quantitative analysis of individual cells in live-cell imaging experiments. PLoS Comput. Biol. 12, e1005177 (2016)

20. Bannon, D. et al. DeepCell Kiosk: scaling deep learning-enabled cellular image analysis with Kubernetes. Nat. Methods 18, 43-45 (2021).

21. Van Gassen, S. et al. FlowSOM: using self-organizing maps for visualization and interpretation of cytometry data. Cytom. Part A 87, 636-645 (2015).

22. Polena, H. et al. Mycobacterium tuberculosis exploits the formation of new blood vessels for its dissemination. Sci. Rep. 6, 33162 (2016).

23. Oehlers, S. H. et al. Interception of host angiogenic signalling limits mycobacterial growth. Nature 517, 612-615 (2015).

24. Girvan, M. \& Newman, M. E. J. Community structure in social and biological networks. Proc. Natl Acad. Sci. USA 99, 7821-7826 (2002).

25. Chen, Z., Soifer, I., Hilton, H., Keren, L. \& Jojic, V. Modeling multiplexed images with spatial-LDA reveals novel tissue microenvironments. J. Comput. Biol. 27, 1204-1218 (2020)

26. Shi, J. et al. PD-1 controls follicular T helper cell positioning and function. Immunity 49, 264-274 (2018).

27. Ulrichs, T. et al. Human tuberculous granulomas induce peripheral lymphoid follicle-like structures to orchestrate local host defence in the lung. J. Pathol. 204, 217-228 (2004)

28. Difazio, R. M. et al. Active transforming growth factor- $\beta$ is associated with phenotypic changes in granulomas after drug treatment in pulmonary tuberculosis. DARU, J. Pharm. Sci. 24, 6 (2016).

29. Krystel-Whittemore, M. Mast cell: a multi-functional master cell. Front. Oncol. 6, 620 (2016).

30. Gideon, H. P. et al. Single-cell profiling of tuberculosis lung granulomas reveals functional lymphocyte. Preprint at bioRxiv https://doi. org/10.1101/2020.10.24.352492 (2020)

31. Shen, L. et al. PD-1/PD-L pathway inhibits M.tb-specific CD4+ T-cell functions and phagocytosis of macrophages in active tuberculosis. Sci. Rep. 6, 38362 (2016).

32. Mehra, S. et al. Granuloma correlates of protection against tuberculosis and mechanisms of immune modulation by Mycobacterium tuberculosis. J. Infect. Dis. 207, 1115-1127 (2013).

33. Munn, D. H. \& Mellor, A. L. IDO in the tumor microenvironment: inflammation, counter-regulation, and tolerance. Trends Immunol. 37, 193-207 (2016).

34. Gautam, U. S. et al. In vivo inhibition of tryptophan catabolism reorganizes the tuberculoma and augments immune-mediated control of Mycobacterium tuberculosis. Proc. Natl Acad. Sci. USA 115, E62-E71 (2018).

35. Jurado, J. O. et al. Programmed death (PD)-1:PD-ligand 1/PD-ligand 2 pathway inhibits $\mathrm{T}$ cell effector functions during human tuberculosis. J. Immunol. 181, 116-125 (2008).

36. Mulder, K. Cross-tissue single-cell landscape of human monocytes and macrophages in health and disease. Immunity 54, 1883-1900 (2021).

37. Veglia, F., Perego, M. \& Gabrilovich, D. Myeloid-derived suppressor cells coming of age review-article. Nat. Immunol. 19, 108-119 (2018).

38. Gideon, H. P., Phuah, J., Junecko, B. A. \& Mattila, J. T. Neutrophils express pro- and anti-inflammatory cytokines in granulomas from Mycobacterium tuberculosis-infected cynomolgus macaques. Mucosal Immunol. 12, 1370-1381 (2019).

39. Kanamori, M., Nakatsukasa, H., Okada, M., Lu, Q. \& Yoshimura, A. Induced regulatory $\mathrm{T}$ cells: their development, stability, and applications. Trends Immunol. 37, 803-811 (2016).

40. Scott-Browne, J. P. et al. Expansion and function of Foxp3-expressing T regulatory cells during tuberculosis. J. Exp. Med. 204, 2159-2169 (2007).

41. Guyot-Revol, V., Innes, J. A., Hackforth, S., Hinks, T. \& Lalvani, A. Regulatory $\mathrm{T}$ cells are expanded in blood and disease sites in patients with tuberculosis. Am. J. Respir. Crit. Care Med. 173, 803-810 (2006).

42. Green, A. M. et al. CD4 + regulatory T cells in a cynomolgus macaque model of Mycobacterium tuberculosis infection. J. Infect. Dis. 202, 533-541 (2010).

43. Bagaitkar, J. Cellular dynamics of resolving inflammation. Blood 124, 1701-1703 (2014).

44. Wherry, E. J. \& Kurachi, M. Molecular and cellular insights into T cell exhaustion. Nat. Rev. Immunol. 15, 486-499 (2015).

45. Wong, E. A. Low levels of T cell exhaustion in tuberculous lung granulomas. Infect. Immun. 86, e00426-18 (2018).

46. Gern, B. H. et al. TGF $\beta$ restricts expansion, survival, and function of $\mathrm{T}$ cells within the tuberculous granuloma. Cell Host Microbe 29, 594-606 (2021).

47. Baughman, R. P. Sarcoidosis.Lancet 361, 1111-1118 (2003). 
48. Koth, L. L. et al. Sarcoidosis blood transcriptome reflects lung inflammation and overlaps with tuberculosis. Am. J. Respir. Crit. Care Med. 184, 1153-1163 (2011).

49. Maertzdorf, J. et al. Common patterns and disease-related signatures in tuberculosis and sarcoidosis. Proc. Natl Acad. Sci. USA 109, 7853-7858 (2012).

50. Rossi, G. A. et al. Helper T-lymphocytes in pulmonary sarcoidosis: functional analysis of a lung T-cell subpopulation in patients with active disease. Am. Rev. Respir. Dis. 133, 1086-1090 (1986).

51. Facco, M. et al. Sarcoidosis is a Th1/Th17 multisystem disorder. Thorax 66, 144-150 (2011).

52. Haynes, W. A. Empowering multi-cohort gene expression analysis to increase reproducibility. Pac. Symp. Biocomput. 22, 144-153 (2017).

53. Sweeney, T. E., Haynes, W. A., Vallania, F., Ioannidis, J. P. \& Khatri, P. Methods to increase reproducibility in differential gene expression via meta-analysis. Nucleic Acids Res. (2017).

54. Scriba, T. J. et al. Sequential inflammatory processes define human progression from $M$. tuberculosis infection to tuberculosis disease. PLoS Pathog. 13, e1006687 (2017).

55. Zak, D. E. et al. A blood RNA signature for tuberculosis disease risk: a prospective cohort study. Lancet 387, 2312-2322 (2016).

56. Warsinske, H., Vashisht, R. \& Khatri, P. Host-response-based gene signatures for tuberculosis diagnosis: a systematic comparison of 16 signatures. PLoS Med. 16, e1002786 (2019).

57. Roy Chowdhury, R. et al. A multi-cohort study of the immune factors associated with $M$. tuberculosis infection outcomes. Nature 560, 644-648 (2018).

58. Malherbe, S. T. et al. Persisting positron emission tomography lesion activity and Mycobacterium tuberculosis mRNA after tuberculosis cure. Nat. Med. 22, 1094-1100 (2016).

59. Collins, J. M. et al. Tryptophan catabolism reflects disease activity in human tuberculosis. JCI Insight 5, e137131 (2020).

60. Elkington, P. T., Bateman, A. C., Thomas, G. J. \& Ottensmeier, C. H. Implications of tuberculosis reactivation after immune checkpoint inhibition. Am. J. Respiratory Crit. Care Med. 198, 1451-1453 (2018).

61. Flynn, J. L., Goldstein, M. M., Triebold, K. J., Koller, B. \& Bloom, B. R. Major histocompatibility complex class I-restricted $\mathrm{T}$ cells are required for resistance to Mycobacterium tuberculosis infection. Proc. Natl Acad. Sci. USA 89, 12013-12017 (1992)

62. Lalvani, A. et al. Human cytolytic and interferon $\gamma$-secreting CD8 ${ }^{+} \mathrm{T}$ lymphocytes specific for Mycobacterium tuberculosis. Proc. Natl Acad. Sci. USA 95, 270-275 (1998).

63. Li, M. O., Sanjabi, S. \& Flavell, R. A. A. Transforming growth factor- $\beta$ controls development, homeostasis, and tolerance of $\mathrm{T}$ cells by regulatory $\mathrm{T}$ cell-dependent and -independent mechanisms. Immunity 25, 455-471 (2006).
64. Jarnicki, A. G., Lysaght, J., Todryk, S. \& Mills, K. H. G. Suppression of antitumor immunity by IL- 10 and TGF- $\beta$-producing $T$ cells infiltrating the growing tumor: influence of tumor environment on the induction of CD4 + and CD8 + regulatory T cells. J. Immunol. 177, 896-904 (2006).

65. Wong, E. A. et al. IL-10 impairs local immune response in lung granulomas and lymph nodes during early Mycobacterium tuberculosis infection. J. Immunol. 204, 644-659 (2020).

66. McNab, F. W. et al. Programmed death ligand 1 is over-expressed by neutrophils in the blood of patients with active tuberculosis. Eur. J. Immunol. 41, 1941-1947 (2011)

67. Matthew, P. R. Berry et al. An interferon-inducible neutrophil-driven blood transcriptional signature in human tuberculosis. Nature 466, 973-977 (2010).

68. Havel, J. J., Chowell, D. \& Chan, T. A. The evolving landscape of biomarkers for checkpoint inhibitor immunotherapy. Nat. Rev. Cancer 19 , 133-150 (2019)

69. Barber, D. L. et al. Tuberculosis following PD-1 blockade for cancer immunotherapy. Sci. Transl. Med. 11, eaat2702 (2019).

70. Anastasopoulou, A., Ziogas, D. C., Samarkos, M., Kirkwood, J. M. \& Gogas, H. Reactivation of tuberculosis in cancer patients following administration of immune checkpoint inhibitors: current evidence and clinical practice recommendations. J. Immunother. Cancer 7, 239 (2019).

71. Tezera, L. B. et al. Anti-PD-1 immunotherapy leads to tuberculosis reactivation via dysregulation of TNF- $\alpha$. Elife 9, 52668 (2020).

72. Sharpe, A. H., Sher, A., Barber, D. L., Mayer-Barber, K. D. \& Feng, C. G. CD4 $\mathrm{T}$ cells promote rather than control CD4 $\mathrm{T}$ cells promote rather than control tuberculosis in the absence of PD-1-mediated inhibition. J. Immunol. 6, 1598-1607 (2017).

73. Anand, K. et al. Mycobacterial infections due to PD-1 and PD-L1 checkpoint inhibitors. ESMO Open 5, e000866 (2020).

Publisher's note Springer Nature remains neutral with regard to jurisdictional claims in published maps and institutional affiliations.

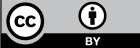

Open Access This article is licensed under a Creative Commons

Attribution 4.0 International License, which permits use, sharing, adaptation, distribution and reproduction in any medium or format, as long as you give appropriate credit to the original author(s) and the source, provide a link to the Creative Commons license, and indicate if changes were made. The images or other third party material in this article are included in the article's Creative Commons license unless indicated otherwise in a credit line to the material. If material is not included in the article's Creative Commons license and your intended use is not permitted by statutory regulation or exceeds the permitted use, you will need to obtain permission directly from the copyright holder. To view a copy of this license, visit http://creativecommons. org/licenses/by/4.0/.

(C) The Author(s) 2022, corrected publication 2022 


\section{Methods}

Human TB granuloma cohort. All human samples were acquired in accordance with institutional review board protocol 46586 ('Generation of an Immune Atlas of Human Tuberculosis Granulomas with Multiplexed Ion Beam Imaging'). Patient consent was not acquired, as only archival clinical specimens were analyzed, with no active participation of humans. FFPE $M t b$-infected tissues were acquired from Stanford Health Care's tissue repository from nine patients undergoing a pretreatment diagnostic biopsy $(n=9)$. Tissues were screened to include those that were positive for acid-fast Bacillus and $M t b$ DNA by polymerase chain reaction. Archival surgical resection tissues were acquired from University of KwaZulu-Natal, Inkosi Albert Luthuli Central Hospital from three patients with $M t b$ infection who underwent therapeutic resection of infected tissue due to infection severity or treatment failure $(n=3)$. Patients with documented HIV coinfection were excluded. Postmortem autopsy specimens were acquired from the University of Texas Health Science Center $(n=3)$. Postmortem diagnosis of $M t b$ infection was confirmed with clinical history, culture, autopsy findings and IHC for $M t b$ antigens. All clinical details for specimens can be found in Extended Data Table 1. Serial sections $(5 \mu \mathrm{m})$ of each specimen were stained with $H \& E$ and inspected by an anatomic pathologist to screen for the presence of granulomatous inflammation. Regions with histologically solid granulomas or cellular granulomatous inflammation surrounding central necrosis were included. Regions with excessive fibrosis or necrosis that were sparsely cellular or acellular were excluded. Two $500 \mu \mathrm{m} \times 500 \mu \mathrm{m}$ FOVs were chosen from each tissue block for imaging. No statistical methods were used to predetermine sample sizes.

Nontuberculous granulomas and controls tissues. Regions of granulomatous inflammation from FFPE sarcoidosis and foreign body reactions from Stanford Health Care were chosen by an anatomic pathologist; $0.5-\mathrm{mm}$ cores were selected and constructed into a tissue microarray. A pulmonary M. avium case was acquired from Stanford Health Care through selection criteria of positive for acid-fast Bacillus staining and polymerase chain reaction positivity for M. avium complex. A $5-\mu \mathrm{m}$ serial section of this specimen was stained with H\&E and inspected by an anatomic pathologist to screen for the presence of granulomatous inflammation. Control tissues of FFPE tonsil, spleen and placenta were acquired from Stanford Health Care. Small regions of each tissue were selected and placed in a tissue microarray.

Incorporation of additional specimens. It should be noted that the original study cohort comprised 20 FOVs across 10 patients, and 10 FOVs from 5 patients were added a later date. Any additional steps taken to process these specimens differently or incorporate them into the dataset are indicated in the relevant sections below.

Antibody preparation. Antibodies were conjugated to isotopic metal reporters as described previously ${ }^{18}$. Following conjugation antibodies were diluted in Candor PBS Antibody Stabilization solution (Candor Bioscience). Antibodies were either stored at $4{ }^{\circ} \mathrm{C}$ or lyophilized in $100 \mathrm{mM} \mathrm{D}-(+)$-Trehalose dehydrate (Sigma-Aldrich) with ultrapure distilled $\mathrm{H}_{2} \mathrm{O}$ for storage at $-20^{\circ} \mathrm{C}$. Before staining, lyophilized antibodies were reconstituted in a buffer of Tris (Thermo Fisher Scientific), sodium azide (Sigma-Aldrich), ultrapure water (Thermo Fisher Scientific) and antibody stabilizer (Candor Bioscience) to a concentration of $0.05 \mathrm{mg} \mathrm{ml}^{-1}$. The antibodies, metal reporters and staining concentrations are listed in Extended Data Table 2. A limitation of this study is that we did not have an antibody for labeling bacteria due to the inherent difficulty of antibody-based detection of $M t b$ in FFPE tissue.

Tissue staining. Tissues were sectioned ( $5 \mu \mathrm{m}$ section thickness) from tissue blocks on gold and tantalum-sputtered microscope slides. Slides were baked at $70{ }^{\circ} \mathrm{C}$ overnight, followed by deparaffinization and rehydration with washes in xylene $(3 \times), 100 \%$ ethanol $(2 \times)$, 95\% ethanol $(2 \times)$, $80 \%$ ethanol $(1 \times)$, $70 \%$ ethanol $(1 \times)$ and $\mathrm{ddH}_{2} \mathrm{O}$ with a Leica ST4020 Linear Stainer (Leica Biosystems). Tissues next underwent antigen retrieval by submerging sides in 3-in-1 Target Retrieval Solution ( $\mathrm{pH} 9$, DAKO Agilent) and incubating at $97^{\circ} \mathrm{C}$ for $40 \mathrm{~min}$ in a Lab Vision PT Module (Thermo Fisher Scientific). After cooling to room temperature, slides were washed in $1 \times$ PBS IHC Washer Buffer with Tween 20 (Cell Marque) with $0.1 \%(\mathrm{w} / \mathrm{v})$ bovine serum albumin (Thermo Fisher). Next, all tissues underwent two rounds of blocking, the first to block endogenous biotin and avidin with an Avidin/Biotin Blocking Kit (BioLegend). Tissues were then washed with wash buffer and blocked for $1 \mathrm{~h}$ at room temperature with $1 \times$ TBS IHC Wash Buffer with Tween 20 with $3 \%$ (v/v) normal donkey serum (Sigma-Aldrich), $0.1 \%(\mathrm{v} / \mathrm{v})$ cold fish skin gelatin (Sigma-Aldrich), $0.1 \%(\mathrm{v} / \mathrm{v})$ Triton X-100, and $0.05 \%(\mathrm{v} / \mathrm{v})$ Sodium Azide. The first antibody cocktail was prepared in 1x TBS IHC Wash Buffer with Tween 20 with $3 \%(\mathrm{v} / \mathrm{v})$ normal donkey serum (Sigma-Aldrich) and filtered through a $0.1-\mu \mathrm{m}$ centrifugal filter (Millipore) prior to incubation with tissue overnight at $4{ }^{\circ} \mathrm{C}$ in a humidity chamber. Following the overnight incubation, slides were washed twice for $5 \mathrm{~min}$ in wash buffer. The second day, an antibody cocktail was prepared as described and incubated with the tissues for $1 \mathrm{~h}$ at $4{ }^{\circ} \mathrm{C}$ in a humidity chamber. Following staining, slides were washed twice for $5 \mathrm{~min}$ in wash buffer and fixed in a solution of $2 \%$ glutaraldehyde (Electron Microscopy
Sciences) solution in low-barium PBS for $5 \mathrm{~min}$. Slides were washed in PBS (1X), $0.1 \mathrm{M}$ Tris at $\mathrm{pH} 8.5(3 \times)$ and $\mathrm{ddH}_{2} \mathrm{O}(2 \times)$ and then dehydrated by washing in $70 \%$ ethanol $(1 \times), 80 \%$ ethanol $(1 \times), 95 \%$ ethanol $(2 \times)$ and $100 \%$ ethanol $(2 \times)$. Slides were dried under vacuum prior to imaging.

MIBI-TOF imaging. Imaging was performed using a MIBI-TOF instrument with a Hyperion ion source. $\mathrm{Xe}^{+}$primary ions were used to sequentially sputter pixels for a given FOV. The following imaging parameters were used: acquisition setting, $80 \mathrm{kHz}$; field size, $500 \mu \mathrm{m} \times 500 \mu \mathrm{m}$ (TB, M. avium and controls) or $450 \mu \mathrm{m} \times 450$ $\mu \mathrm{m}$ (sarcoidosis) at 1,024 $\times 1,024$ pixels; dwell time, $4 \mathrm{~ms}$; median gun current on tissue, $1.45 \mathrm{nA} \mathrm{Xe}^{+}$; ion dose, $3.38 \mathrm{nAmp} \mathrm{h}$ per $\mathrm{mm}^{2}\left(500-\mu \mathrm{m}^{2}\right.$ FOVs) and 3.75 nAmp h per $\mathrm{mm}^{2}\left(450 \mu \mathrm{m}^{2}\right.$ FOVs).

For samples added to the cohort at a later time, the following imaging parameters were used: acquisition setting, $80 \mathrm{kHz}$; field size, $500 \mu \mathrm{m} \times 500 \mu \mathrm{m}$; dwell time, 1-2 ms; median gun current on tissue, $4.36 \mathrm{nA} \mathrm{Xe}^{+}$; ion dose, $2.54 \times$ $5.08 \mathrm{nAmp}$ h per $\mathrm{mm}^{2}$.

Low-level image processing. Multiplexed image sets were extracted, slide background-subtracted, denoised and aggregate filtered as previously described ${ }^{18}$. All parameters for these steps can be found in Extended Data Table 2. In addition to these processing steps, image compensation was performed to account for signal spillover due to adducts and oxides for the following interferences: collagen-1 to IDO1 and Lag3, H3K9Ac to pan-CK and MPO, Chym/Tryp to MPO, Ki-67 to CD209, CD20 to CD16, CD16 to IFN- $\gamma$, CD11c to IDO1 and HLA-DR-DQ-DP to CD11b.

Single-cell segmentation. Nuclear segmentation was performed using an adapted version of DeepCell ${ }^{20}$. DeepCell is a convolutional neural network that can be trained to predict single-cell segmentation across a range of biological platforms. One of the key challenges with segmentation of tissue data is the highly overlapping nature of adjacent nuclei. Previous work found that, although DeepCell is able to generate accurate segmentations using just a nuclear channel, it made errors where the tissue was very densely packed ${ }^{18}$. To improve the accuracy on these densely packed cells, especially immune cells, a modified version of the network was trained which included both a nuclear channel and a membrane channel as the basis for prediction.

First, training data were generating by manually annotating all of the nuclei in five separate images taken from a MIBI-TOF study of patients with melanoma, where each image was stained with $\mathrm{HH} 3$ to identify nuclei and $\mathrm{Na}^{+} \mathrm{K}^{+} \mathrm{ATPase}$ to identify the cell membrane (Extended Data Fig. 1d). For each image, the ImageJ platform was used to identify the location of each unique nuclei, which was then used to generate three distinct masks: one representing the interior of each nuclei, one representing the border of each nuclei and one representing the background of the image (which is all pixels not belonging to the first two classes). These three masks were created for each of the five images.

These masks, along with the image data, were used to train DeepCell. Each image was divided into overlapping crops of $64 \times 64$ pixels. Each crop was randomly flipped, rotated and sheared during training to further augment the available data. Stochastic gradient descent was used to train the network, with the performance assessed on a held-out portion of the data not seen by the network during training. This was combined with early stopping to prevent overfitting to the training data.

The network was trained to the predict which of the three classes each pixel in an image belongs to. The output of the network was a probability mask representing the confidence of the network in assigning each pixel to one of the three classes. All MIBI-TOF images from our cohort were provided as input to the network, with $\mathrm{HH} 3$ as the nuclear channel and CD45 as the membrane channel. To generate single-cell segments, the probability mask for the nuclear interior was thresholded, smoothed and run through the watershed algorithm as previously described ${ }^{18}$. Post-processing parameters such as the background threshold and smooth value were manually defined to balance the tradeoff between oversegmenting and undersegmenting the cells. Finally, we applied a three-pixel radial expansion around each nucleus to define the cell object boundaries.

A correction was applied to FOVs that contained MNGCs. Each MNGC was identified using a combination of HH3, CD45 and Vimentin and manually segmented in ImageJ to produce a mask of each MNGC. All pixels within the binary mask were reassigned to belong to the MNGC cell object(s). In total, 15 of 30 regions imaged contained MNGCs, with the number of MNGCs per field ranging from 1 to 9 .

Single-cell phenotyping and composition. Single-cell data were extracted for all cell objects and area normalized. Cells with a sum of $<0.1$ area-normalized counts across all lineage channels were excluded from analysis. Single-cell data were linearly scaled with a scaling factor of 100 and asinh-transformed with a cofactor of 5. All mass channels were scaled to the 99.9th percentile. In order to assign each cell to a lineage, the FlowSOM clustering algorithm was used in iterative rounds with the Bioconductor 'FlowSOM' package in $\mathrm{R}$ (ref. ${ }^{21}$ ). The first clustering round separated cells into four major lineages using the 'Metaclustering_consensus' function (immune, epithelial, fibroblast and endothelial). Immune cells were 
then clustered again to delineate $\mathrm{B}$ cells, $\mathrm{CD} 4^{+} \mathrm{T}$ cells, $\mathrm{CD} 8^{+} \mathrm{T}$ cells, $\mathrm{T}_{\text {reg }}$ cells, neutrophils, mast cells and mononuclear phagocytes (macrophages, monocytes and DCs). Immune cells with an expression profile not consistent with any of those subsets were annotated as 'other immune.' Lastly, the mononuclear phagocytes were clustered to 25 metaclusters that were merged into 7 groups. Giant cells were manually identified. $\gamma \delta \mathrm{T}$ cells were annotated as T cells with CD3 signal greater than or equal to the mean expression of $C D 4^{+} \mathrm{T}$ cells and TCR- $\delta$ signal $>0.5$ normalized expression. CD163 macrophages were identified as those myeloid cells with CD163 signal >0.5 normalized expression. Criteria used for assigning cell phenotypes can be found in Extended Data Table 3. Single-cell data that were collected for samples added to the cohort at a later date were extracted and normalized as described above, with 99.9th-percentile scaling done separately for the batch. In order to assign each cell to a lineage, the FlowSOM clustering algorithm was used in iterative rounds, and each resulting cluster was manually mapped to one of the original 20 phenotypic clusters. CD $209^{+} \mathrm{DCs}, \mathrm{CD} 14^{+} \mathrm{CD} 16^{+}$ monocytes and epithelial cells were not annotated in samples 4-6 and 15 due to exclusion of CD209, CD16 and pan-Keratin in the panel used to stained these additional specimens. The relative abundance of all major lineages was calculated out of total cells per FOV, and the relative frequency of immune cell subsets was calculated out of total immune cells per FOV.

Protein enrichment analysis. A spatial enrichment approached was used as previously described ${ }^{18,74}$ to identify patterns of protein enrichment or exclusion across all protein pairs in all samples. $\mathrm{HH} 3, \mathrm{Na}^{+} \mathrm{K}^{+}$ATPase and HLA class 1 were excluded from the analysis. For each pair of markers, $\mathrm{X}$ and $\mathrm{Y}$, the number of times cells positive for protein $\mathrm{X}$ was within a $\sim 50-\mu \mathrm{m}$ radius of cells positive for protein $Y$ was counted. Thresholds for positivity were customized to each marker individually using a silhouette scanning approach from the MetaCyto software in $\mathrm{R}$ (ref. ${ }^{75}$ ). Thresholds were validated both by visual inspection of positive and negative cells in image sets and by inspection of the threshold overlaid on a histogram of signal distribution in single cells per marker (Extended Data Fig. 1e). A null distribution was produced by performing 1,000 bootstrap permutations where the locations of cells positive for protein $\mathrm{X}$ and $\mathrm{Y}$ were randomized. A $\mathrm{z}$-score was calculated comparing the number of true cooccurrences of cells positive for protein $\mathrm{X}$ and $\mathrm{Y}$ relative to the null distribution. For each pair of proteins, $\mathrm{X}$ and $\mathrm{Y}$, the average $\mathrm{Z}$-score was calculated across all TB FOVs. Next, all positive enrichments between protein pairs (average $\mathrm{z}$-score $>0$, excluded self-self protein enrichment scores) were used to produce a weighted, undirected network, where the nodes are the individual markers and the edge weights are proportional to the average $\mathrm{z}$-score (where a higher $\mathrm{z}$-score indicates a shorter edge length). The leading eigenvector algorithm for community detection was used to identify protein enrichment communities in this network ${ }^{76}$.

Spatial-LDA. Spatial-LDA is an adaptation of LDA, a machine-learning approach used to model topics in text documents, where topics consist of words with a high probability of co-occurrence, allowing mapping of topics to abstract definitions (e.g., ['cat', 'frog', 'horse] $\rightarrow$ 'animals'). Spatial-LDA builds on this paradigm by representing CPMs as documents and cell types as words. Spatial-LDA was conducted to identify topics (here referred to as MEs) across 26 TB FOVs. This included 20 FOVs from 10 patients in the current cohort along with 6 FOVs from 3 patients that were excluded from later analysis due to presence of HIV coinfection. All input data for producing the spatial-LDA model can be found in the data and code availability sections. Cell types with fewer than 100 cells across the entire cohort were excluded from analysis ( $\gamma \delta$ T cells and CD209+ DCs). Furthermore, MNGCs were excluded due to their cell size. Spatial-LDA was implemented as described previously ${ }^{25}$, with $d=1,000$, a spatial radius $r=50 \mu \mathrm{m}$ to complement the protein enrichment analysis and an ME number of $n=8$. The ME number was determined empirically. For each FOV, a MaxPM was produced by classifying each cell to the ME with the highest probability and coloring that cell by its ME and probability. The 10 FOVs added at a later date were not included in generation of the spatial-LDA model but were assigned topic probabilities based on the same model built on the original 26 FOVs using the same cell types and spatial parameters as input. The cell type preferences for each $\mathrm{ME}$ were defined by assessing the mean probability for all cell types across all MEs. The mean expression for each functional marker across MEs was calculated by weighting protein expression by ME probability and calculating the mean of weighted expression values across markers and MEs. To cluster FOVs based on ME profile, the Pearson correlation coefficient was calculated between all pairs of TB FOVs based on the relative frequency of cells in each ME. The coefficients were used to hierarchically cluster the FOVs using complete linkage and a distance metric of 1 - correlation coefficient. To identify consensus clusters, the percent variance explained was measured across a range of 1-10 clusters. The elbow point of this plot was used to select the optimal number of clusters to capture the maximal variance in our dataset.

Identification of the myeloid core. In order to assess which MEs represented the histologically defined myeloid core, binary masks of the myeloid core were generated for 15 FOVs. The masks were generated by first combining the signal of CD11c, CD11b, CD14, CD206, CD68 and PD-L1. The combined images were imported into ImageJ and hand-annotated using ROI annotation tools. The annotated ROI was exported as a binary mask. This mask was further processed in MATLAB to close any holes, exclude objects smaller than 1,000 pixels and dilate the mask to smooth edges. Next, cells were assigned to belonging to the myeloid core if they had complete overlap with the binary mask. Cells on the mask boundary or outside of the mask were designated as 'nonmyeloid core.' The proportion of cells in the myeloid core was assessed across each ME for the 15 FOVs and MEs with a median frequency in the myeloid core $>50 \%$ were designated as myeloid core MEs.

Myeloid cell UMAP visualization. UMAP embeddings were determined for all myeloid cells using the $\mathrm{R}$ implementation ${ }^{77}$ with the parameters $\mathrm{n}$-neighbors = 15 and min_dist $=0.5$ and the following markers: CD45, CD68, CD206, CD11c, CD11b, CD14, CD16, CD209, CD163, MPO/calprotectin and mast cell chymase/ tryptase.

Immunoregulatory protein analysis. Positivity thresholds for IDO1, PD-L1, PD-1 and Lag3 were automatically identified as described above. Immune control tissues tonsil, spleen and placenta were used to validate antibody performance. Correlation between IDO1 and PD-L1 was analyzed across the entire cohort in myeloid cells using Pearson correlation analysis. The frequencies of cells positive for IDO1 and PD-L1 were enumerated across all subsets. To assess PD-L1 and IDO1 positivity with respect to ME and cell subset, the total number of cells across all myeloid subsets per ME was pooled across all FOVs. The quantity of cells for each subset positive for IDO1 or PD-L1 was enumerated per ME. Any ME with $<1 \%$ of the total for a subset was excluded from analysis. PD- 1 and Lag3 expression was analyzed on lymphocytes or total immune cells. PD-1 and Lag3 expression was also analyzed on immune cells from a human TNBC cohort that was previously reported by our group ${ }^{18}$. Positivity for PD-1 and Lag3 for TNBC immune cells was defined as described in the original study (normalized signal $>0.5$ ).

Regression analysis. In Fig. $1 \mathrm{i}$ and Extended Data Fig. 4h, linear regression was performed using the $\operatorname{lm}()$ command in R. The quality of the fit was evaluated and reported with the $r^{2}$, Pearson $R$, and $P$ value as measured by a two-tailed $t$ test.

ISH. ISH studies were performed with the commercially available RNAscope HD Reagent Kit (Advanced Cell Diagnostic) for single-plex chromogenic ISH. Following this protocol, FFPE tissue sections were baked, deparaffinized and incubated with RNAscope Hydrogen Peroxide solution for $10 \mathrm{~min}$ at room temperature. Antigen retrieval was carried out in $1 \times$ RNAscope Target retrieval solution for $30 \mathrm{~min}$ at $99^{\circ} \mathrm{C}$ followed by protease digestion for $30 \mathrm{~min}$ at $40^{\circ} \mathrm{C}$ in the HybEZ oven (Advanced Cell Diagnostic). All granuloma tissue along with human spleen and melanoma was probed for human IFNG, human TGFB1, positive control probe human $U B C$ and negative control probe bacterial DapB. Additionally, commercially available HeLa cell pellet control slides were stained for $U B C$ and $D a p B$. Target probes were hybridized for $2 \mathrm{~h}$ at $40^{\circ} \mathrm{C}$ using the HybEZ oven, followed by a series of six amplification steps. Following amplification, slides were stained using a chromogenic substrate (fast red) and counterstained using 50\% Gill's hematoxylin (American MasterTech) before evaluation by light microscopy, where each RNA transcript appeared as a distinct dot of red chromogen. QuPath software was used for quantification of results ${ }^{78}$. Briefly, each image was annotated to include only granulomatous inflammation and exclude surrounding tissue where present. Necrotic regions were also excluded. QuPath cell detection was used to detect cell objects in each image via the hematoxylin channel. Next subcellular detection was run on the fast-red channel with parameters set to include spot clusters. Total estimated spots, cell object counts and region areas were exported and analyzed in R. To separately analyze the myeloid core and lymphocytic zones of the granuloma, individual regions representing each histological zone were annotated based off the hematoxylin channel per image, and the signal was quantified as described previously.

Cell composition analysis of sarcoidosis and TB. Single cells from sarcoidosis FOVs were segmented as described above. Single-cell data were extracted, transformed and normalized along with TB single-cell data. Single cells were included in the described FlowSOM clustering procedure.

IHC of PD-L1 and IDO1. IHC for PD-L1 and IDO1 was performed using the antibody reagents listed in Extended Data Table 2 at a concentration of $1 \mu \mathrm{g} \mathrm{ml}^{-1}$. The IHC protocol mirrors the MIBI-TOF protocol, with the addition of blocking endogenous peroxidase activity with $3 \% \mathrm{H}_{2} \mathrm{O}_{2}$ (Sigma-Aldrich) in $\mathrm{ddH}_{2} \mathrm{O}$ after epitope retrieval. On the second day of staining, instead of proceeding with the MIBI-TOF protocol, tissues were washed twice for $5 \mathrm{~min}$ in wash buffer and stained using ImmPRESS universal (Anti-Mouse/Anti-Rabbit) kit (Vector Laboratories).

Whole-blood transcriptomic analysis. Publicly available gene expression datasets (Extended Data Table 4) were collected, annotated and used for meta-analysis conducted using MetaIntegrator ${ }^{52}$. Gene expression matrices were prepared for each dataset to determine effect sizes for genes of all proteins included in the 
MIBI-TOF analysis and an additional set of genes with similar biological function, such as ICOS and CTLA4 (Extended Data Table 5). Summary effect sizes were calculated to assess gene expression differences across clinical groups (healthy, active TB, latent TB, end of treatment, TB progression and during treatment). For the catalysis treatment response cohort gene expression measurements at diagnosis of TB were correlated with matched total glycolytic activity index, a readout of PET-CT activity. A linear regression was fit between CD274 gene expression and TGAI and the correlation was assessed with Pearson correlation analysis. To assess CD274 and PDCDLG2 gene expression overtreatment, expression values were normalized to the measurement taken at diagnosis (day 0 ). Gene expression data in the ACS were separated by progression status. Local regression was used to fit the gene expression data over time in each group. The significance of separation between progressors and non-progressors was calculated in two different time intervals using a Student's t-test. We selected time points for this analysis by looking at the peak of separation $(-1$ to +1 months) and the earliest time point where we observed substantial separation between groups (i.e., where the error regions were not overlapping), which was around -6 months. The time interval was extended to capture a sufficient number of progressors around the -6-month time point to power the analysis. CD274 expression was also compared in a 'before/after' analysis with the baseline measurement reflecting the earliest measurement prior to diagnosis and the diagnosis measurement representing the closest measurement taken to diagnosis with active TB (within 30 days before or after diagnosis). The predictive power of CD274 expression was evaluated using ROC analysis. Negative and positive predicted values represent the proportion of samples that were actually negative/positive over the number of negative/positive samples reported by setting the threshold using Youden's method.

Statistics and reproducibility. All FOVs were included in all analyses. Data collection and analysis were not performed blind to the type of specimen. Processed images were displayed to show representative imaging data, but quantification of results was performed on unaltered images. For significance testing of cell, phenotypic and ME data, we conservatively applied tests that did not assume normality. $t$ tests were applied for linear regression and correlation analyses in line with the assumption that errors and residuals are normally distributed. Statistical analysis of the transcriptomic data employed multiple hypothesis correction and significance testing as previously established ${ }^{57}$.

Software. Image processing was conducted with MATLAB 2016a and MATLAB 2019b. Statistical analysis was conducted in MATLAB 2016a, MATLAB 2019b and R v3.6.2. Python 3.6 was used for implementation of spatial-LDA. Data visualization and plots were generated in R. Representative images were processed in Adobe Photoshop, and figures were prepared in Adobe Illustrator. Schematic visualizations were produced at https://biorender.com.

Reporting Summary. Further information on research design is available in the Nature Research Reporting Summary linked to this article.

\section{Data availability}

All images and annotated single-cell data are deposited in Mendeley's data repository and can be accessed using the following link: https://doi.org/10.17632/dr5fkgtrb6.

\section{Code availability}

All custom code used to analyze data has been deposited in GitHub and can be accessed using the following link: https://github.com/angelolab/publications/tree/ master/2022-McCaffrey_etal_HumanTB.

\section{References}

74. Hartmann, F. J., et al. Multiplexed single-cell metabolic profiles organize the spectrum of cytotoxic human T cells. Preprint at bioRxiv https://doi org/10.1101/2020.01.17.909796 (2020).

75. Hu, Z. et al. MetaCyto: a tool for automated meta-analysis of mass and flow cytometry data. Cell Rep. 24, 1377-1388 (2018).
76. Newman, M. E. J. Finding community structure in networks using the eigenvectors of matrices. Phys. Rev. E 74, 036104 (2006).

77. McInnes, L., Healy, J. \& Melville, J. UMAP: uniform manifold approximation and projection for dimension reduction. Preprint at https://arxiv.org/ abs/1802.03426 (2018).

78. Bankhead, P. et al. QuPath: open source software for digital pathology image analysis. Sci. Rep. 2017, 16878 (2017).

\section{Acknowledgements}

We thank T. Risom, D. Glass, M. Carter, J. Mattila, J. Flynn and A. Kasmar for discussions and comments. We thank P. Chu and the Stanford Human Histology Core for providing technical assistance. E.F.M. was supported by the National Science Foundation (graduate research fellowship grant 2017242837) and training grant 5T32AI007290. L.K. was a Damon Runyon Fellow supported by the Damon Runyon Cancer Research Foundation (DRG-2292-17) and a nonstipendiary awardee of the EMBO long-term fellowship (ALTF 1128-2016). N.F.G. was supported by the National Cancer Institute (grant CA246880-01) and Stanford University (graduate fellowship). A.J.C.S. was supported by the National Institutes of Health (grants R61/33AI138280 and R01AI134810), CRDF Global, the South African Medical Research Council and a National Research Foundation BRICS multilateral grant. P.K. was funded in part by the Bill and Melinda Gates Foundation (OPP1113682), the National Institute of Allergy and Infectious Diseases (grants 1U19AI109662, U19AI057229 and 5R01AI125197), the Department of Defense (contracts W81XWH-18-1-0253 and W81XWH1910235) and the Ralph \& Marian Falk Medical Research Trust. M.A. was supported by the National Institutes of Health (grants 5U54CA20997105, 5DP5OD01982205, 1R01CA24063801A1, 5R01AG06827902, 5UH3CA24663303, 5R01CA22952904, 1U24CA22430901, 5R01AG05791504 and 5R01AG05628705), the Department of Defense (contracts W81XWH2110143), the Wellcome Trust and other funding from the Bill and Melinda Gates Foundation, Cancer Research Institute, the Parker Center for Cancer Immunotherapy and the Breast Cancer Research Foundation.

\section{Author contributions}

E.F.M. conceived the study design, performed experiments, analyzed data, and wrote the manuscript. M.D. and S.G. conducted the blood transcriptomics analysis. L.K. assisted with analysis conceptualization. Z.C. and V.J. implemented the spatial-LDA analysis. L.K., A.D., N.F.G., A.B. and W.G. assisted with data analysis. R.K. assisted with ISH analysis. A.D., M.B. and C.C. assisted with assay development. D.V.V. developed DeepCell. D. Kalman, D. Kaushal, S.A.K. and S.M. assisted with study and analysis conceptualization. M.F., P.K.R., E.F., M.V.D.R., N.B., R.L.H. and A.J.C.S. provided human samples and consulted on tissue cohort design. S.C.B., P.K. and M.A. supervised the work.

\section{Competing interests}

M.A. and S.C.B. are inventors on patent US20150287578A1, which covers the mass spectrometry approach utilized by MIBI-TOF to detect elemental reporters in tissue using secondary ion mass spectrometry. M.A. and S.C.B. are board members and shareholders in IonPath, which develops and manufactures the commercial MIBI-TOF platform. E.F.M. has previously consulted for IonPath. The remaining authors declare no competing interests.

\section{Additional information}

Extended data is available for this paper at https://doi.org/10.1038/s41590-021-01121-x. Supplementary information The online version contains supplementary material available at https://doi.org/10.1038/s41590-021-01121-x.

Correspondence and requests for materials should be addressed to Michael Angelo.

Peer review information Nature Immunology thanks Joel Ernst and the other, anonymous, reviewer(s) for their contribution to the peer review of this work $\mathrm{N}$. Bernard was the primary editor on this article and managed its editorial process and peer review in collaboration with the rest of the editorial team.

Reprints and permissions information is available at www.nature.com/reprints. 
a Approximate H\&E (serial section) Pulmonary Resection: Patients 1-3 Pulmonary Post-mortem: Patients 4-6 Pulmonary Biopsy: Patients 7,8 Extrapulmonary Biopsy: Patients 9-15

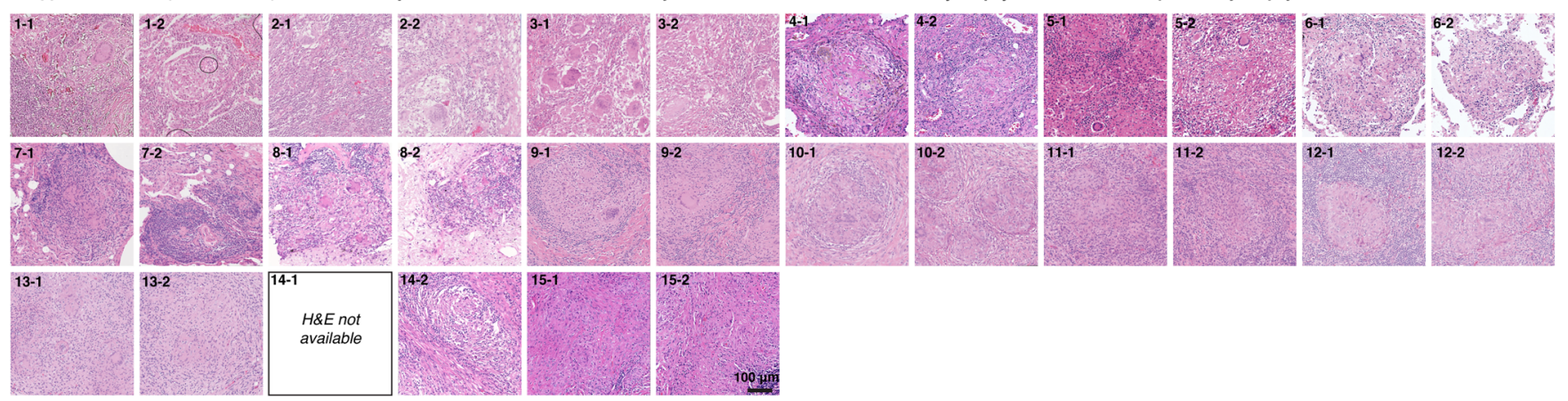

b Antibody Panel

c Antibody Panel-Immune Controls
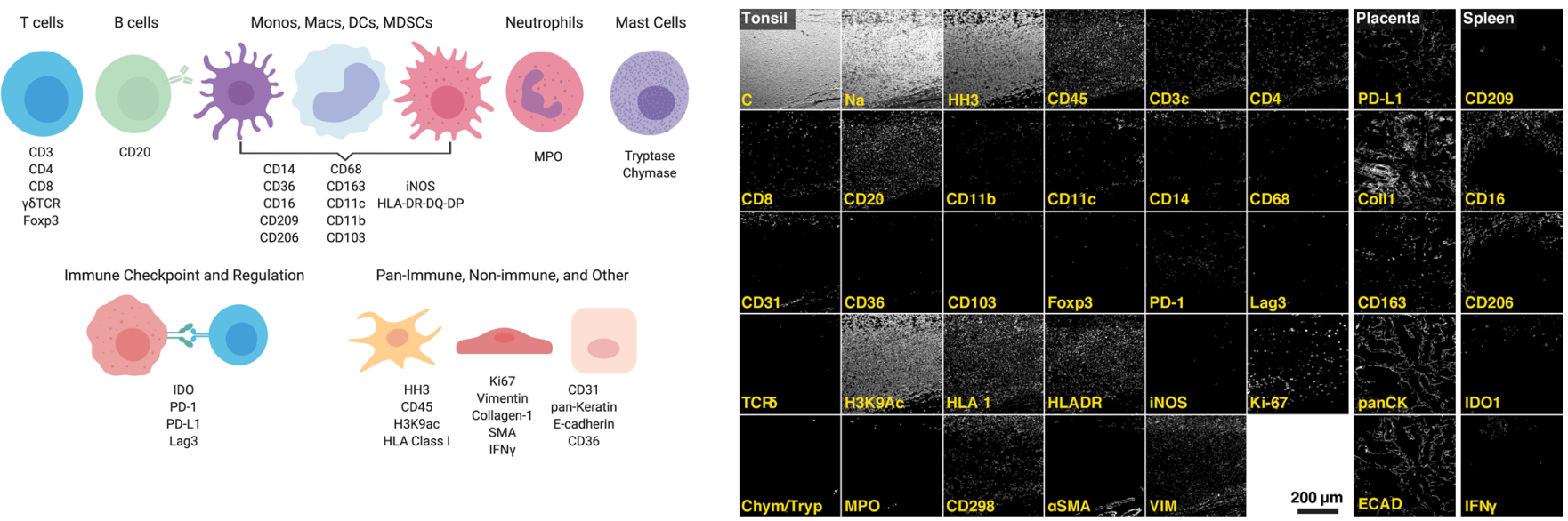

d Segmentation Overview

e Automatic Marker Threshold Detection

1. Train Deepcell CNN: Membrane-based Nuclear Segmentation

Training Data: Melanoma Manually Segmented
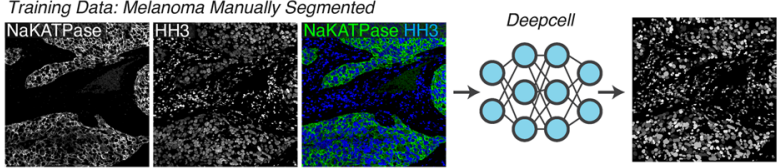

2. Run model on granuloma data

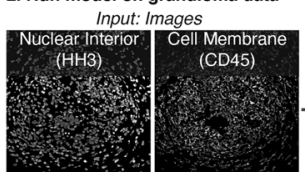

Output: Pixel Classification (Probability Maps)

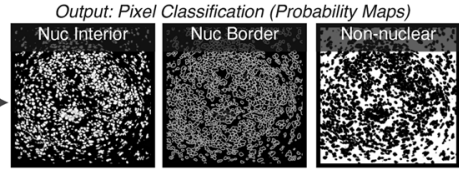

3. Post-processing
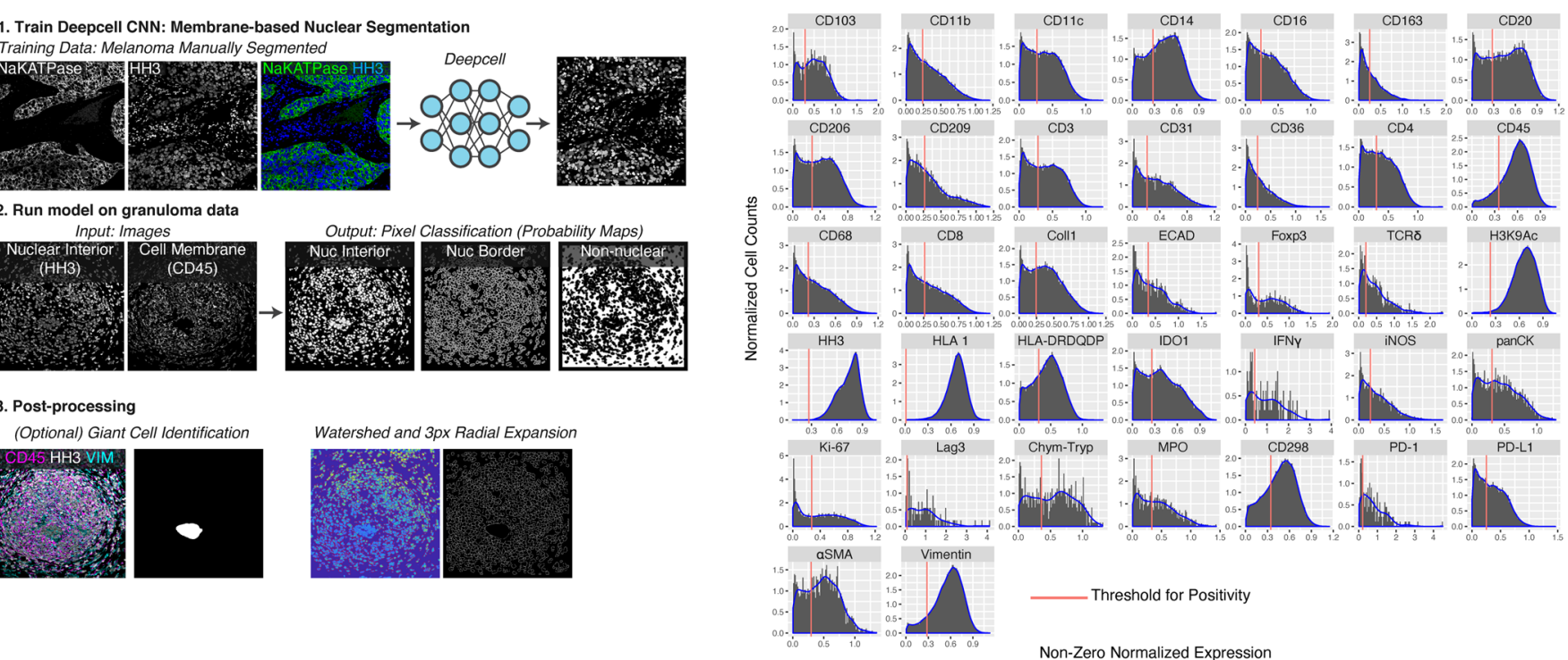

Extended Data Fig. 1 | Multiplexed imaging of human TB granulomas. a, Hematoxylin and eosin-stained serial sections of FOVs for MIBI-TOF imaging. b. Multiplexed antibody panel grouped by marker category. c, Grayscale images of endogenous ion signal and proteins in control tissues (tonsil, spleen, placenta). d, Workflow for Deepcell-based segmentation of single cells from multiplexed images. e, Histograms of non-zero signal for all proteins from single-cell data. Blue line represents Gaussian smoothed density fit of histogram. Red line represents automatically identified threshold for marker positivity. 
a FlowSOM Clustering Procedure

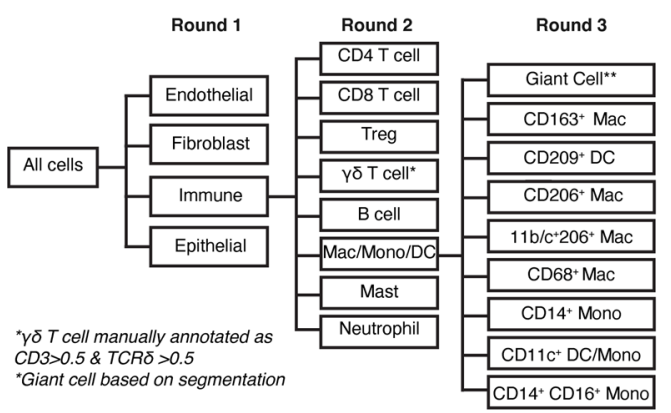

b

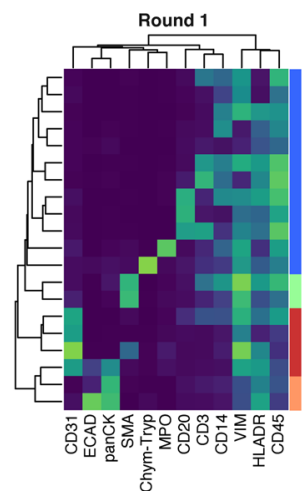

Mean Normalized Expression

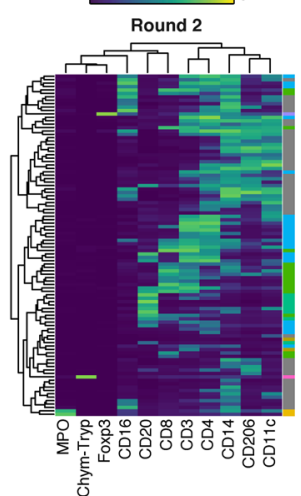

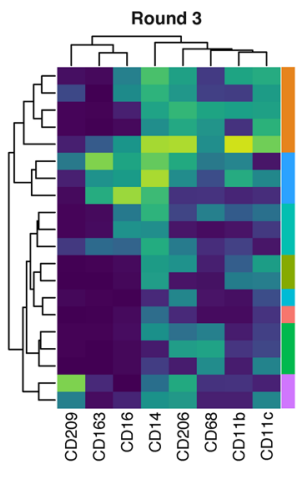

Immune Endothelial CD4 T cell Treg Other Immune Mast Cell 11b/c+206+ Mac CD14+CD16 Mono CD206 Mac CD68 $^{+}$Mac

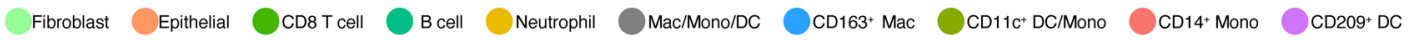
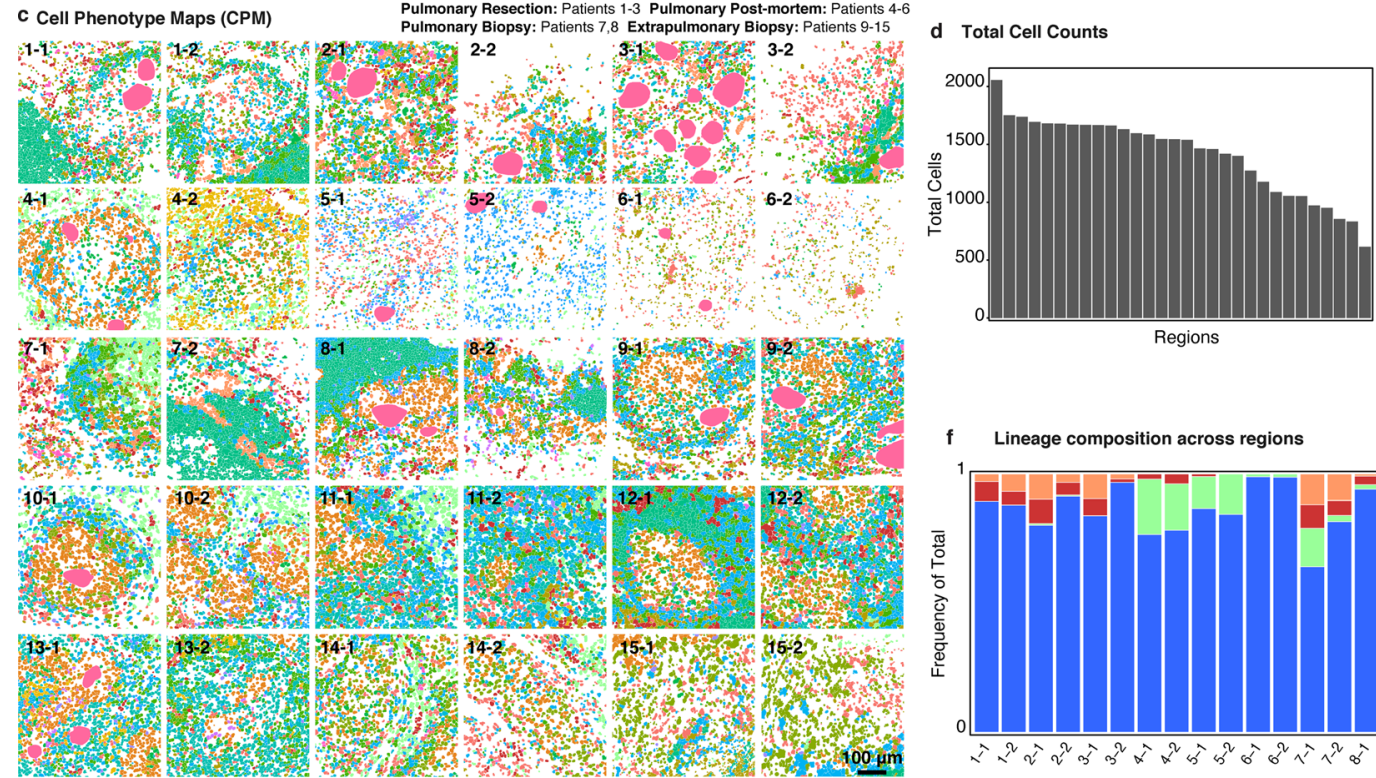

e Bulk lineage composition
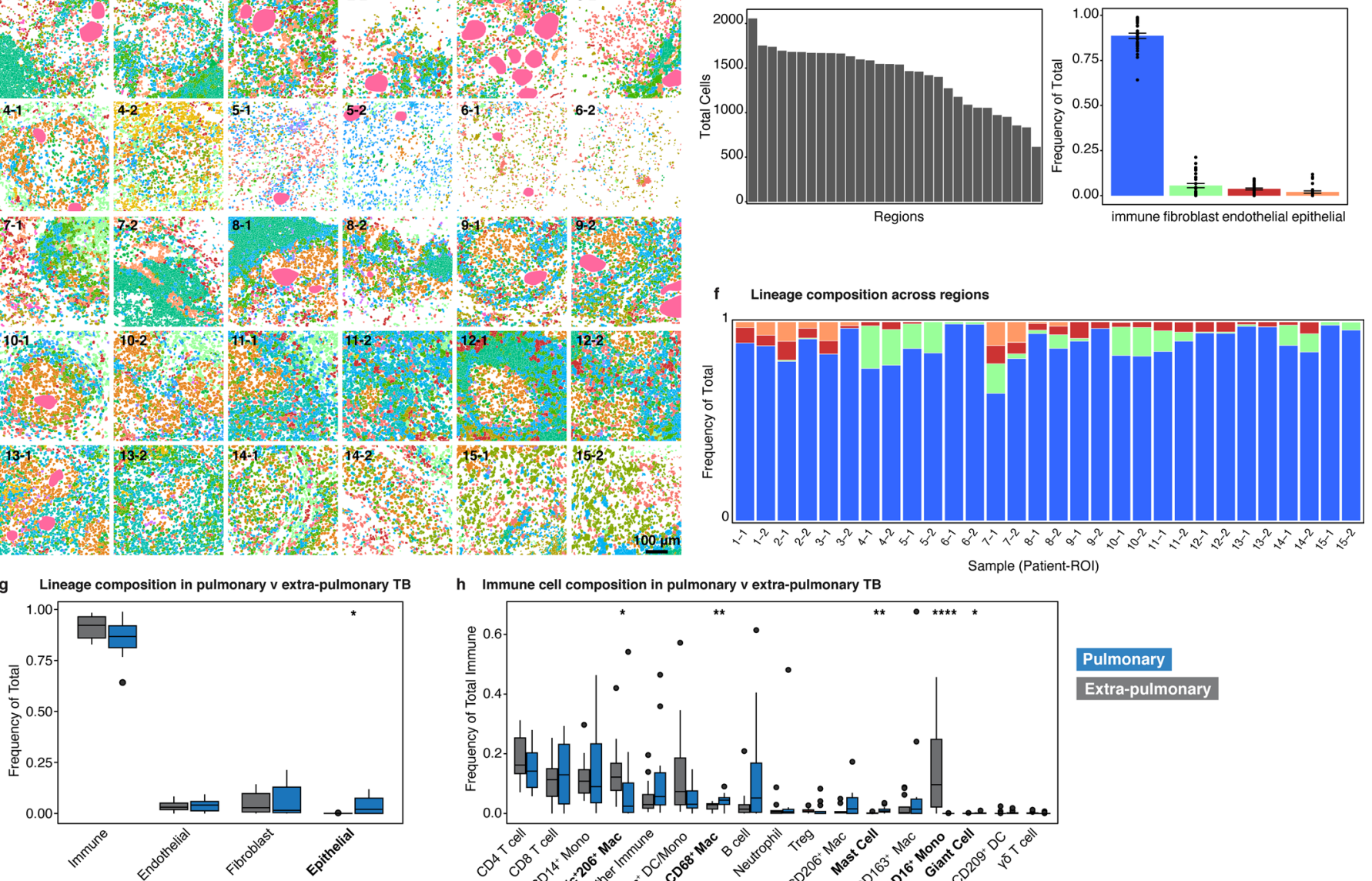

h Immune cell composition in pulmonary v extra-pulmonary $T B$

Sample (Patient-ROI)

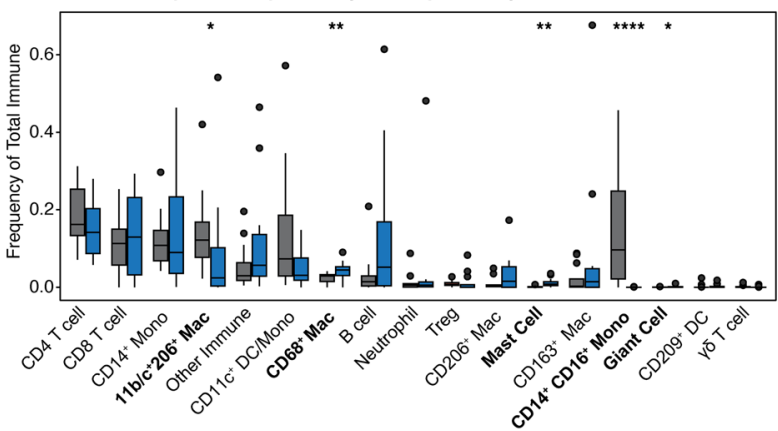

Pulmonary

Extra-pulmonary

CD4 T cell \& CD8 T cell count across specimen groups
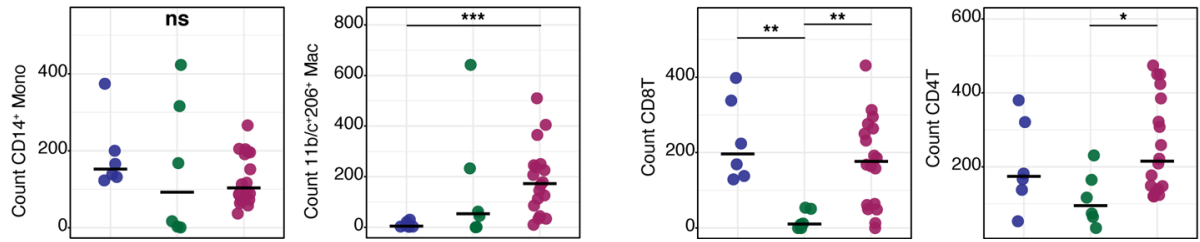

Resection Post-mortem Blopsy

Extended Data Fig. 2 | See next page for caption. 
Extended Data Fig. 2 | Single-cell phenotypic composition of human TB granulomas. a, Conceptual overview of hierarchical FlowSOM algorithm application. $\mathbf{b}$, Heatmap of cell lineages clustered by mean normalized protein expression of markers shown along columns. c, Cell phenotype maps for all FOVs. d, Total cell counts across all FOVs sorted by descending order. e, Major cell lineage composition across all FOVs. Bars represent mean \pm SEM $(n=30)$. f, Major cell lineage frequency of total cells broken down by FOV. $\mathbf{g}$, Frequency of major lineages in pulmonary (blue) versus extrapulmonary (grey) TB granulomas. h, Frequency of immune cell subsets (of total immune cells) in pulmonary (blue) versus extrapulmonary (grey) TB granulomas. $\mathbf{i}$, Count of CD14+ monocytes and $11 \mathrm{~b} / \mathrm{c}^{+} 206^{+}$macrophages cells colored by specimen origin. Line represents the median. $\mathbf{j}$, Count of CD4+ and CD8 ${ }^{+} \mathrm{T}$ cells colored by specimen origin. Line represents the median. Boxplots display the median and interquartile range (IQR, 25-75\%) with whiskers representing the upper- and lower-quartile $\pm 1.5^{\star} \mathrm{IQR}$. P-values were determined with a Wilcoxon Rank Sum Test (two-tailed) where: ${ }^{\star} p<0.05,{ }^{\star \star} p<0.01,{ }^{\star \star \star} p<0.001$. 


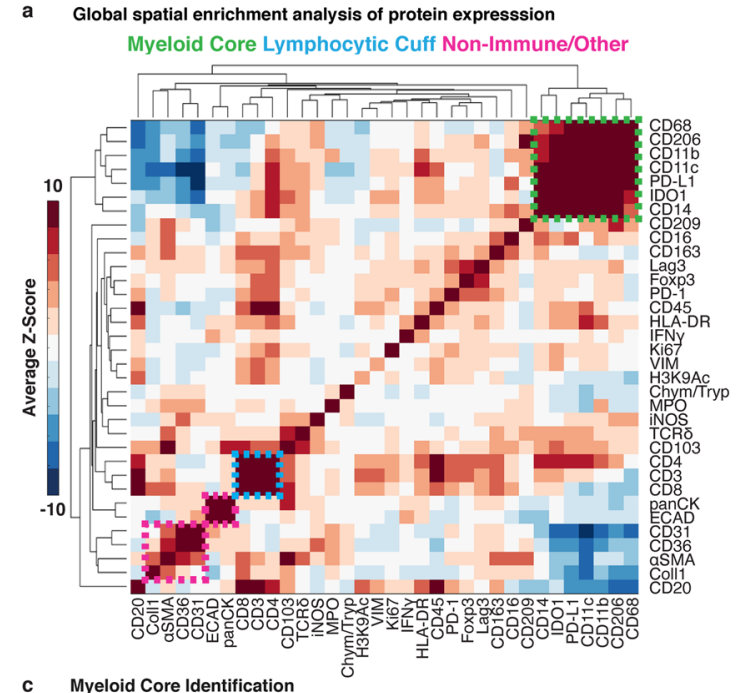

c Myeloid Core Identification

Myeloid core manually gated

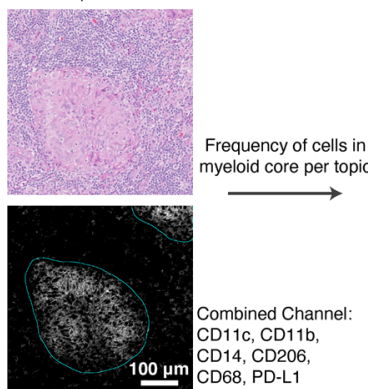

100 um $\mathrm{m}$ CD14, CD206,

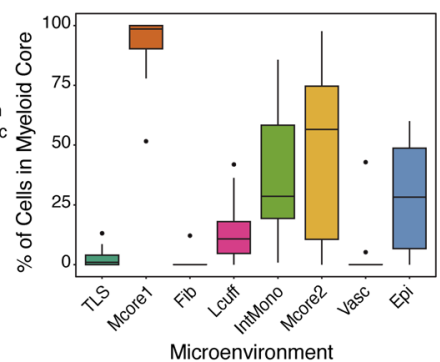

d Microenvironment abundance across FOVs broken down by cell type
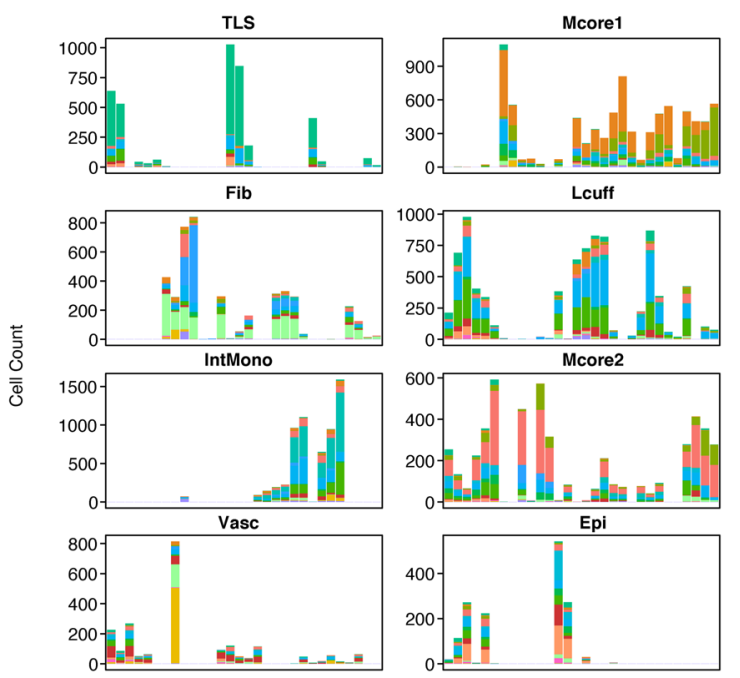

Sample (Patient-ROI, 1->15) b Microenvironment Classification Across Regions Pulmonary Resection: Patients 1-3 Pulmonary Post-mortem: Patients 4-6 Pulmonary Biopsy: Patients 7,8 Extrapulmonary Biopsy: Patients 9-15

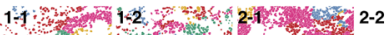

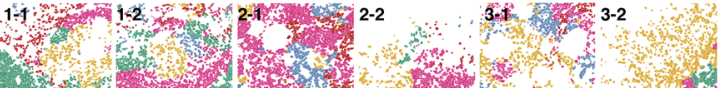

W.

47.

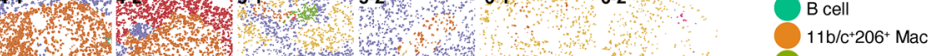

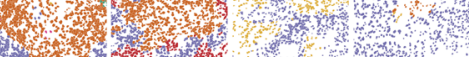
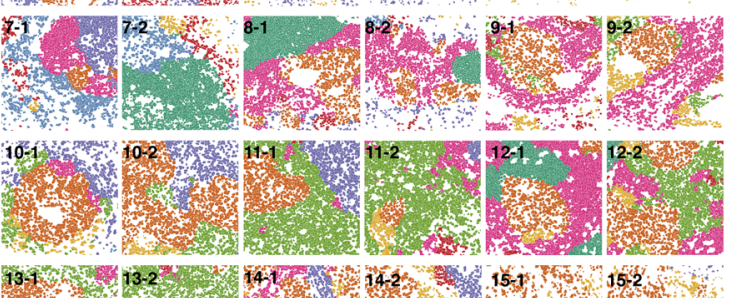

131 , $13-2$

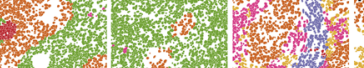

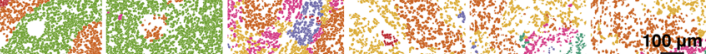

e Microenvironment distributions across specimen types

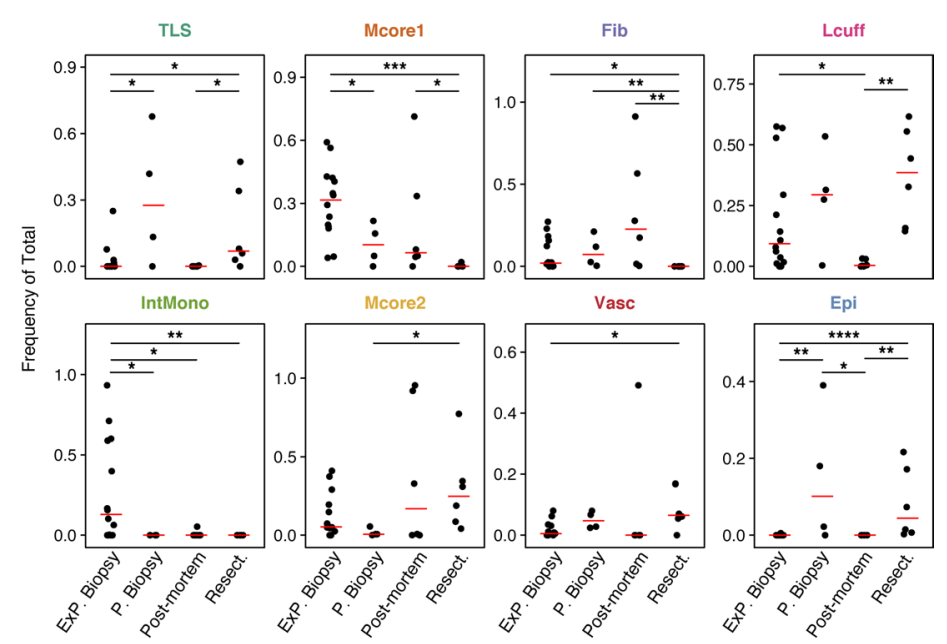

$f \quad$ ME Frequency FOV Clustering

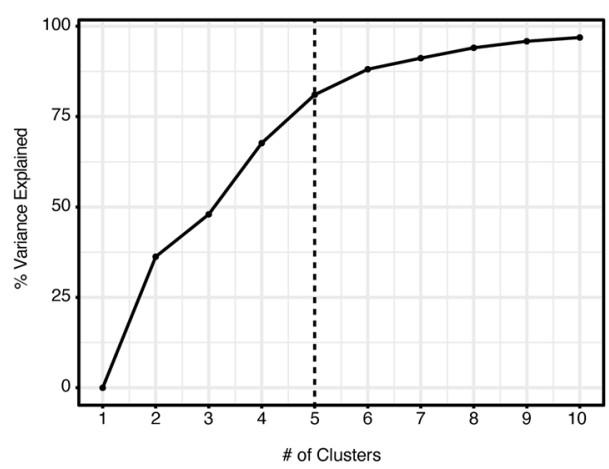

Extended Data Fig. 3 | Spatial protein enrichment and microenvironment modeling of TB granulomas. a, Spatial enrichments of protein expression averaged across all TB granuloma FOVs and visualized as a heatmap hierarchically clustered (Euclidean distance, average linkage). Dashed boxes correspond to modules of protein enrichment corresponding to the myeloid core (green), lymphocytic cuff (blue), and a nonimmune/other niche (pink). b, Max probability maps (MaxPM) for all FOVs. c, Representative hematoxylin \& eosin and combined myeloid channel of a pleural TB FOV for identification of the myeloid core (left) and frequency of cells in the myeloid core across microenvironments (right). Boxplots display the median and interquartile range (IQR, 25-75\%) with whiskers representing the upper- and lower-quartile $\pm 1.5^{\star} I Q R$ ( $n=15$ ). d, Counts of cells broken down by phenotype across all FOVs and microenvironments. e, Frequency of cells across microenvironments broken down by specimen type. Line represents the median $(n=30)$. f. Percent variance explained per clusters based on clustering in Fig. $2 f$. P-values were determined with a Wilcoxon Rank Sum Test (two-tailed) where: $n s p>0.05,{ }^{\star} p<0.05,{ }^{\star \star} p<0.01,{ }^{\star \star \star} p<0.001,{ }^{\star \star \star \star} p<0.0001$. 
a Percent IDO1 and PD-L1+ Cells by Region and Subset
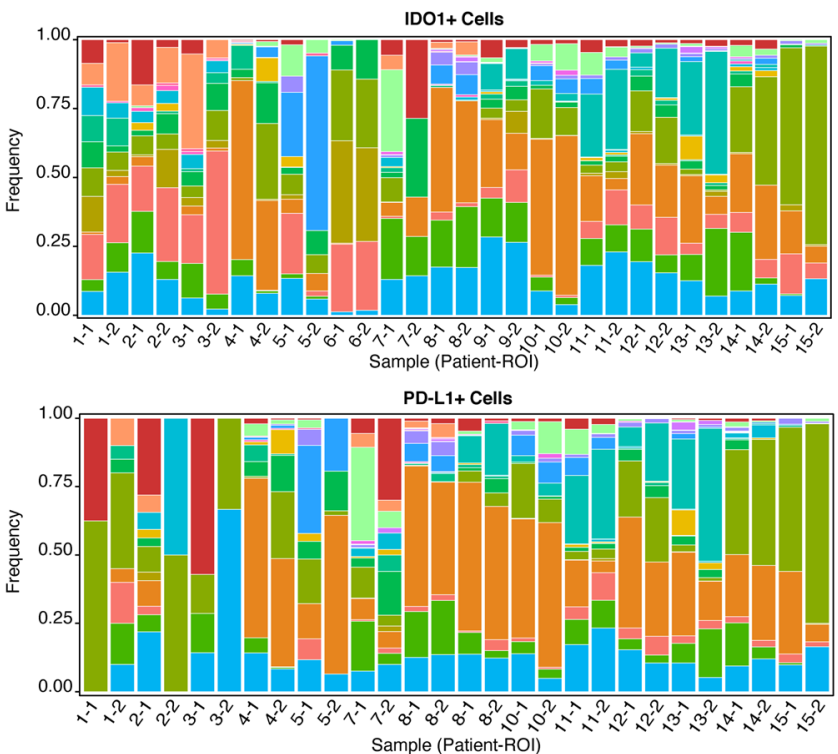

c Percent IDO1 and PD-L1+ Myeloid Cells by Cohort
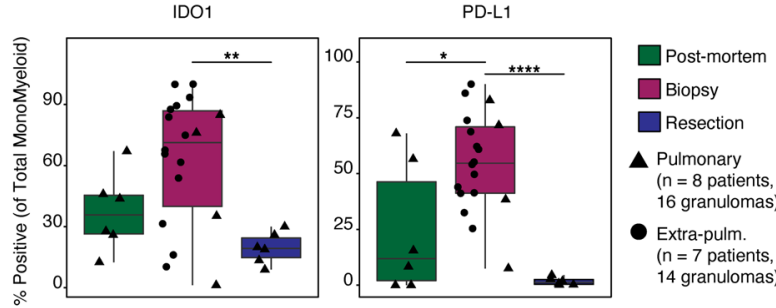

b Expression of IDO1 and PD-L1 across subsets

CD4 T cell CD8 T cell B cell

$11 \mathrm{~b} / \mathrm{c}^{+206+} \mathrm{Mac}$ CD11 $\mathrm{C}^{+} \mathrm{DC} / \mathrm{M}$ ono $\mathrm{CD}_{14} 4^{+} \mathrm{CD} 16^{+} \mathrm{Mono}$ Other Immune Endothelial $\mathrm{CD} 68^{+} \mathrm{Mac}$
$\mathrm{CD} 206^{+} \mathrm{Mac}$ Fibroblast Epithelial Neutrophil $\mathrm{CD} 209^{+} \mathrm{DC}$ $\mathrm{CD} 163^{+} \mathrm{MaC}$ Mast Cell Treg Yठ T cell Giant Cell
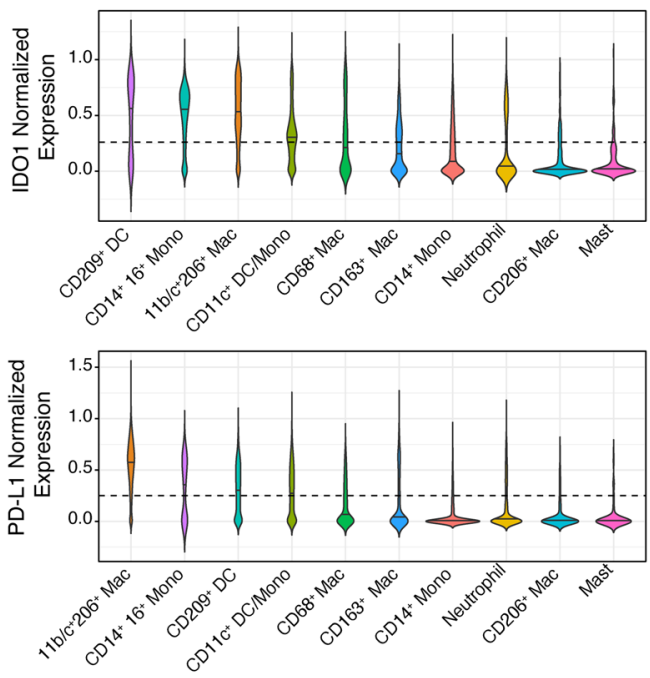

\begin{tabular}{|c|c|c|}
\hline Cohort & Pearson $\mathbf{r}$ & $\mathrm{p}$-value \\
\hline Pulmonary (all) & 0.53 & $\mathrm{p}<2.2 \mathrm{e}-6$ \\
Extra-Pulmonary & 0.62 & $\mathrm{p}<2.2 \mathrm{e}-6$ \\
Resection & 0.22 & $\mathrm{p}<2.2 \mathrm{e}-6$ \\
Biopsy & 0.66 & $\mathrm{p}<2.2 \mathrm{e}-6$ \\
Post-mortem & 0.56 & $\mathrm{p}<2.2 \mathrm{e}-6$ \\
\hline
\end{tabular}

e IDO1 and PD-L1 in Neutrophils and Epithelium

Number of Giant Cells across regions

g

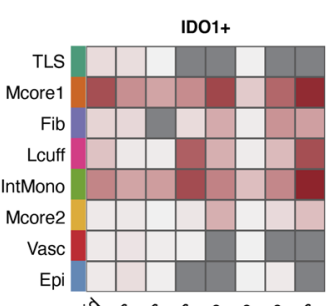

PD-L1

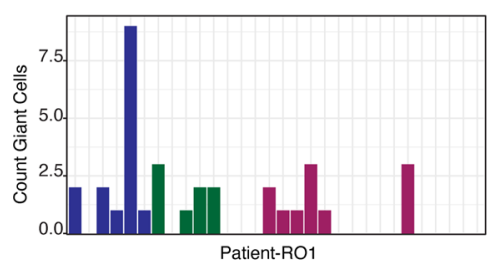

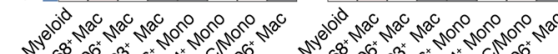

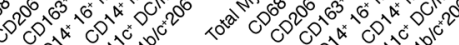

Fequency of Positive Cells

$0,0,1,10$
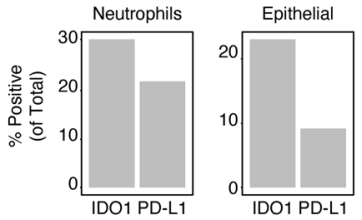

Epithelial

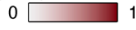

$<1 \%$ of cell subset
Relationship between Tregs, IDO1, and PD-L1 in Mcore1
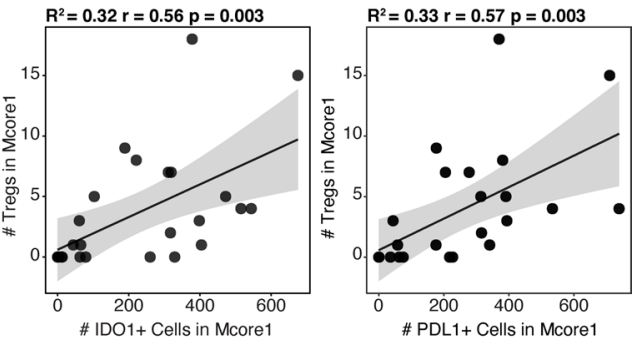

i PD1+ Lymphocytes Across Topics j Count of IFN $\mathrm{Y}+$ cell across regions
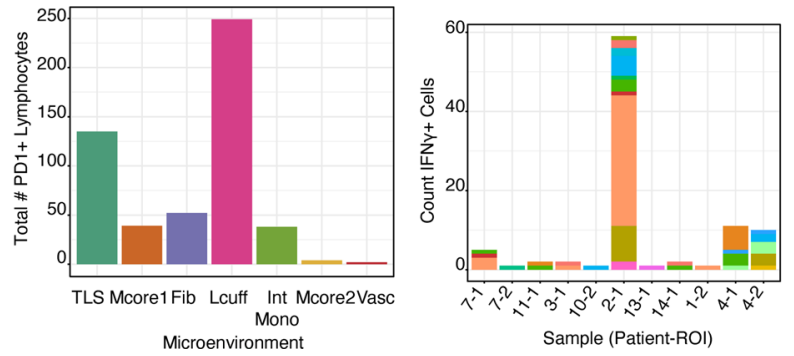

Extended Data Fig. 4 | See next page for caption. 
Extended Data Fig. 4 | Immunoregulatory protein expression in TB granulomas. a, Frequency of cells positive for IDO1 (top) or PD-L1 (bottom) broken down by FOV and cell phenotype. b, Normalized expression of IDO1 (top) and PD-L1 (bottom) for major myeloid subsets ordered by decreasing median expression value. Dashed line indicates the cutoff for positivity for IDO1 (cutoff $=0.26$ ) and PD-L1 (cutoff $=0.25$ ). $\mathbf{c}$, Frequency of IDO1+ or PD-L $1^{+}$cells (of total cells) across specimen type (postmortem specimen = green, biopsy = red, therapeutic resection = blue) with dot shape representing organ site (lung = triangle, extrapulmonary = circle). $\mathbf{d}$, Pearson correlation coefficient and p-value determined by t-test (two-tailed) broken down by specimen type. e, Frequency of neutrophils (left) and epithelial cells (right) positive for PD-L1 or IDO1 across all FOVs. f, The count of giant cells across all regions, colored by specimen origin as in $\mathrm{c}$. $\mathbf{g}$, The frequency of IDO1+ and PD-L1+ myeloid cells for all non-granulocytic myeloid cell subsets across ME. Any ME with fewer than $1 \%$ of the total cell subset is shaded gray. $\mathbf{h}$, Linear relationship between count of Tregs in Mcore1 with count of IDO1+(left) and PD-L1+ (right) cells in Mcore1. Linear regression (black solid line) with 95\% confidence interval (grey silhouette) displayed. Significance was established with a two-tailed t-test. $\mathbf{i}$, Count of PD-1+ cells across all MEs with $>0$ positive cells. $\mathbf{j}$ The count of IFN $\gamma^{+}$cells in all FOVs with $>0$ positive cells, colored by cell type. Unless otherwise specified all $p$ values were determined with a Wilcoxon Rank Sum Test (two-tailed) where: ${ }^{\star} p<0.05,{ }^{\star \star} p<0.01,{ }^{\star \star \star \star} p<0.0001$. 
a In situ Cytokine Analysis

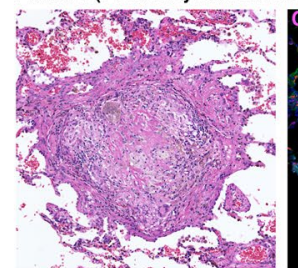

$M E_{\text {Mcorei }} M E_{F i b} M E_{\text {Lcuff }} M E_{T L S} M E$

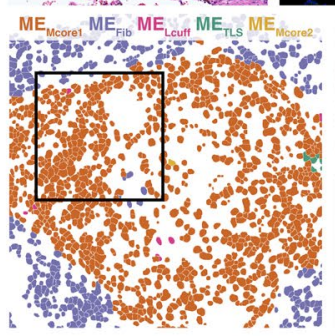

e
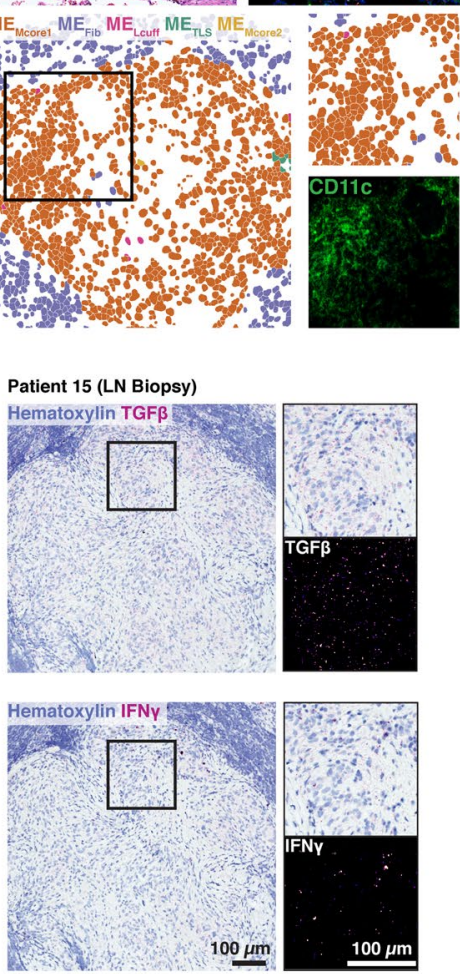
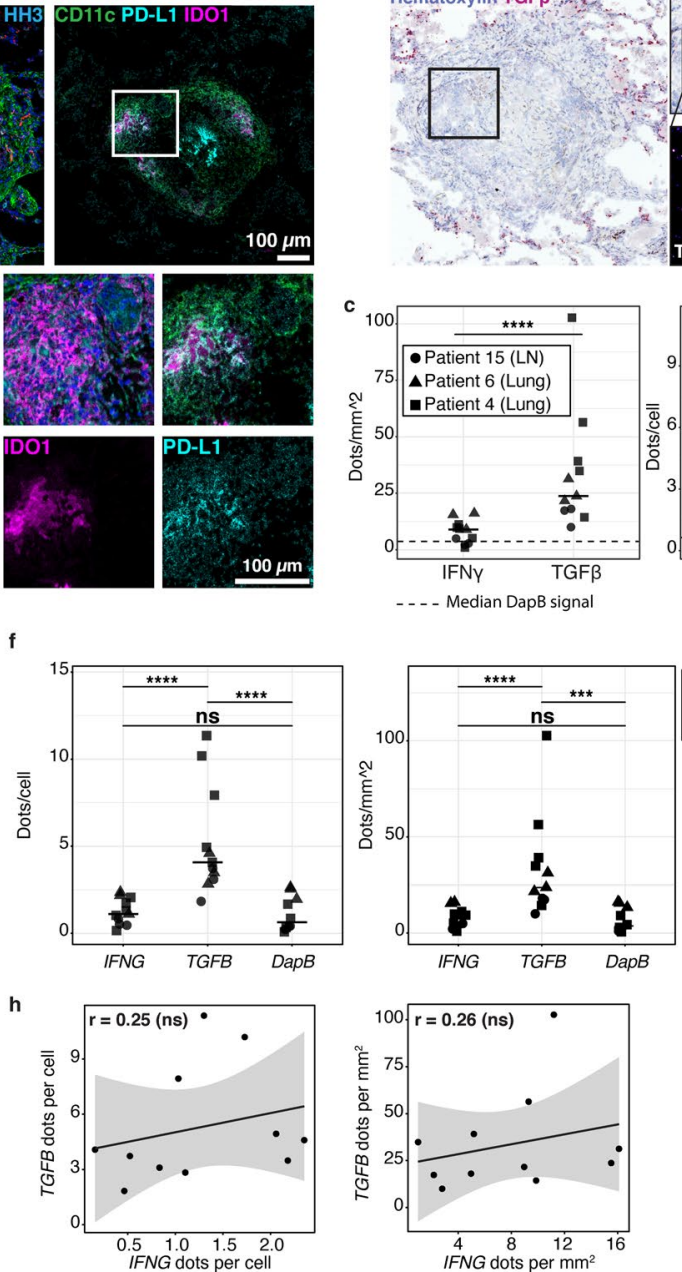

- . - - Median DapB signal

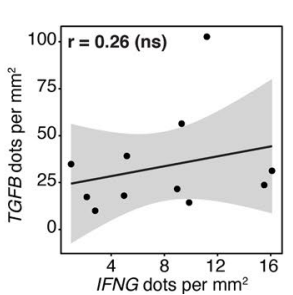

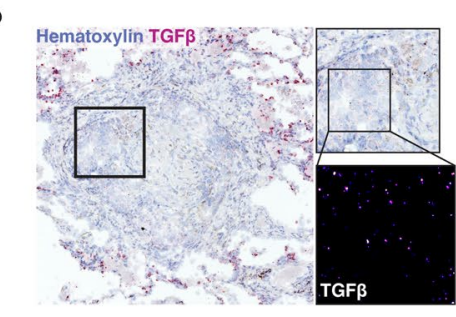
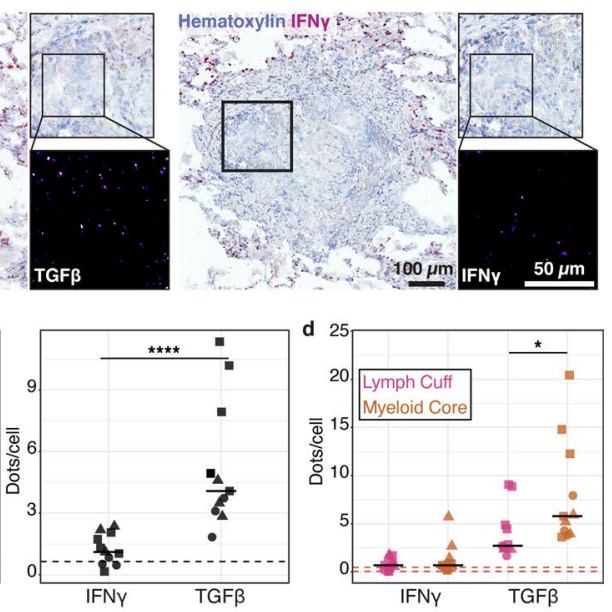

g

\section{)}

Extended Data Fig. 5 | In situ hybridization of TGFB and IFNG transcripts in human TB granulomas. a, Representative images from a pulmonary TB granuloma section showing hematoxylin \& eosin staining (upper left), MIBI-TOF images (left: CD45=magenta, VIM =cyan, CD31=red, $\alpha S M A=$ green, $\mathrm{HH} 3=$ blue, right: $\mathrm{CD} 11 \mathrm{c}=$ green, $\mathrm{PD}-\mathrm{L} 1=$ cyan, IDO1 = magenta) and ME assignment with zoomed insets indicated by white or black boxes.

b, Representative chromogenic ISH of TB granuloma from a with zoomed inset indicated by black box and colored with fire LUT. c, Quantification of transcripts per area (left) and dots per cell (right). Solid line represents the median for probe $(n=11)$. Dashed line represents the median DapB signal. d, Quantification of transcripts per cell broken down by granuloma region (orange $=$ myeloid core, pink $=$ lymphocytic cuff). Solid line represents the median for probe $(n=11)$. Dashed line represents the median DapB signal per region type. e, Representative image of chromogenic ISH in a TB granuloma with zoomed insets indicated by black box. ISH signal colored with fire LUT. f, Quantification of TGFB, IFNG, and DapB transcripts per cell (left) and per area (right). Solid line represents the median for probe $(n=11)$. $\mathbf{g}$, Proportion IFN $\gamma^{+}$cells measured by MIBI-TOF in ME $\mathrm{Mcore}$ as a fraction of total IFN $\gamma^{+}$ cells per ROI. h, Linear relationship between IFNG and TGFB dots per cell (left) and dots per $\mathrm{mm}^{2}$ (right). Linear regression (black solid line) with 95\% confidence interval (grey silhouette) displayed. Pearson correlation coefficient displayed. Significance was established with a t-test (two-tailed). Unless specified, all $p$ values were determined with a Wilcoxon Rank Sum Test (two-tailed) where: ns $p>0.05,{ }^{\star} p<0.05,{ }^{\star \star \star} p<0.001,{ }^{\star \star \star \star \star} p<0.0001$. 
a
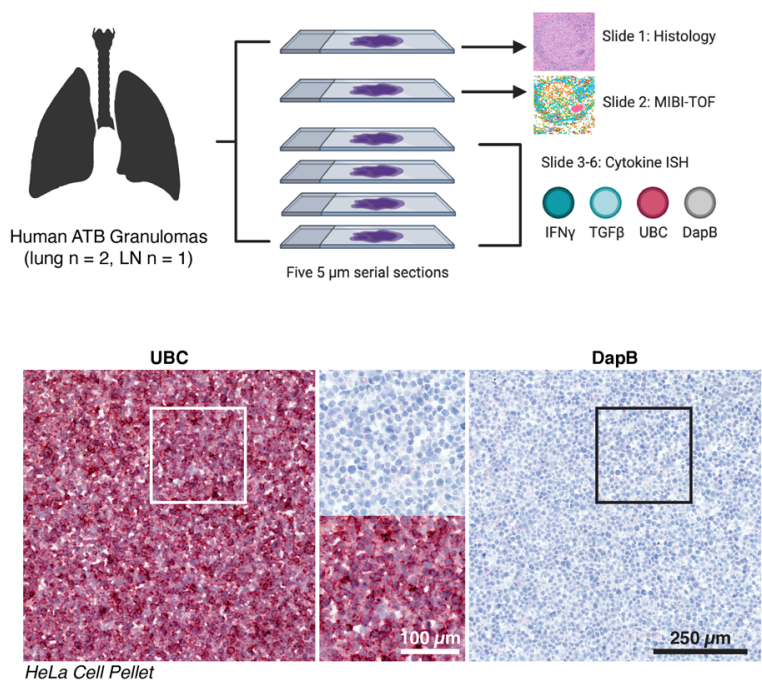

$c$
DapB

UBC

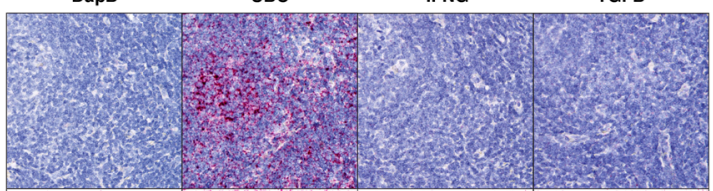

Spleen (40x)
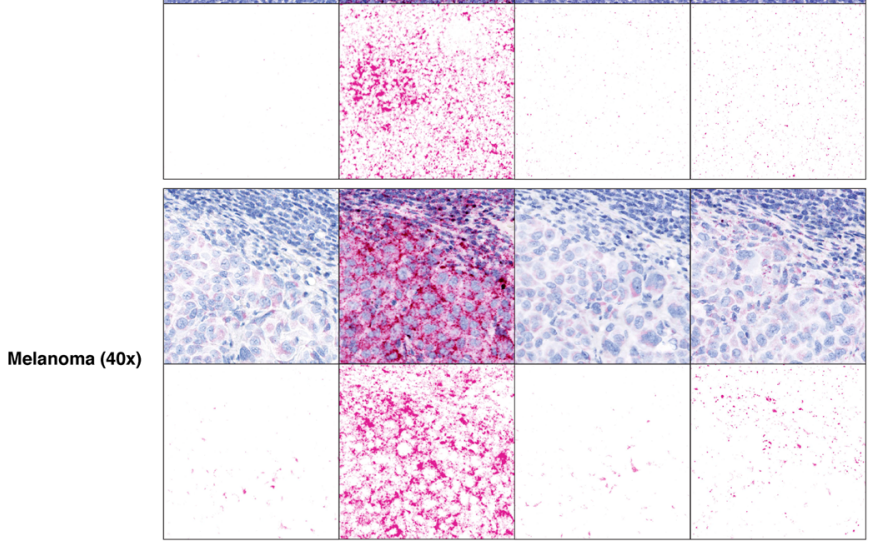

d

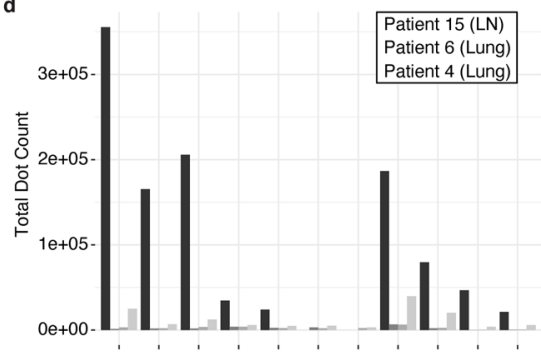

15-1 15-2 15-3 6-1 6-2 6-3 4'-1 4-2 4 4-3 4 4-4 4 4-5

Patient-ROI

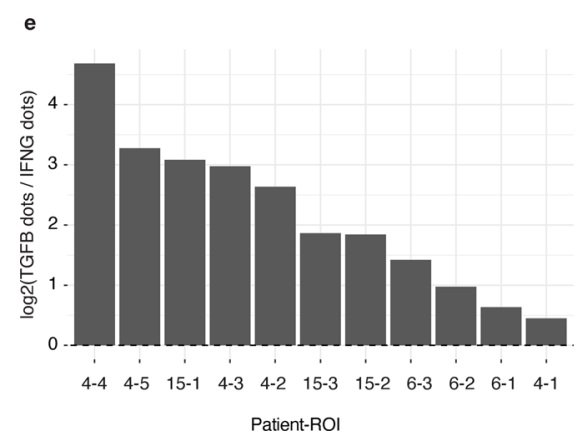

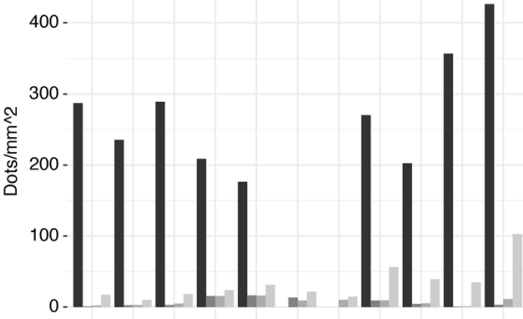

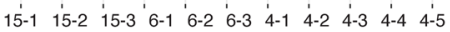

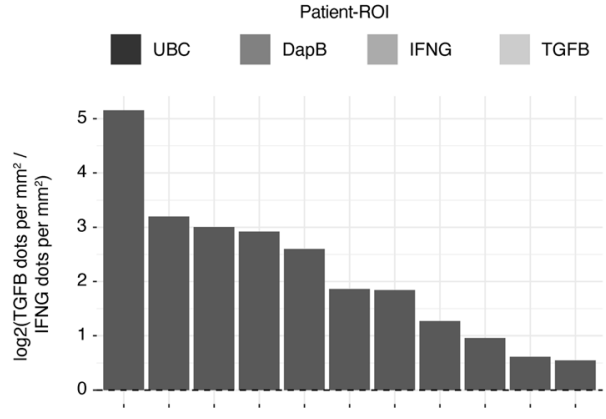

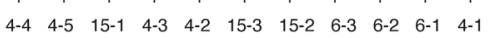

Patient-ROI

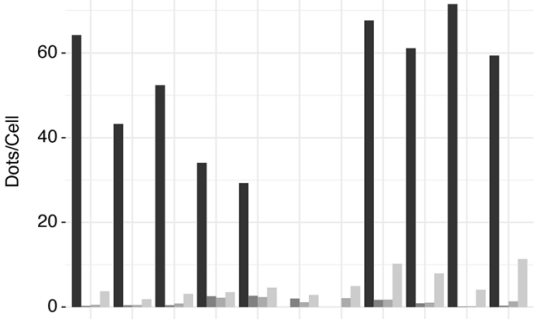

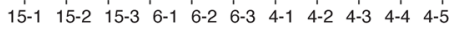

Patient-ROI

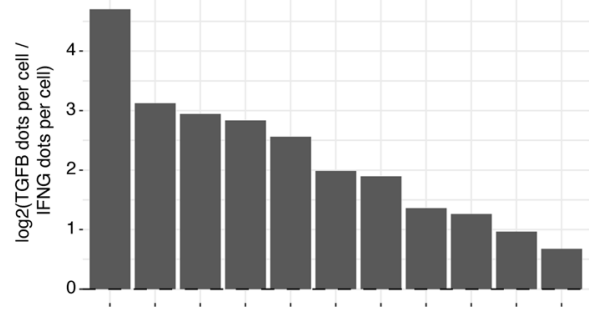

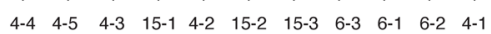

Patient-ROI

Extended Data Fig. 6 | In situ hybridization workflow and supplemental analysis. a, Conceptual overview of combined ISH and MIBI-TOF experimental workflow. b, Representative images of control probes UBC (+) and DapB (-) in HeLa cell pellets. c, Representative images of all probes in human spleen and melanoma at 40x magnification. d, Grouped bar plots of total counts (left), area-normalized counts (middle), and cell-normalized counts (right) for all granuloma regions analyzed. e, The ratio of TGFB: IFNG transcripts as a log2 fold-change for total counts (left), area-normalized counts (middle), and cell-normalized counts (right) for all granuloma regions analyzed. 
a H\&E Serial Sections of Sarcoidosis Cohort

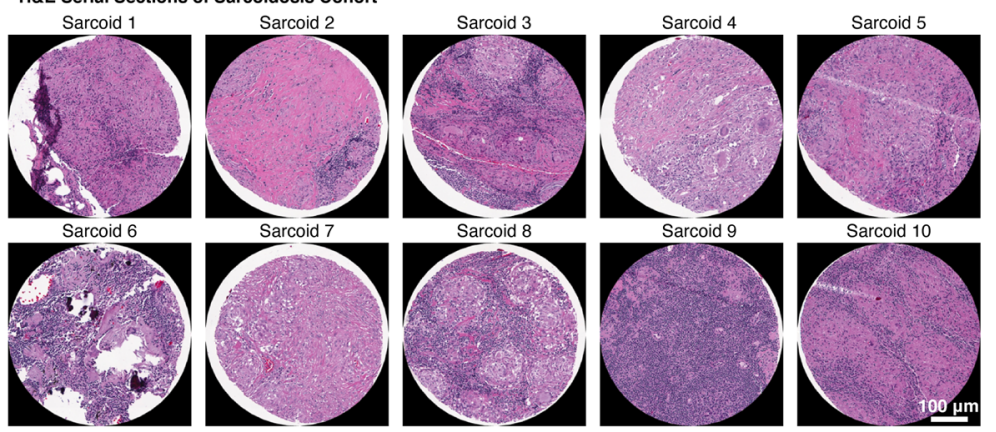

c Cell composition in TB v Sarcoidosis-All Subsets

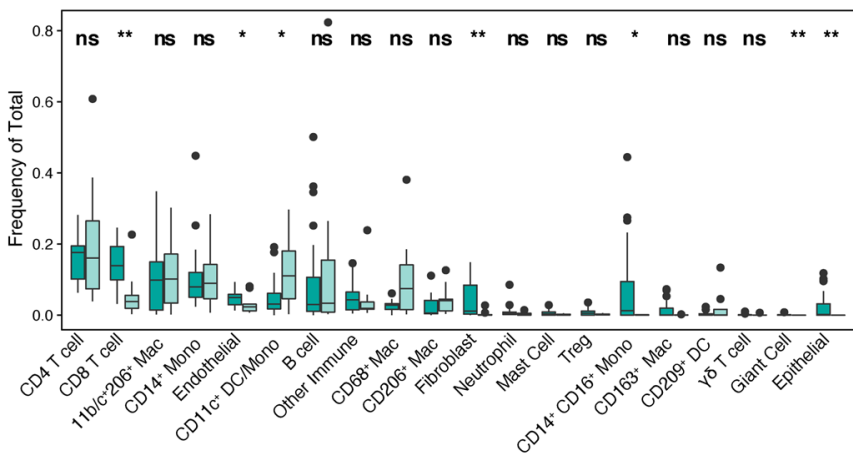

e IDO1 and PDL1 Chromogenic IHC Across Granuloma Types

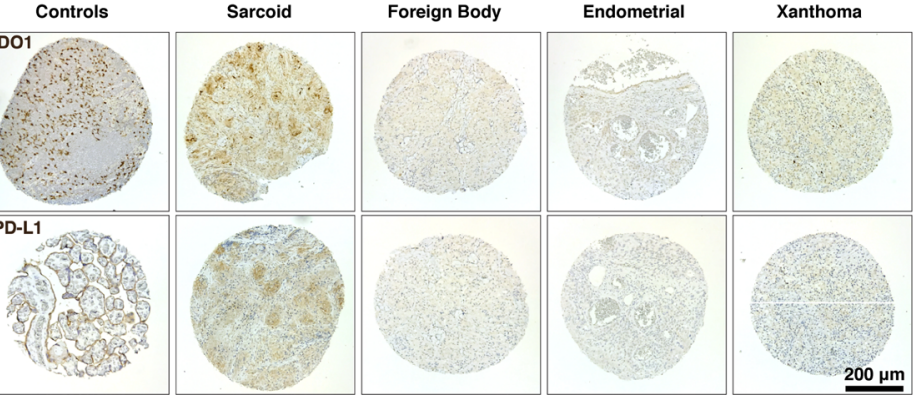

b Distrubution of immune cells across regions

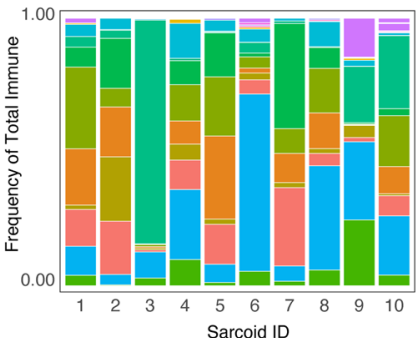

d Composition of PD-L1+ Cells in Sarcoidosis

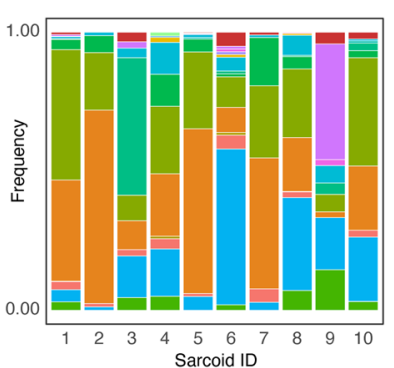

CD4 T cell

CD8 T cell

CD14+ Mono

B cell

$11 \mathrm{~b} / \mathrm{c}^{+} 206^{+} \mathrm{Mac}$

CD11c + DC/Mono

$\mathrm{CD} 14^{+} \mathrm{CD} 16^{+}$Mono

Other Immune

$\mathrm{CD} 68+\mathrm{Mac}$

$\mathrm{CD}^{206+\mathrm{MaC}}$

Neutrophil

CD209+DC

$\mathrm{CD}_{163}+\mathrm{MaC}$

Mast Cell

Treg

Y $\delta$ T cell

Giant Cell

f IDO1 and PDL1 Expression in Pulmonary Mycobacterium Avium

Pulmonary $M$. avium H\&E

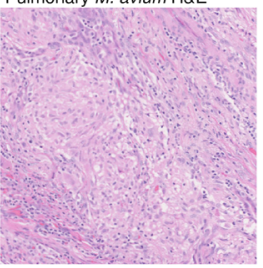

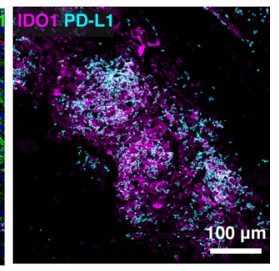

Extended Data Fig. 7 | Immunoregulatory protein expression in nontuberculous granulomas. a, Hematoxylin \& eosin-stained sections of sarcoidosis granuloma FOVs. b. Frequency of immune cell subsets out of total immune cells broken down by sarcoidosis FOV. c, Comparison of cell type frequency (out of total cells) between tuberculosis (dark green) and sarcoidosis (light green). Boxplots display the median and interquartile range (IQR, 25-75\%) with whiskers representing the upper- and lower-quartile $\pm 1.5^{\star} \mathrm{IQR}(T B n=30$, sarcoid $n=10)$. P-values were determined with a Wilcoxon Rank Sum Test (two-tailed) where: $n s p>0.05,{ }^{\star} p<0.05$, and ${ }^{\star \star} p<0.01$. d, Frequency of PD-L1+ cells across all sarcoidosis FOVs broken down by cell subset. e, Representative immunohistochemistry images of PD-L1 or IDO1 (brown) of controls (top = spleen, bottom=placenta), a sarcoid granuloma, xanthoma granuloma, foreign body lesion, and endometrial lesion with hematoxylin nuclear counterstaining (purple). f, Hematoxylin and eosin (left) and MIBI-TOF staining for major cell lineage markers (middle) or IDO1 (magenta) and PD-L1 (cyan) (right) of a representative pulmonary Mycobacterium avium FOV. 
a Effect Size All Genes Across Infection Stage

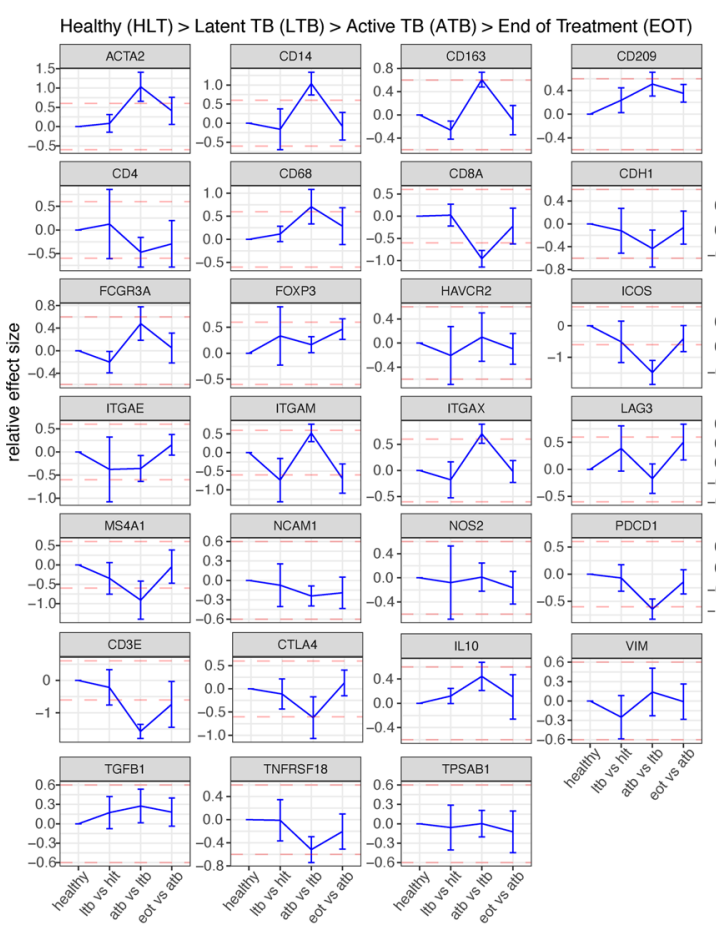

d

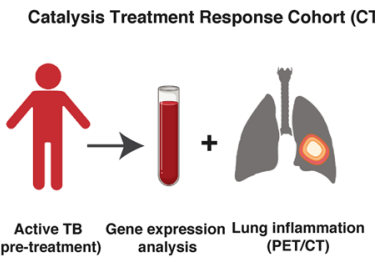

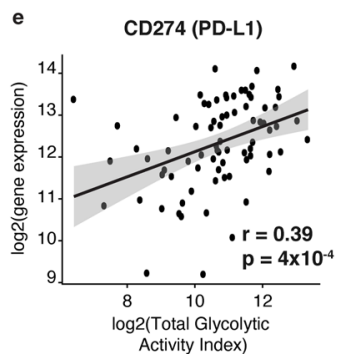

b CD274 Expression in individual progressors and non-progressors

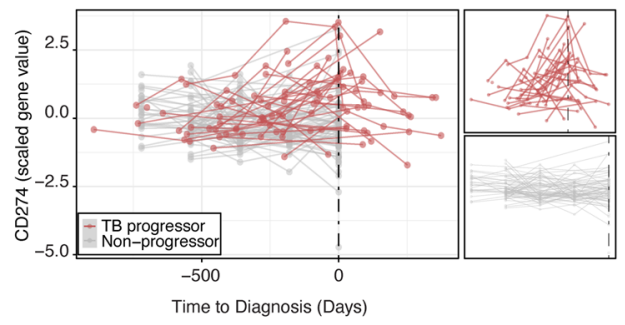

c
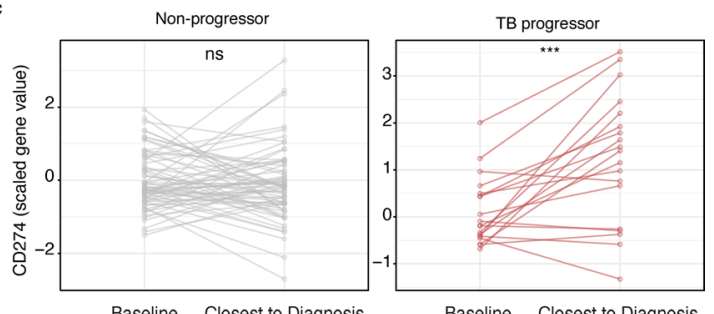

f CTRC PD-L1 and PD-L2 Expression

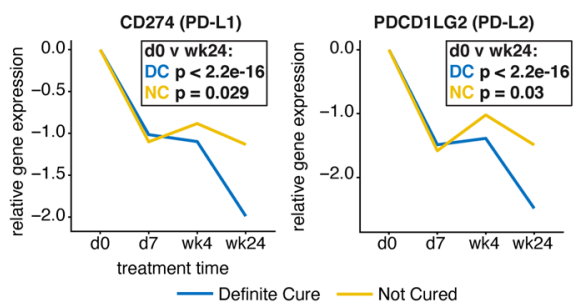

Extended Data Fig. 8 | Transcriptomic analysis of peripheral blood in TB patients. a, Gene effect sizes in latent TB ( $n=173$ ) versus healthy controls $(n=197)$, latent TB $(n=372)$ versus active TB $(n=479)$, and active TB $(n=168)$ versus end-of-treatment $(n=160)$. Bars represent the mean and standard deviation. Dashed red lines represent a relative effect size of $0.6 . \mathbf{b}$, CD274 scaled gene expression in progressors (red) and non-progressors (gray) with zoomed insets displaying groups individually. c, CD274 expression in progressors at the earliest recorded time point prior to progression and the closest time point to diagnosis with ATB (within 30 days). P-values were calculated with a one-sided paired sample t-test. d, Conceptual overview of the Catalysis Treatment Response Cohort (CTRC). e, Correlation between PD-L1 gene expression and total glycolytic activity index (TGAI) represented as log2-transformed values. Linear regression (black line) with $95 \%$ confidence interval (grey) displayed. A Pearson correlation of $0.39\left(p=4 \times 10^{-4}\right)$ is displayed below the linear fit. Significance was established with a t-test (two-tailed). f PD-L1 (left) and PD-L2 (right) gene expression across treatment time broken down by cure status (blue $=$ definite cure and yellow $=$ no cure). Line represents mean expression in each time point, connected across time points. P-value determined with Student's t-test for PD-L1 expression at d0 versus wk24 in the definite cure $(D C, n=71)$ and not-cured (NC, $n=7)$ groups. 
Extended Data Table 1 | Tuberculosis granuloma cohort clinical information

\begin{tabular}{|c|c|c|c|c|c|c|c|c|c|c|}
\hline Patient ID & Source & Country & Specimen & Tissue & Sex & Age range (yrs) & TB Type & HIV Status & Treatment & Macro/Microscopic Features \\
\hline & Albert Luthuli Central Hospital & South Africa & Resection & Lung & M & $18-64$ & TB & Negative & NA & $\begin{array}{l}\text { Left lung pneumonectomy, no } \\
\text { visible tubercles, extensive fibrosis } \\
\text { with bronchiectasis and } \\
\text { associated hemorrhage }\end{array}$ \\
\hline & Albert Luthuli Central Hospital & South Africa & Resection & Lung & M & 265 & MDR-TB & Not Reported & NA & $\begin{array}{l}\text { Left upper lobectomy, lung } \\
\text { contains large areas of necrotizing } \\
\text { granulomatous inflammation, acid- } \\
\text { fast bacilli are present }\end{array}$ \\
\hline & Albert Luthuli Central Hospital & South Africa & Resection & Lung & $\mathrm{M}_{\mathrm{M}}$ & $18-64$ & TB & Negative & NA & \begin{tabular}{|l|} 
Left lung pneumonectomy, \\
bronchiectatic with multiple \\
irregularly shaped tuberles, \\
granulomas are composed of \\
central caseative type necrosis, \\
paucibacillary
\end{tabular} \\
\hline \multicolumn{11}{|c|}{ Specimens } \\
\hline Patient ID & Source & Country & Specimen & Tissue & Sex & Age range (yrs) & AFB & Culture & IHC for Mtb Antigen & HIV Status \\
\hline 4 & University of Texas Health Science Center & United States & Post-mortem & Lung & $M$ & $18-64$ & Negative & Positive & Positive & Negative \\
\hline 5 & University of Texas Health Science Center & United States & Post-mortem & Lung & $M$ & $18-64$ & Negative & Positive & Positive & Negative \\
\hline & University of Texas Health Science Center & United States & Post-mortem & Lung & $\mathrm{M}$ & $18-64$ & Positive & Positive & Positive & Negative \\
\hline
\end{tabular}

\begin{tabular}{|c|c|c|c|c|c|c|c|c|c|}
\hline Patient ID & Source & Country & Specimen & Tissue & Sex & Age range (yrs) & AFB & PCR & HIV Status \\
\hline & Stanford Hosptial & United States & Biopsy & & & & Positive & Positive & Negative \\
\hline & Stanford Hosptial & United States & Biopsy & Lung & M & 265 & Positive & Positive & Not Reported \\
\hline & Stanford Hosptial & United States & Biopsy & Vertebral Body 77 & M & not reported & Positive & Positive & Negative \\
\hline 10 & Stanford Hosptial & United States & Biopsy & Pleura & $\mathrm{M}$ & 265 & Positive & Positive & Negative \\
\hline 11 & Stanford Hosptial & United States & Biopsy & Pleura & $\mathrm{M}$ & $18-64$ & Positive & Positive & Negative \\
\hline 12 & Stanford Hosptial & United States & Biopsy & Pleura & $\mathrm{M}$ & 18-64 & Positive & Positive & Negative \\
\hline 13 & Stanford Hosptial & United States & Biopsy & Endometrium & $F$ & 265 & Positive & Positive & Negative \\
\hline 14 & Stanford Hosptial & United States & Biopsy & Lymph Node & $\mathrm{M}$ & $18-64$ & Positive & Positive & Negative \\
\hline 15 & Stanford Hosptial & United States & Biopsy & Lymph Node & M & $118-64$ & Positive & Positive & Negative \\
\hline
\end{tabular}


Extended Data Table 2 | Multiplexed imaging antibody panel staining conditions and low-level processing parameters

\begin{tabular}{|c|c|c|c|c|c|c|c|c|c|c|c|c|}
\hline \multicolumn{7}{|c|}{ Panel 1 (overnight stain) } & \multicolumn{6}{|c|}{ Parameters for analysis } \\
\hline Antibody target & Provider & Catalog Number & Lot & Clone & \begin{tabular}{|l} 
Mass \\
channel
\end{tabular} & $\begin{array}{l}\text { Titer } \\
(\mu \mathrm{g} / \mathrm{mL})\end{array}$ & Start & Stop & NoiseT 1 & NoiseT 2 & NoiseT 3 & AggFilter \\
\hline Collagen-1 & Abcam & $a b 215969$ & GR296572-1 & EPR7785 & $141 \mathrm{Pr}$ & 0.50 & 140.7 & 141.2 & 5 & 5 & 4 & 150 \\
\hline Lag3 & LSBio & LS-C18692 & 113549 & 17B4 & $142 \mathrm{Nd}$ & 0.50 & 141.7 & 142.2 & 5 & 5 & 5 & 200 \\
\hline CD4 & Abcam & ab181724 & GR32155375-1 & EPR6855 & $143 \mathrm{Nd}$ & 0.50 & 142.7 & 143.2 & 6 & 6 & 5 & 100 \\
\hline CD14 & Cell Signaling & $56082 \mathrm{BF}$ & 2 & D7A2T & $144 \mathrm{Nd}$ & 0.50 & 143.7 & 144.2 & 4 & 4 & 4 & 100 \\
\hline Foxp3 & BD Biosciences & 624084 & 8099783 & 236A/E7 & $146 \mathrm{Nd}$ & 1.00 & 145.7 & 146.2 & 5 & 6 & 5 & 200 \\
\hline PD1 & Cell Signaling & $86163 \mathrm{BF}$ & 2 & D4W2J & $147 \mathrm{Sm}$ & 1.00 & 146.7 & 147.2 & 6 & 6 & 4 & 100 \\
\hline CD31 & Abcam & ab207091 & GR241753-2 & EP3095 & $148 \mathrm{Nd}$ & 0.50 & 147.7 & 148.2 & 3.5 & 3.5 & 3.5 & 100 \\
\hline PD-L1-biotin & Cell Signaling & 13684BF & 2 & E1L3N & NA & 1.00 & NA & NA & NA & NA & NA & NA \\
\hline E-Cadherin & Abcam & ab213606 & not recorded & EP700Y & $150 \mathrm{Nd}$ & 0.25 & 149.7 & 150.2 & 5 & 5 & 3.5 & 180 \\
\hline $\begin{array}{ll}\text { Ki67 } \\
\end{array}$ & Cell Signaling & 9449BF & 2 & $8 \mathrm{D} 5$ & $151 \mathrm{Eu}$ & 0.25 & 150.7 & 151.2 & 4 & 4 & 4 & 100 \\
\hline CD209/DC-SIGN & BD Biosciences & 624084 & not recorded & DCN46 & $152 \mathrm{Sm}$ & 0.13 & 151.7 & 152.2 & 4.5 & 4.5 & 3.5 & 150 \\
\hline CD206 & Cell Signaling & 91992BF & 2 & E2L9N & $153 \mathrm{Eu}$ & 0.50 & 152.7 & 153.2 & 5 & 5 & 4.5 & 140 \\
\hline TCRס & Santa Cruz & sc- $100289 X$ & $\mathrm{H} 2317$ & $\mathrm{H}-41$ & $154 \mathrm{Sm}$ & 1.00 & 153.7 & 154.2 & 4 & 4 & 3.5 & 200 \\
\hline iNOS & Spring Bioscience & M4264 & 170802 & SP126 & $155 \mathrm{Gd}$ & 0.50 & 154.7 & 155.2 & 5 & 5 & 3 & 200 \\
\hline \begin{tabular}{|l|} 
CD68 \\
\end{tabular} & Abcam & 76437BF & 2 & D4B9C & $156 \mathrm{Gd}$ & 0.13 & 155.7 & 156.2 & 5 & 5 & 4.5 & 120 \\
\hline CD36 & Abcam & 14347BF & 2 & D8L9T & $157 \mathrm{Gd}$ & 0.50 & 156.7 & 157.2 & 4.5 & 4.5 & 4 & 100 \\
\hline CD8 & Cell Marque & 108M-OEM1404 & 1514101 & C8/144B & $158 \mathrm{Gd}$ & 0.25 & 157.7 & 158.2 & 5 & 5 & 5 & 75 \\
\hline CD3 $\varepsilon$ & Cell Signaling & $85061 \mathrm{BF}$ & 4 & D7A6E & $159 \mathrm{~Tb}$ & 0.25 & 158.7 & 159.2 & 6 & 6 & 5 & 100 \\
\hline ID01 & Spring Bioscience & M5604.C & 170215 & SP260 & 160Gd & 0.50 & 159.7 & 160.2 & 7 & 7 & 4.5 & 100 \\
\hline CD11c & Abcam & ab216655 & GR3210349-1 & EP1347Y & 161Dy & 0.25 & 160.7 & 161.2 & 5 & 5 & 5 & 100 \\
\hline CD163 & Cell Signaling & 93498BF & 2 & D5U1J & 163Dy & 2.00 & 162.7 & 163.2 & 4 & 4 & 5 & 100 \\
\hline CD20 & Cell Marque & 120M-OEM1404 & 1429304 & L26 & $164 \mathrm{Er}$ & 0.50 & 163.7 & 164.2 & 4 & 4 & 5 & 100 \\
\hline CD16 & Cell Signaling & $24326 \mathrm{BF}$ & 2 & D1N9L & $165 \mathrm{Ho}$ & 1.00 & 164.7 & 165.2 & 5 & 5 & 4 & 100 \\
\hline IFNy & Abcam & ab218890 & GR3191590-2 & IFNG/466 & $166 \mathrm{Er}$ & 1.00 & 165.7 & 166.2 & 5 & 5 & 4 & 200 \\
\hline HLA-DR-DQ-DP & Abcam & ab7856 & GR3191247-1 & CR3/43 & $167 \mathrm{Er}$ & 0.25 & 166.7 & 167.2 & 5 & 5 & 4.5 & 100 \\
\hline CD11b & Abcam & $a b 187537$ & GR286344-1 & EP1345Y & $168 \mathrm{Er}$ & 0.25 & 167.7 & 168.2 & 5 & 5 & 4.5 & 100 \\
\hline CD45 & Cell Signaling & 13917BF & 2 & D9M8I & $169 \mathrm{Tm}$ & 0.50 & 168.7 & 169.2 & 5 & 5 & 5 & 200 \\
\hline H3K9Ac & Cell Signaling & 9649BF & 12 & C5B11 & $170 \mathrm{Er}$ & 1.00 & 169.7 & 170.2 & 3 & 3 & 3 & 200 \\
\hline Keratin (pan) & ThermoFisher & MS-343-PABX & $343 \times 1801 \mathrm{~A}$ & AE1/AE3 & $171 \mathrm{Yb}$ & 1.00 & 170.7 & 171.2 & 4 & 4 & 4 & 150 \\
\hline CD103 & Abcam & $a b 221210$ & GR3175670-1 & EPR4166(2) & $172 \mathrm{Yb}$ & 0.50 & 171.7 & 172.2 & 5 & 5 & 4.5 & 200 \\
\hline MPO & R\&D Systems & AF3667 & YBZ0217091 & polyclonal & $174 \mathrm{Yb}$ & 0.75 & 173.7 & 174.2 & 3 & 3 & 3 & 180 \\
\hline $\mathrm{N}+/ \mathrm{K}+\mathrm{ATPase}$ & Abcam & ab167390 & GR3229163-1 & EP1845Y & $175 \mathrm{Lu}$ & 1.00 & 174.7 & 175.2 & 5 & 5 & 5 & 150 \\
\hline HLA Class I & Abcam & ab70328 & GR307795-2 & EMR8-5 & $176 \mathrm{Yb}$ & 1.00 & 175.7 & 176.2 & 4 & 4 & 4 & 100 \\
\hline
\end{tabular}

\begin{tabular}{|c|c|c|c|c|c|c|c|c|c|c|c|c|}
\hline Antibody target & Provider & Catalog Number & Lot & Clone & \begin{tabular}{|l|} 
Mass \\
channel
\end{tabular} & Titer & Start & Stop & NoiseT 1 & NoiseT 2 & NoiseT 3 & AggFilter \\
\hline $\mathrm{HH} 3$ & Cell Signaling & 4499BF & 7 & $\mathrm{D} 1 \mathrm{H} 2$ & $89 Y$ & 2.00 & 88,7 & 89.2 & 2.5 & 2.5 & 2 & 0 \\
\hline Vimentin & Cell Signaling & $5741 \mathrm{BF}$ & 3 & D2H13 & $113 \ln$ & 2.00 & 112.7 & 113.2 & 3 & 3 & 3 & 150 \\
\hline SMA & Spring Bioscience & M4714.C & not recorded & SP171 & $115 \ln$ & 2.00 & 114.7 & 115.2 & 2.5 & 2.5 & 2 & 200 \\
\hline biotin & Biolegend & 409002 & B232547 & 1D4-C5 & $149 \mathrm{Sm}$ & 2.00 & 148.7 & 149.2 & 5 & 5 & 5 & 75 \\
\hline Chymase & Abcam & ab233729 & GR3218665-1 & EPR13136 & $173 \mathrm{Yb}$ & 0.25 & 172.7 & 173.2 & 1.7 & 1.7 & 2 & 200 \\
\hline \begin{tabular}{|l|} 
Tryptase \\
\end{tabular} & Abcam & ab212156 & GR273336-1 & EPR9522 & $173 \mathrm{Yb}$ & 0.25 & 172.7 & 173.2 & 1.7 & 1.7 & 2 & 200 \\
\hline
\end{tabular}


Extended Data Table 3 | Cell phenotyping criteria

\begin{tabular}{|l|l|}
\hline Subset & Criteria \\
\hline Immune cell & CD45+ \\
\hline Fibroblast & aSMA+ \\
\hline Endothelial Cell & CD31+ \\
\hline Epithelial Cell & PanCK+ ECAD+/- \\
\hline B cell & CD20+ \\
\hline CD8 T cell & CD3+ CD8+ \\
\hline CD4 T cell & CD3+ CD4+ Foxp3- \\
\hline Treg & CD3+ CD4+ Foxp3+ \\
\hline gd T cell & CD3+ TCR-d+ \\
\hline Neutrophil & MPO+ CD11b+/- \\
\hline Mast Cell & Chymase/Tryptase+ \\
\hline Monocyte-derived Cell & CD14+ \\
\hline CD163+ Mac & CD163+ CD16+/- CD209+/- \\
\hline CD209+ DC & CD209+ CD68- CD163- CD11C+/- \\
\hline $\mathbf{1 1 b / c + 2 0 6 + ~ M a c ~}$ & CD68+CD206+ CD11c+ CD11b+ CD16+/- \\
\hline CD68+ Mac & CD68+ \\
\hline CD206+ Mac (alveolar like) & CD45dim CD14- CD206+ \\
\hline CD11c+ DC/Mono & CD14+ CD11c+ CD68- \\
\hline CD14+ CD16+ Mono & CD14+ CD16+ \\
\hline Giant Cell & Multinucleated \\
\hline Tfh & CD3+ PD-1+ B cell follicle \\
\hline Immune Other & CD45+ Lin- \\
\hline & \\
\hline
\end{tabular}


Extended Data Table 4 | Gene Expression cohort description

$\begin{array}{llll}\text { Cohort } & \text { Analysis } & \text { Key } & \\ \text { GSE19491 } & \text { atb v hlt } & \text { atb } & \text { Active TB } \\ \text { GSE19491 } & \text { atb v ltb } & \text { Itb } & \text { Latent TB } \\ \text { GSE19491 } & \text { Itb v hlt } & \text { eot } & \text { End of Treatment } \\ \text { GSE28623 } & \text { atb v hlt } & \text { hlt } & \\ \text { GSE28623 } & \text { atb v ltb } & \\ \text { GSE28623 } & \text { Itb v hlt } & \\ \text { GSE29536 } & \text { atb v hlt } & \\ \text { GSE31348 } & \text { atb v eot } & \\ \text { GSE34608 } & \text { atb v hlt } & \\ \text { GSE36238 } & \text { atb v eot } & \\ \text { GSE37250 } & \text { atb v ltb } & \\ \text { GSE39939 } & \text { atb v ltb } & \\ \text { GSE39940 } & \text { atb v ltb } & \\ \text { GSE41055 } & \text { atb v hlt } & \\ \text { GSE41055 } & \text { atb v ltb } & \\ \text { GSE41055 } & \text { Itb v hlt } & \\ \text { GSE42834 } & \text { atb v hlt } & \\ \text { GSE54992 } & \text { atb v eot } & \\ \text { GSE56153 } & \text { atb v eot } & \\ \text { GSE62147 } & \text { atb v eot } & \\ \text { GSE62525 } & \text { atb v hlt } & \\ \text { GSE62525 } & \text { atb v ltb } & \\ \text { GSE62525 } & \text { Itb v hlt } & \\ \text { GSE73408 } & \text { atb v ltb } & \\ \text { GSE74092 } & \text { atb v hlt } & \\ \text { GSE74092 } & \text { atb v ltb } & \\ \text { GSE74092 } & \text { Itb v hlt } & \\ \text { GSE81746 } & \text { atb v hlt } & \\ \text { GSE83456 } & \text { atb v hlt } & \\ \text { GSE83892 } & \text { atb v hlt } & \\ \text { GSE84076 } & \text { atb v eot } & \\ \text { GSE101705 } \text { atb v ltb } & \\ \text { GSE107731 atb v hlt } & \\ \text { GSE119143 atb v hlt } & \\ \text { GSE40553S atb v eot } & \\ \text { GSE40553U atb v eot } & \\ \text { ACS } & \text { ACS } & \\ \text { Cliff } & \text { atb v eot } & \\ \text { CRTC } & \text { CRTC } & \end{array}$


Extended Data Table 5 | Metalntegrator analysis of active TB and healthy controls

\begin{tabular}{|c|c|c|c|c|c|c|c|c|c|c|c|c|c|c|}
\hline gene & effectsize & effectSizeStandardError & effectSizePval & effectSizeFDR & tausquared & numStudies & cochranesQ & heterogeneityPval & fisherStatUp & fisherPvalUp & fisherFDRUp & fisherStatDown & fisherPvalDown & fisherFDRDown \\
\hline CD8A & -0.828553701 & 0.104622781 & $2.39 \mathrm{E}-15$ & $9.30 \mathrm{E}-14$ & 0.050389462 & 12 & 24.81600649 & 0.009698998 & 3.12536185 & 0.999999895 & \begin{tabular}{|l|l|}
50.999999944 \\
\end{tabular} & 196.9139048 & $4.13 \mathrm{E}-29$ & $4.03 \mathrm{E}-28$ \\
\hline CD3E & -1.381909016 & 0.198069911 & $3.02 \mathrm{E}-12$ & $5.89 \mathrm{E}-11$ & 0.280537326 & 12 & \begin{tabular}{|l|}
45.14028777 \\
\end{tabular} & $4.58 \mathrm{E}-06$ & 2.942933927 & 0.999999944 & 0.999999944 & 340.8219093 & $9.24 \mathrm{E}-58$ & $3.60 \mathrm{E}-56$ \\
\hline $\begin{array}{l}\text { PDCD1LG2 } \\
\end{array}$ & 1.044332929 & 0.157800311 & $3.64 \mathrm{E}-11$ & $4.73 E-10$ & 0.146295916 & 12 & 28.18500549 & 0.003032616 & 233.356776 & $3.20 \mathrm{E}-36$ & $2.50 \mathrm{E}-35$ & 2.296953309 & 0.999999996 & \\
\hline ITGAM & 1.076483238 & 0.199472155 & $6.79 \mathrm{E}-08$ & $6.62 \mathrm{E}-07$ & 0.279844153 & 12 & 42.9066826 & $1.13 \mathrm{E}-05$ & 209.495939 & $1.50 \mathrm{E}-31$ & $9.76 \mathrm{E}-31$ & 1.536194882 & 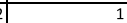 & 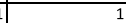 \\
\hline CD36 & 0.859949312 & 161090208 & $9.38 \mathrm{E}-08$ & $7.32 \mathrm{E}-07$ & 0.204122494 & 12 & 74.47020154 & $1.71 \mathrm{E}-11$ & 265.6288021 & $1.29 \mathrm{E}-42$ & $2.52 \mathrm{E}-41$ & 10.85051706 & 0.990038697 & \\
\hline ICOS & -0.928726858 & 0.193702989 & $1.63 \mathrm{E}-06$ & $1.06 E-05$ & 0.259041834 & 12 & \begin{tabular}{|l|l|}
39.74938398 \\
\end{tabular} & $3.95 \mathrm{E}-05$ & 7.288554416 & \begin{tabular}{|l|l|} 
& 0.999587926 \\
\end{tabular} & \begin{tabular}{|l|l|}
5.999999944 \\
\end{tabular} & 243.1268341 & $3.79 \mathrm{E}-38$ & $4.92 \mathrm{E}-37$ \\
\hline CD163 & 0.783635553 & 0.174681187 & $7.25 \mathrm{E}-06$ & 4.04E-05 & 0.214685686 & 12 & 69.33669906 & $1.63 \mathrm{E}-10$ & 187.8349229 & $2.32 \mathrm{E}-27$ & $1.29 \mathrm{E}-26$ & 3.765164186 & 0.999999265 & \\
\hline \begin{tabular}{|l|} 
FCGR3A \\
\end{tabular} & 0.487532628 & 0.110433085 & $1.01 \mathrm{E}-05$ & $4.59 \mathrm{E}-05$ & 0.046595953 & 11 & \begin{tabular}{|l|}
19.06669791 \\
\end{tabular} & 0.03942365 & 112.9571255 & $3.26 \mathrm{E}-14$ & $1.06 \mathrm{E}-13$ & 5.2625988 & 0.99990383 & \\
\hline ACTA2 & 0.920528437 & 0.209849427 & $1.15 \mathrm{E}-05$ & 4.59E-05 & 0.348243226 & 12 & 50.74051454 & 4.60E-07 & \begin{tabular}{|l|l|}
247.99664 \\
\end{tabular} & $4.26 \mathrm{E}-39$ & $4.16 \mathrm{E}-38$ & 6.561176703 & 0.999838028 & \\
\hline CD4 & -0.692535265 & 3039365 & $18 \mathrm{E}-05$ & $59 \mathrm{E}-05$ & 0.158641546 & 12 & 31.16042266 & 001038737 & 8.790840639 & 998009236 & 999999944 & 139.2649438 & $3.18 \mathrm{E}-18$ & $2.07 \mathrm{E}-17$ \\
\hline \begin{tabular}{|l|} 
MPO \\
\end{tabular} & 0.695404051 & 0.1660425 & $2.81 \mathrm{E}-05$ & $9.97 E-05$ & \begin{tabular}{|l|l|}
0.168766155 \\
\end{tabular} & 12 & \begin{tabular}{|l|}
31.08074596 \\
\end{tabular} & 0.001069484 & 153.4568664 & $7.53 \mathrm{E}-21$ & $3.67 \mathrm{E}-20$ & 7.379232178 & 0.999541125 & 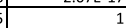 \\
\hline \begin{tabular}{|l|} 
IDO1 \\
\end{tabular} & 0.774866873 & 0.212420832 & 000264504 & 000859639 & \begin{tabular}{|l|l|}
0.262704777 \\
\end{tabular} & 10 & \begin{tabular}{|l|}
31.84657789 \\
\end{tabular} & 0.00021166 & \begin{tabular}{|l|}
138.7573995 \\
\end{tabular} & $8.71 \mathrm{E}-20$ & $\begin{array}{l}3.77 E-19 \\
\end{array}$ & 5.783079405 & 0.999160323 & \\
\hline $\begin{array}{ll}\text { CD14 } \\
\end{array}$ & 0.981011221 & 0.274968029 & 0.00036009 & 0.001080269 & 0.654378571 & 12 & \begin{tabular}{|l|}
129.6267266 \\
\end{tabular} & 0 & 257.9593359 & $4.34 \mathrm{E}-41$ & $5.65 \mathrm{E}-40$ & 12.12134564 & 0.97850626 & \\
\hline TNFRSF18 & -0.500 & 6968 & 029862 & 0.005654615 & 0.17 & 12 & 45.93125783 & $3.33 \mathrm{E}-06$ & 12.66440441 & .971335513 & 0.999999944 & 2758829 & $3.73 \mathrm{E}-12$ & $1.82 \mathrm{E}-11$ \\
\hline CD274 & 1.27655224 & 2805 & 0.002431499 & 0.006321897 & 2.092833485 & 13 & 84236 & & 64537 & $4.20 \mathrm{E}-81$ & $1.64 \mathrm{E}-79$ & 6286 & $8.89 \mathrm{E}-12$ & $3.85 \mathrm{E}-11$ \\
\hline \begin{tabular}{|l|} 
IL10 \\
\end{tabular} & 0.30632555 & 0.115112571 & 0.007788685 & 0.01898492 & 0.079745264 & 12 & \begin{tabular}{|l|}
40.61123656 \\
\end{tabular} & $2.81 \mathrm{E}-05$ & \begin{tabular}{|l|l|}
90.91544911 \\
\end{tabular} & $1.02 \mathrm{E}-09$ & $2.83 \mathrm{E}-09$ & 22.40562025 & 0.555070627 & 0.746474291 \\
\hline MS4A1 & -0.509330842 & 0216 & 0.009881961 & 0.022670382 & 0.365156472 & 13 & \begin{tabular}{|l|}
118.1340154 \\
\end{tabular} & 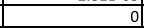 & \begin{tabular}{|l|}
57.67296161 \\
\end{tabular} & 000341825 & 0.00074062 & 272.2138207 & $7.17 \mathrm{E}-43$ & $1.40 \mathrm{E}-41$ \\
\hline LAG3 & -0.386130023 & 167998956 & 0.02153862 & $\begin{array}{l}0.04666701 \\
\end{array}$ & 0.18223159 & 12 & \begin{tabular}{|l|}
33.63106147 \\
\end{tabular} & 0.000415497 & 14.48675115 & \begin{tabular}{|l|l|} 
& 0.934882201 \\
\end{tabular} & \begin{tabular}{|l|l|} 
& 0.999999944 \\
\end{tabular} & 88.07223987 & $3.00 \mathrm{E}-09$ & $1.06 \mathrm{E}-08$ \\
\hline PTPRC & 57846 & 5934411 & 0.041108988 & 082908725 & & 12 & 202.430536 & & 116 & $3.66 \mathrm{E}-14$ & $1.10 \mathrm{E}-13$ & & 000105817 & 00294775 \\
\hline \begin{tabular}{|l|} 
MRC1 \\
\end{tabular} & 0.23122355 & 0.114230994 & 0.042952125 & 0.082908725 & 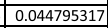 & & 16.04445161 & 041748274 & \begin{tabular}{|l|l|}
52.89490043 \\
\end{tabular} & $2.72 \mathrm{E}-05$ & $6.64 \mathrm{E}-05$ & 00224 & .735845132 & 0.956598672 \\
\hline PDCD1 & 772466 & 66385 & 0.04464316 & 0.082908725 & 0.310588128 & 11 & 355691 & 4.19E-07 & \begin{tabular}{|l|}
16.01436202 \\
\end{tabular} & 0.815172355 & \begin{tabular}{|l|l|}
0.963385511 \\
\end{tabular} & 7348 & $1.03 \mathrm{E}-12$ & $5.73 \mathrm{E}-12$ \\
\hline NCAM1 & -0.187047872 & 994483367 & 0.04773826 & 0.084626916 & 0.052456397 & 12 & 33.48110851 & 000439532 & 31.32922614 & \begin{tabular}{|l|l|} 
& 0.144515061 \\
\end{tabular} & 0.194347841 & 84.27278616 & $1.25 \mathrm{E}-08$ & $4.07 \mathrm{E}-08$ \\
\hline ITGAX & 0.314735494 & 91326066 & 0.099965377 & 0.169506508 & 0.302992435 & 12 & \begin{tabular}{|l|}
105.0064573 \\
\end{tabular} & 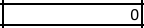 & 146.4917225 & $1.48 \mathrm{E}-19$ & $5.78 \mathrm{E}-19$ & 50.5031268 & 0.001221365 & 0.002801955 \\
\hline CD68 & & & & 0.182667143 & & 13 & & 0 & & 2.62E-18 & $9.28 \mathrm{E}-18$ & & .053086371 & 0.098588974 \\
\hline \begin{tabular}{|l|} 
CTLA4 \\
\end{tabular} & -0.294867517 & 88779 & 0.117475857 & 0.183262337 & 45396 & 13 & \begin{tabular}{|l|}
107.8652589 \\
\end{tabular} & 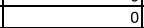 & 41.48339134 & 0.027728545 & 0.041592818 & 152.3602194 & $7.77 \mathrm{E}-20$ & $6.06 \mathrm{E}-19$ \\
\hline CMA1 & & & 557487 & 3623 & 0.05 & 12 & 17.4 & 0.096143803 & 5957 & 0.02 & & & 0.484791945 & 0.700255031 \\
\hline TGFB1 & -0.462048879 & 4421165 & 0.267183903 & 0.385932304 & \begin{tabular}{|l|l|}
1.850128268 \\
\end{tabular} & 12 & 391.363167 & & 45.48093334 & 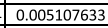 & 0.009054441 & 84.05633166 & $1.36 \mathrm{E}-08$ & 4.07E-08 \\
\hline \begin{tabular}{|l|} 
NOS2 \\
\end{tabular} & 0.118174792 & 329204 & 0.288467457 & 401793958 & 0.053310225 & 10 & 20.82502366 & 0.013450411 & 43.88373597 & 0.001559817 & 0.002896803 & 38.05223588 & 008726349 & .017911979 \\
\hline \begin{tabular}{|l|l|} 
MKI67 \\
\end{tabular} & 247 & 0695 & 0.310320146 & 17092 & & 12 & \begin{tabular}{|l|}
8.70 \\
\end{tabular} & 0.648840545 & 33.6 & \begin{tabular}{|l|l|}
0.091594441 \\
\end{tabular} & \begin{tabular}{|l|l|} 
& 0.127577972 \\
\end{tabular} & 3616 & 9199779 & 0.723171121 \\
\hline $\begin{array}{ll}\text { CD209 } \\
\end{array}$ & 0.097565064 & 5576559 & 0.496799965 & 645839954 & 0.13423952 & 12 & \begin{tabular}{|l|}
31.90395772 \\
\end{tabular} & 0.000790266 & \begin{tabular}{|l|l|}
57.66973162 \\
\end{tabular} & 0.000135214 & 0.000310196 & 33.79607962 & 38358992 & 0.149826116 \\
\hline ITGAE & -0.122975443 & 2668 & 0.526049021 & 0.661803607 & 0.266397944 & 12 & \begin{tabular}{|l|}
43.73135699 \\
\end{tabular} & $8.10 \mathrm{E}-06$ & 41.13889116 & \begin{tabular}{|l|l|l|l|} 
\\
\end{tabular} & 0.026132977 & 7239 & 77703 & 0.001055898 \\
\hline HAVCR & 0.1069 & 632 & 0.627504518 & 764771131 & \begin{tabular}{|l} 
\\
0.410797002 \\
\end{tabular} & 12 & \begin{tabular}{|l|l|}
77.98623747 \\
\end{tabular} & $3.61 \mathrm{E}-12$ & 89.9 & $1.49 \mathrm{E}-09$ & $3.87 \mathrm{E}-09$ & & 000433189 & .001055898 \\
\hline PECAN & 341355 & 2209 & 0.671545881 & 0.7936 & 0.471477769 & 12 & \begin{tabular}{|l|}
70.06389603 \\
\end{tabular} & $\begin{array}{l}1.19 \mathrm{E}-10 \\
\end{array}$ & 38.33624397 & 0.032019106 & 0.04624982 & 94.25844327 & $2.81 \mathrm{E}-10$ & $1.10 \mathrm{E}-09$ \\
\hline VIM & 0.049650097 & 162760816 & 0.760328493 & 0.847240404 & \begin{tabular}{|l|l|} 
\\
\end{tabular} & 12 & \begin{tabular}{|l|}
53.61638631 \\
\end{tabular} & $1.39 \mathrm{E}-07$ & \begin{tabular}{|l|}
51.49080928 \\
\end{tabular} & \begin{tabular}{|l|l|} 
& 0.000911202 \\
\end{tabular} & 0.001776844 & 40.22567594 & 0.020223404 & 0.039435639 \\
\hline TPSAB & & & 7244 & 40404 & 0.01 & & \begin{tabular}{|l|}
10.59599818 \\
\end{tabular} & 0.225657497 & 19.1224661 & 0.384318755 & & 20.88182567 & 0.285416969 & 0.428125454 \\
\hline \begin{tabular}{|l|} 
COL1AA1 \\
\end{tabular} & 0.047688602 & 0.172394404 & 0.782068066 & 0.847240404 & 0.230123208 & 12 & \begin{tabular}{|l|l|}
70.77368141 \\
\end{tabular} & $8.71 \mathrm{E}-11$ & 41.42530877 & 0.01495297 & 0.025355036 & 33.02098971 & 0.103631369 & 0.168400974 \\
\hline IFNG & & & & 182 & & 1 & 172. & & 6824 & 0.000 & & & & \\
\hline $\mathrm{CDH} 1$ & -0.01 & 344 & & 0.8 & 0.016967086 & 12 & 15.4 & 459 & 9729 & 0.462 & 083 & 8813 & 0.059159563 & 0.1048 \\
\hline \begin{tabular}{|l|l|} 
FOXP3 \\
\end{tabular} & -0.001586775 & 0.080386328 & 0.984251289 & 0.984251289 & 0 & 12 & 10.84236152 & 0.456562797 & 26.42461843 & 0.331977444 & \begin{tabular}{|l}
0.431570677 \\
\end{tabular} & 27.9828023 & 0.260765979 & 0.406794927 \\
\hline
\end{tabular}




\section{Reporting Summary}

Nature Research wishes to improve the reproducibility of the work that we publish. This form provides structure for consistency and transparency in reporting. For further information on Nature Research policies, see our Editorial Policies and the Editorial Policy Checklist.

\section{Statistics}

For all statistical analyses, confirm that the following items are present in the figure legend, table legend, main text, or Methods section.

n/a Confirmed

\ The exact sample size $(n)$ for each experimental group/condition, given as a discrete number and unit of measurement

\ A statement on whether measurements were taken from distinct samples or whether the same sample was measured repeatedly

The statistical test(s) used AND whether they are one- or two-sided

Only common tests should be described solely by name; describe more complex techniques in the Methods section.

\ A description of all covariates tested

$\square$ A description of any assumptions or corrections, such as tests of normality and adjustment for multiple comparisons

$\checkmark$ A full description of the statistical parameters including central tendency (e.g. means) or other basic estimates (e.g. regression coefficient)

AND variation (e.g. standard deviation) or associated estimates of uncertainty (e.g. confidence intervals)

For null hypothesis testing, the test statistic (e.g. $F, t, r$ ) with confidence intervals, effect sizes, degrees of freedom and $P$ value noted

Give $P$ values as exact values whenever suitable.

Х $\square$ For Bayesian analysis, information on the choice of priors and Markov chain Monte Carlo settings

$\square$ \ For hierarchical and complex designs, identification of the appropriate level for tests and full reporting of outcomes

$\square \bigotimes$ Estimates of effect sizes (e.g. Cohen's $d$, Pearson's $r$ ), indicating how they were calculated

\section{Our web collection on statistics for biologists contains articles on many of the points above.}

\section{Software and code}

Policy information about availability of computer code

Data collection MIBI-TOF data collection software (MinisIMS version 5.5.4.0)

Data analysis Matlab 2016a, Matlab 2019b, R 3.6.2 (all packages are listed in a document at the Github link listed below), Python3.6, QuPath 0.2.3. All custom code available at: https://github.com/angelolab/publications/2022-McCaffrey_etal_HumanTB

For manuscripts utilizing custom algorithms or software that are central to the research but not yet described in published literature, software must be made available to editors and

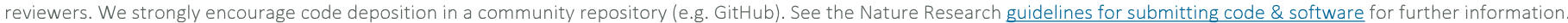

Data

Policy information about availability of data

All manuscripts must include a data availability statement. This statement should provide the following information, where applicable:

- Accession codes, unique identifiers, or web links for publicly available datasets

- A list of figures that have associated raw data

- A description of any restrictions on data availability

All images and annotated single cell data can be accessed through Mendeley's data repository at the following link: DOI: 10.17632/dr5fkgtrb6. The GEO accession numbers for all transcriptomic data used in our meta-analysis can be found in Extended Data Table 4. 
Please select the one below that is the best fit for your research. If you are not sure, read the appropriate sections before making your selection.

$\bigotimes$ Life sciences $\quad \square$ Behavioural \& social sciences $\quad \square$ Ecological, evolutionary \& environmental sciences

For a reference copy of the document with all sections, see nature.com/documents/nr-reporting-summary-flat.pdf

\section{Life sciences study design}

All studies must disclose on these points even when the disclosure is negative.

Sample size Sample size was not statistically determined. Cohort sample size was based on specimen availability and histological selection criteria to screen samples available from Stanford, AHRI, and University of Texas Health Science Center tissue repositories.

Data exclusions No data excluded

Replication All cohort specimens were prepared and analyzed alongside control tissue specimens to validate the technical performance of all reagents, instrument settings, and analysis parameters used in the study. Antibody reagents were extensively validated on control tissues with immunohistochemistry and MIBI-TOF analysis. Data collection was performed on all specimens once.

Randomization This is not relevant because there were no separate experimental groups in this study. The cohort was a retrospective analysis of archival clinical tissue specimens. Controls were used for technical validation of antibodies, but not for comparative analysis. All samples were prepared together and imaged in a random order.

Blinding The study did not require blinding as it was a retrospective analysis of archival clinical tissue specimens. That said, data collection and analysis were not performed blind to the type of specimen.

\section{Reporting for specific materials, systems and methods}

We require information from authors about some types of materials, experimental systems and methods used in many studies. Here, indicate whether each material, system or method listed is relevant to your study. If you are not sure if a list item applies to your research, read the appropriate section before selecting a response.

\begin{tabular}{|c|c|c|c|}
\hline \multicolumn{2}{|c|}{ Materials \& experimental systems } & \multicolumn{2}{|c|}{ Methods } \\
\hline $\mathrm{n} / \mathrm{a}$ & Involved in the study & $\mathrm{n} / \mathrm{a}$ & Involved in the study \\
\hline L & $\bigotimes$ Antibodies & Х & $\square$ ChIP-seq \\
\hline - & $\bigotimes$ Eukaryotic cell lines & Х & $\square$ Flow cytometry \\
\hline$\bigotimes$ & $\square$ Palaeontology and archaeology & Х & $\square$ MRI-based neuroimaging \\
\hline$\bigotimes$ & $\square$ Animals and other organisms & & \\
\hline L & $\bigotimes$ Human research participants & & \\
\hline$\bigotimes$ & $\square$ Clinical data & & \\
\hline$\bigotimes$ & $\square$ Dual use research of concern & & \\
\hline
\end{tabular}

\section{Antibodies}

Antibodies used Study used 39 primary antibodies. See extended data table 2 for all requested information of each reagent including the precise working concentration used (called "Titer").

Validation

All reagents were validated in-house with chromogenic immunohistochemistry on FFPE human control tissues. Following this stage of validation all antibodies were metal-labeled and further tested with MIBI-TOF. Imaging data for all antibodies in control tissues (spleen, tonsil, placenta) can be found in our data repository.

\section{Eukaryotic cell lines}

Policy information about cell lines

Cell line source(s)

Commercial control slides containing FFPE cultured cell pellets of human HeLa cells from Advanced Cell Diagnostic (ACD)

Authentication

NA

Mycoplasma contamination

NA 


\section{Human research participants}

Policy information about studies involving human research participants

Population characteristics

Recruitment

Ethics oversight
Study did not directly involve human participants, but used archival clinical specimens from individuals with active Mycobacterium tuberculosis infection. Individuals were treated in the U.S. $(n=12)$ or South Africa $(n=3)$. Study included both male and female individuals with reported ages of 19-88 years.

Study was done on archival clinical specimens from tissue repositories. No recruitment was done.

All human samples were acquired in accordance with Institutional Review Board (IRB) protocol \#46586: "Generation of an Immune Atlas of Human Tuberculosis Granulomas with Multiplexed Ion Beam Imaging."

Note that full information on the approval of the study protocol must also be provided in the manuscript. 